\title{
Success Factors of Process Digitalization Projects - Insights from an Exploratory Study
}

Original Research

\begin{abstract}
Purpose - Digitalization substantially impacts organizations, which increasingly use digital technologies to improve and innovate their business processes. While there are methods and tools for identifying process digitalization ideas and related projects (PDPs), guidance on the successful implementation of PDPs is missing. Hence, we set out to explore PDP success factors.

Design/methodology/approach - In an exploratory approach, we conducted a structured literature review to extract candidate PDP success factors from the literature on business process management, project management, and digitalization. After that, we validated, refined, and extended these intermediate results through interviews with 21 members of diverse PDP teams. Finally, we proposed the PDP Success Model by linking the candidate success factors with relevant success criteria.

Findings - The PDP Success Model covers 38 PDP success factor candidates, whereof 28 are already backed by the literature and ten have emerged during the interviews. Furthermore, the success factors are structured according to seven categories from the literature covering a broad range of socio-technical topics (i.e., strategy, structure, culture, people, process, project, and technology) as well as equipped with preliminary success rationales.

Originality - Our work is the first to systematically explore PDP success factors. The PDP Success Model shows that PDPs require a unique set of success factors, which combine established and hitherto underrepresented knowledge. It extends the knowledge on business process management and serves as foundation for future (confirmatory) research on business process digitalization and the successful implementation of PDPs.
\end{abstract}

Keywords - Business Process Management; Digitalization; Success Factors; Literature Review; Exploratory Interviews

Article Type - Research paper 


\section{Introduction}

Digitalization is driven by the fast emergence and adoption of digital technologies (DTs), changing societal conventions and organizational routines (Beverungen et al., 2020). DTs range from established technologies (e.g., social, mobile, analytic, and cloud) (Fitzgerald et al., 2014) to emerging ones (e.g., distributed ledger, artificial intelligence, extended reality, and quantum computing) (Daugherty, 2020; Gartner, 2020). Accordingly, digitalization entails a hyper-connected environment for organizations (Beverungen et al., 2020), which is characterized by the access to new data sources, the fusion of the digital and the physical world, pervasive connectivity, and interactions among individuals, organizations, and real-world objects (Benbya et al., 2020).

Although digitalization brings about manifold opportunities, organizations struggle with deriving value from DTs (Davenport and Westerman, 2018), as they do not fully understand how to use DTs (Denner et al., 2018). Apart from the DT-enabled transformation of products into smart things (Beverungen et al., 2019; Huber et al., 2019), DTs enable organizations to improve and innovate business processes (Mendling et al., 2020). For example, DTs support advanced process automation, adaptive process execution, and process data analytics (Kerpedzhiev et al., 2020). To capitalize on the opportunities of digitalization, organizations must embed DTs into existing or novel processes (Denner et al., 2018), which commonly happens through projects (Lehnert et al., 2016; Kerzner, 2013). In our study, we refer to projects that leverage DTs for improving business processes in terms of effectiveness and efficiency as process digitalization projects (PDPs). While the literature contains methods and tools assisting practitioners in the identification of process digitalization ideas and projects (Denner et al., 2018; Rosemann, 2020), guidance on the successful implementation of PDPs is missing. This circumstance presents organizations with challenges, as PDP failure may entail sunk costs or jeopardize competitiveness (McLean and Antony, 2014).

In the literature on business process management (BPM), project management (PM), and digitalization, which are relevant reference disciplines when investigating PDP success, antecedents of successful BPM and digital transformation initiatives as well as of process improvement and digitalization projects have already been investigated. We refer to such antecedents as success factors (SFs) (Bullen and Rockart, 1981). For example, Trkman (2010), Rosemann and Vom Brocke (2015), and Castro et al. (2020) proposed SFs for BPM on the enterprise level, whereas McLean and Antony (2014) as well as Al-Mashari and Zairi (1999) studied failure factors on the project level. Some studies also focused on SFs and pitfalls related to specific activities of process change such as process modeling (Bandara et al., 2005; Rosemann, 2006). In the PM domain, McLeod et al. (2012), for example, 
investigated how project stakeholders perceive project success. As opposed to BPM and PM, research on successful digitalization projects and digital transformation initiatives is still emergent. While Gimpel et al. (2018) offer a framework of action fields for successful digital transformation of incumbent firms, Soluk and Kammerlander (2021) present barriers and enablers for the digital transformation of family-owned Mittelstand firms. These studies demonstrate that there are isolated pockets of understanding. However, an integrated and up-to-date view on factors that drive PDP success yet needs to be developed. Hence, our research question is as follows: Which factors drive PDP success?

To answer this question, we followed an exploratory approach. First, we extracted candidate SFs from the BPM, PM, and digitalization literature via a structured literature review. This review resulted in a comprehensive ex-ante list, which included 30 candidate SFs. With Kerpedzhiev et al. (2020) arguing that digitalization questions fundamental BPM assumptions, SFs retrieved from the literature most likely do not fully account for the peculiarities of PDPs. Owing to the fast-moving nature of digitalization, many first-hand experiences yet need to be documented academically. Therefore, as a second step, we conducted semi-structured interviews with 21 participants of PDPs performed in German manufacturing companies. This step resulted in a validated, refined, and extended ex-post list of candidate PDP SFs, which includes 38 elements, whereof 28 are backed by the literature and ten emerged during the interviews. Our key contribution is the PDP Success Model, which links the candidate SFs with PDP success criteria.

The remainder of this study is structured as follows: In Section 2, we provide relevant background on digitalization, BPM, and PM, before presenting our research design in Section 3. In Sections 4 and 5, we present our findings of the literature review, the interviews, and the PDP Success Model. In Section 6, we discuss implications, point to limitations, and sketch ideas for future research.

\section{Background}

\subsection{Digitalization and Digital Technologies}

Digitalization is a socio-economic phenomenon. Rather than translating information into a digital format (digitization) (Legner et al., 2017), it enables novel value propositions by embedding DTs in products (Beverungen et al., 2019; Huber et al., 2019) and processes (Kerpedzhiev et al., 2020). Digitalization has evolved into a key topic of information systems (IS) research with scientists making huge effort to understand the phenomenon and its 
effects (Majchrzak et al., 2016; Baskerville et al., 2020). Capitalizing on new data sources, the pervasiveness of computing capabilities increases connectedness (Benbya et al., 2020) to the extent that the fusion of the physical and digital world is just one facet of the digital society (Matt et al., 2015). Despite attempts to identify relevant action fields of digital transformation (Gimpel et al., 2018; Wessel et al., 2021), knowledge is in its infancy (Benbya et al., 2020; Vial, 2019). Hence, it presents new opportunities and challenges (Loebbecke and Picot, 2015).

Digitalization is driven by DTs (Daugherty, 2020; Berger et al., 2018). While the general term technology is wellunderstood (Arthur, 2009), the term DT is used as an umbrella term for information technology in the context of digitalization (Denner et al., 2018). Hence, one cannot draw clear lines between information technology, information systems, and DTs. Yoo et al. (2010) proposed fundamental characteristics to differentiate DTs from other types of technology: (1) homogenization of data, (2) re-programmability, and (3) self-referential nature. Moreover, DTs have been characterized as embedded, connected, editable, communicable, identifiable, and associable in line with their foundation in symbol-based computation (Benbya et al., 2020). Coining the term ontological reversal, Baskerville et al. (2020) even proposed that the classical view of IS as representations of physical reality will become obsolete, as DTs create and shape physical reality. In practice, the Gartner Hype Cycle of Emerging Technologies is a prominent tool in the DT context (Gartner, 2020). Its 2020 edition includes DTs such as affective computing, distributed ledger, and smart advisors to generative artificial intelligence, authenticated provenance, and digital twins. Another popular practice-oriented classification of DTs follows the SMAC acronym (i.e., social, mobile, analytics, and cloud) (Fitzgerald et al., 2014), while emergent DTs are subsumed under the DARQ acronym (i.e., distributed ledger, artificial intelligence, extended reality, and quantum computing) (Daugherty, 2020).

\subsection{Business Process Management and Improvement}

BPM is the science and practice of overseeing business processes to ensure consistent outcomes and that improvement opportunities are seized (Dumas et al., 2018). BPM drives corporate success through efficient and effective business processes, both of which are relevant success criteria of PDPs (Schmiedel et al., 2020; Morana et al., 2019). Operationally speaking, PDPs must advance cost, flexibility, quality, time, and/or customer satisfaction (Reijers and Limam Mansar, 2005; Kreuzer et al., 2020). Process digitalization increases efficiency and quality. The dependence of processes on engineered devices also gives rise to concerns about their safety and integrity (Khan et al., 2021). Emergent technologies play an important role in increasing process safety (Ahmed, 2021; Sajid et al., 2021), for example, through DT-assisted process fault prognosis (Arunthavanathan et al., 2021; 
Adumene et al., 2021). From a lifecycle perspective, BPM includes activities such as process identification, discovery, analysis, implementation, execution, monitoring, controlling, and improvement (Recker and Mendling, 2016; Kerpedzhiev et al., 2020). Combining knowledge from the management sciences and information technology (van der Aalst, 2013), BPM enables organizations to leverage DTs for process improvement and innovation (Denner et al., 2018; Mendling et al., 2020).

Among the activities from the BPM lifecycle, process improvement and innovation are considered the most valueadding ones (Denner et al., 2018; Rosemann and Vom Brocke, 2015). An important distinction in this context is that between continuous business process improvement (BPI) and business process reengineering (BPR) (Trkman, 2010). Another distinction is that between exploitative and explorative process change (Grisold et al., 2019). Exploitative process change applies methods from BPI and BPR to reactively fix problems of existing processes with the redesigned processes featuring either the same or an enhanced value proposition. Explorative process change seizes opportunities to implement so far non-existent value propositions in new or existing processes. In the latter case, reengineered processes feature either the same or an enhanced value proposition. Exploitative and explorative process change typically make use of DTs. Our PDP definition presented in the introduction covers DT-enabled exploitative process change as well as DT-enabled explorative BPM that seizes opportunities to reengineer existing processes. We restricted our definition that way, as explorative process change with a focus on the creation of new processes is hardly supported by appropriate methods so far (Vom Brocke et al., 2020). Hence, there is no related literature on PDP success that could have been analyzed.

\subsection{Project and Project Portfolio Management}

Process change usually happens through projects (Lehnert et al., 2016), i.e., punctuated efforts of interrelated tasks undertaken to achieve predefined objectives within a concrete timeframe (Archibald, 2003). PM involves activities such as planning, monitoring, execution, and control as well as the motivation of project participants within defined time, cost, and quality (Clarke, 2016; Kerzner, 2013; Pellegrinelli et al., 2015). Analogous to processes, project success is commonly assessed via effectiveness and efficiency (Drucker, 2007; Beer et al., 2013). While PM ensures the success of single projects (Cooke-Davies, 2002), project portfolio management (PPM) focuses on multiple interdependent projects (Martinsuo et al., 2014) The portfolio perspective is key, as organizations typically implement multiple interdependent projects at the same time with resources being scarce for simultaneous project implementation (de Reyck et al., 2005). This applies to projects in general and to PDPs. 


\section{Research Design}

Our research design, which details the Figure 1, included the following steps: (1) structured database search, (2) code extraction from the literature and building of the ex-ante list of candidate SFs, (3) semi-structured expert interviews, (4) code extraction from the interviews and building of the ex-post list of candidate SFs. Finally, we (5) compiled the PDP Success Model. The research process is summarized in Table 1. Note that, in line with the exploratory nature of our work, all SFs included in the PDP Success Model are to be treated as candidates.

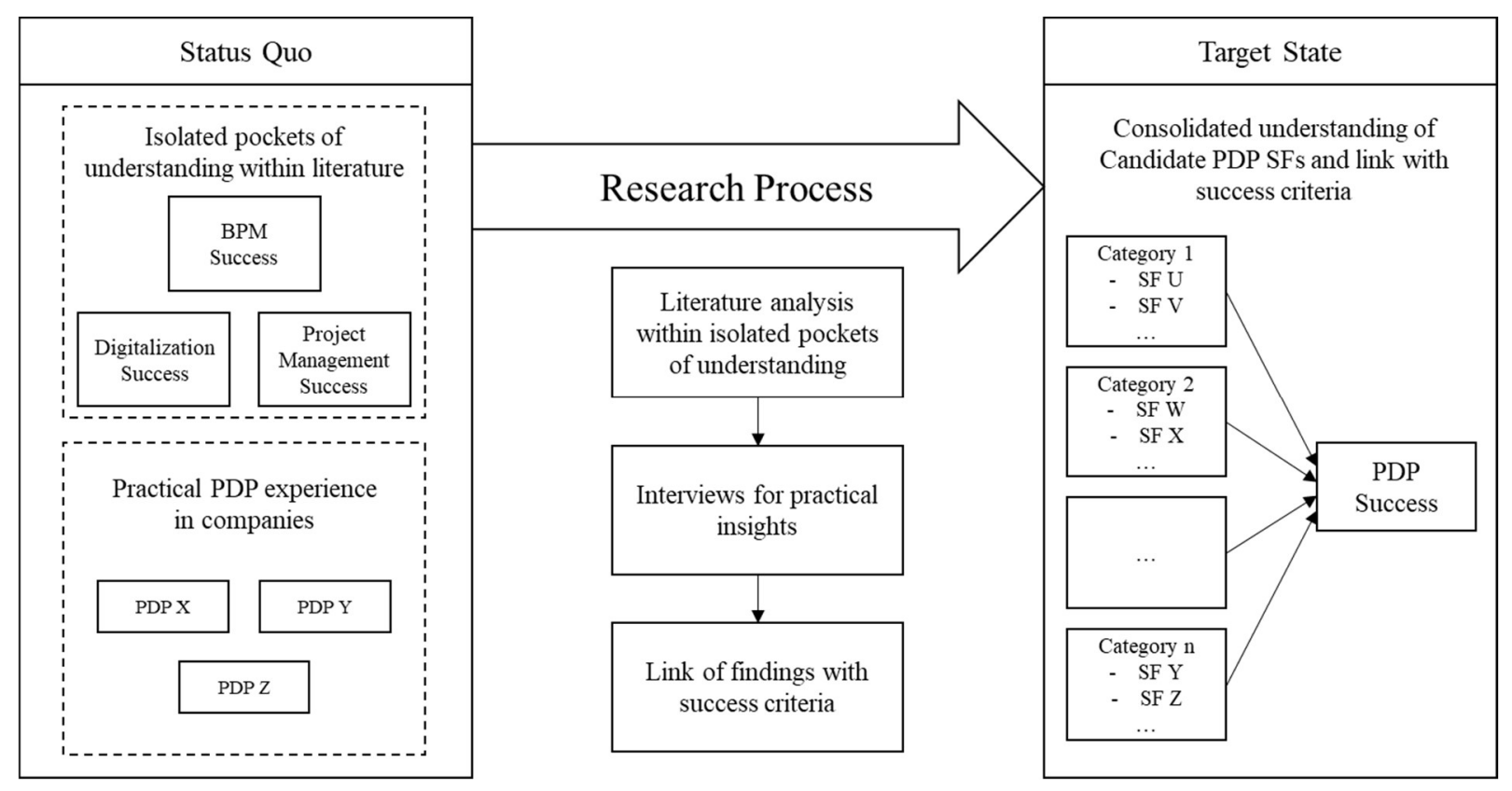

Fig. 1 Research process of the paper

Table 1 Research Design

\begin{tabular}{|c|c|c|c|}
\hline$\#$ & Step & Action & Result \\
\hline 1 & Literature review & $\begin{array}{l}\text { Database search and } \\
\text { systematic literature review }\end{array}$ & $\begin{array}{l}645 \text { identified papers, } \\
40 \text { selected papers }\end{array}$ \\
\hline 2 & $\begin{array}{l}\text { Code extraction and building of the } \\
\text { ex-ante list }\end{array}$ & Text analysis & $\begin{array}{l}1034 \text { initial SF codes, } \\
30 \text { candidate SFs, } \\
7 \text { SF categories }\end{array}$ \\
\hline 3 & Expert interviews & $\begin{array}{l}\text { Semi-structured interviews with ex- } \\
\text { perts from real-world PDPs }\end{array}$ & $\begin{array}{l}21 \text { interviewees with experts from } \\
7 \text { PDPs and } 4 \text { companies }\end{array}$ \\
\hline 4 & $\begin{array}{l}\text { Code extraction and building of the } \\
\text { ex-post list }\end{array}$ & Interview analysis & $\begin{array}{l}7 \text { additional candidate SFs, } \\
2 \text { refined candidate SFs resulting in } 3 \\
\text { new ones, } \\
9 \text { SFs without supporting empirical } \\
\text { evidence }\end{array}$ \\
\hline 5 & $\begin{array}{l}\text { Compilation of the } \\
\text { PDP Success Model }\end{array}$ & $\begin{array}{l}\text { Linking of independent and dependent } \\
\text { variables }\end{array}$ & $\begin{array}{l}38 \text { candidate } \mathrm{SFs}, \\
7 \mathrm{SF} \text { categories, } \\
2 \text { dependent variables }\end{array}$ \\
\hline
\end{tabular}


We conducted the structured database search (step 1), following the recommendations of Vom Brocke et al. (2015). We searched the databases of the AIS electronic Library, EBSCOhost, and ScienceDirect and checked the 'Senior Scholars' Basket of Journals' from the IS discipline. All selected studies included a combination of the following terms in the title, abstract, or keywords: ("Success*” OR “Fail*”) AND “Factor*” AND ("Digit*” OR "Digital Transformation" OR "Process Improvement" OR "Process Reengineering” OR "Project Management" OR “Project Portfolio Management”). The search yielded 645 studies including 110 duplicates. To identify the studies appropriate for the in-depth analysis, two co-authors independently screened all abstracts and rated the studies as appropriate, questionable, or inappropriate (Wolfswinkel et al., 2013). In the case of different ratings, all co-authors read the related studies and resolved conflicts. The research team excluded studies that did not support the aim of building an ex-ante list of candidate PDP SFs. A lack of relevance to PDPs as well as insufficient transferability served as exclusion criteria. Levi et al. (2003), for example, was part of the sample due to its mention of the search terms 'success factor' and 'digitalizability' in the abstract. However, the study is about electronic exchange in the B2B area, a circumstance that led to the exclusion of the paper. As we read the studies, we continuously augmented our literature base through forward and backward search (Webster and Watson, 2002). The 101 studies rated as appropriate for in-depth analysis were read end-to-end. During the in-depth analysis, we excluded 52 studies initially rated as appropriate due to a lack of relevance for our purposes. We further excluded eleven papers that did not fit the PDP context. In the end, 40 papers provided the foundation for the next step (see Appendix A for the complete list of the papers analyzed).

While conducting our in-depth analysis (step 2), we noted explicitly and implicitly mentioned factors. First, we extracted the factors explicitly mentioned in the literature (e.g., in tables or the text). We then screened the fulltexts, marked all passages pointing to potential success or failure factors, and compiled the results (Webster and Watson, 2002). Next, we interpreted the marked passages to extract factors implicitly indicated to contribute to success or failure (Krippendorff, 2013). Kirsch and Slaughter (2013), for example, stressed the link between maturity of software development processes and project performance. Hence, we derived 'maturity of software' as a success code and later inferred the SF candidate 'technology maturity' from this as well as from other success codes. After this, we synthesized general information about the studies, along with categories such as the authors' names, publication date, and meta-information about the study context. Overall, we extracted 1034 codes, of which 
237 related to implicitly mentioned factors (see Appendix B for the complete list of success codes). Moreover, we did not exclude results related to failure factors $(157$ codes $)$ but rephrased them to align their polarity. ${ }^{1}$

Using a protocol to code the extracted factors (Corbin and Strauss, 1990), we built the ex-ante list of candidate SFs. To measure inter-coder reliability, we used Krippendorff's (2013) Alpha. In a first step, two co-authors used open coding to build 53 candidate SFs from the 1034 codes (Corbin and Strauss, 1990). Another co-author allocated 360 randomly ordered codes to the previously established candidate SFs, thereby meeting the required sample size to validly calculate an alpha of 0.8 or higher (Krippendorff, 2013). The final inter-coder reliability scored 0.8360, representing satisfactory inter-coder reliability (Krippendorff, 2013). Between all coding steps, the coauthors discussed the candidate SFs and their definitions to align the level of abstraction and to merge similar SFs. This led to 30 candidate SFs in the ex-ante list. For each candidate, the author team developed a single-sentence definition based on related papers from the literature review. These definitions can be found in Table 3.

To obtain an unbiased assessment of inter-coder reliability, an external coder from the IS field analyzed the 1034 codes and matched them to the candidate SFs, yielding a Krippendorff's Alpha between the co-authors and the external coder of 0.7333 . As our efforts resulted in a large number of candidate SFs, the low Alpha value is not surprising, and it is common practice for researchers to use Alphas of 0.7 for hypotheses such as candidate SFs (Krippendorff, 2013). Thus, we proceeded with the developed candidate SFs. Next, selective coding helped us to develop categories for grouping the SF candidates included in the ex-ante list (Webster and Watson, 2002; Wolfswinkel et al., 2013). In total, we identified seven SF categories based on an analysis of existing frameworks from literature. More details regarding the development of these categories are presented in Section 4.1.

To validate, extend, and refine the literature-based ex-ante list of candidate SFs, we conducted semi-structured expert interviews (step 3) (Galliers and Huang, 2012; Heidt et al., 2019; Myers and Newman, 2007). As outlined justified in the introduction, this step enabled us to see "the world through the eyes of the actors doing the acting" (Greener, 2008, p. 17) and to gain access to first-hand PDP experience. Moreover, expert interviews are frequently used to explore and challenge candidate SFs (Lange et al., 2016). During the interviews, we presented the

\footnotetext{
${ }^{1}$ Hughes et al. (2017), for instance, find that poor goals and evaluation stages (code no. 786) lead to IS project failure. We concluded that sophisticated objectives and evaluation stages lead to reduced levels of failure and, thereby, greater levels of success.
} 
definitions of the candidate SFs from the ex-ante list to the experts. After 21 interviews with experts from seven PDPs conducted in four manufacturing companies, we arrived at consistent results regarding the support and refinement of candidate SFs. Therefore, we deemed no further contribution through additional interviews since theoretical saturation had been reached (Marshall et al., 2013). To obtain a valid picture of the PDPs in focus, we interviewed at least three experts per company. These experts had different roles, such as project manager, project sponsor, and as an employee with technical or functional background. The reasons for targeting these groups were that project sponsors oversee the definition of PDP goals, while project managers are responsible for operational planning and monitoring. Employees are responsible for the implementation of the PDP. On the aggregate, we sought to cover various personal and professional backgrounds, as PDPs are interdisciplinary and complex (Bhattacherjee, 2012). Moreover, a sample size of around 20 experts is common for this kind of research (Abdelkafi and Pero, 2018; Heidt et al., 2019; Reif et al., 2019). It supports establishing a trusted atmosphere with the interviewees, which in turn leads to in-depth and valid insights (Crouch and McKenzie, 2006).

To compile the interview circle, we identified experts with substantial experience in process digitalization by being actively involved in or leading PDPs (Myers and Newman, 2007) (Appendix C depicts the whole expert sample). Moreover, we decided to focus on experts working for manufacturing companies for four reasons: First, PDPs play an important role in the industrial sector. Second, our industry network has distinguished outreach in the manufacturing domain, which enabled us to cover diverse contexts, companies, and PDPs. Third, we thereby reduced the complexity of understanding sector-specific mechanisms (Davis and Hufnagel, 2007). Fourth, the PDPs typically performed in manufacturing companies fit our PDP definition quite well, covering exploitative DT-enabled process change as well as explorative DT-enabled process change with a focus on reengineering existing processes. As our focus on manufacturing companies may limit the transferability of our results, we get back to this limitation in Section 5. The PDPs in which the interviewees were involved were performed in four companies: Polymer (P), Automotive (A), Healthcare (H), and Textile (T). We selected companies that actively engaged in process digitalization and interviewed members of the PDP teams who met our selection criteria. Overall, the experts were involved in seven PDPs (P1, P2, A1, H1, H2, H3, T1). Table 2 provides a short overview of the PDPs. Details on each PDP can be found in Appendix D. Further details on the operational interview process are provided in Appendix E. 
Based on the interviews, we created the ex-post list by validating, extending, and refining the candidate SFs from the ex-ante list (step 4). Finally, we linked the candidate SFs from the ex-post list as independent variables with relevant PDP success criteria as dependent variables (step 5). This resulted in the PDP Success Model.

Table 2 Overview of the PDPs Investigated Expert Interviews

\begin{tabular}{lll}
\hline Company & PDP & Description \\
\hline \multirow{2}{*}{ Polymer (P) } & $\begin{array}{l}\text { Digital flow } \\
\text { production (P1) }\end{array}$ & $\begin{array}{l}\text { Implementation of digital flow production to replace the manual } \\
\text { material logistics }\end{array}$ \\
\cline { 2 - 3 } Automotive (A) & $\begin{array}{l}\text { Industrial IoT } \\
\text { application (A1) }\end{array}$ & $\begin{array}{l}\text { Introduction of an industrial IoT application, replacing manual quality } \\
\text { control through automated quality control measures based on sensor data }\end{array}$ \\
\hline \multirow{3}{*}{ Healthcare (H) } & \begin{tabular}{l} 
IoT platform (H1) \\
\cline { 2 - 3 }
\end{tabular} & $\begin{array}{l}\text { Introduction of an IoT platform connecting smart devices, the } \\
\text { manufacturing enterprise system, and enterprise resource planning }\end{array}$ \\
\cline { 2 - 3 } & Maintenance (H2) & $\begin{array}{l}\text { Introduction of a predictive maintenance solution to redesign the } \\
\text { process of plant maintenance }\end{array}$ \\
\cline { 2 - 3 } & Big data analytics (H3) & $\begin{array}{l}\text { Introduction of big data analytics of energy generation and } \\
\text { consumption data }\end{array}$ \\
\hline Textile (T) & $\begin{array}{l}\text { Robotic process } \\
\text { automation (T1) }\end{array}$ & \begin{tabular}{l} 
Introduction of robotic process automation in the sales department \\
\hline
\end{tabular}
\end{tabular}

\section{Results}

In this section, we first present the ex-ante list of candidate PDP SFs (Section 4.1) After that, we present the expost list of candidate PDP SFs (Section 4.2) and the final PDP Success Model (Section 4.3).

\subsection{Ex-Ante List of Candidate Success Factors}

To group the candidate PDP SFs extracted from the literature, we first considered seminal IS success models from the literature. As none of these models specifically covers the PDP context, we reverted to frameworks from related fields such as work systems theory, BPM, and PM, which fit the interdisciplinary and socio-technical nature of our research (i.e., Aladwani, 2002; Alter, 2013; Davis, 1989; Petter et al., 2013; Rosemann and Vom Brocke, 2015). For example, Alter (2013) defines work systems as including customers, environment, information, infrastructure, participants, processes and activities, products and services, strategies, and technologies. Moreover, Petter et al. (2013) proposed a model of independent factors of IS success relying on task, project, organizational, user, and social characteristics. Rosemann and Vom Brocke (2015) introduced the six core elements of BPM (i.e., culture, governance, information technology, methods, people, and strategic alignment), which are also referred to as SFs of BPM on the enterprise level. Finally, Aladwani (2002) presents a model for IS project success, which 
highlights organizational, people, process, project, task, and technology characteristics, while Davis (1989) introduce factors affecting user acceptance of technology through perceived usefulness and ease of use.

As the categories used in these frameworks are highly overlapping, we decided to group the candidate SFs within the author team according to categories backed by multiple frameworks. To assign the identified candidate SFs to categories, we proceeded in the same way as when grouping the success codes to candidate SFs, following the proposed approach of Krippendorff (2013). Having compared multiple alternatives, we came up with a configuration of seven categories. These categories are: culture (Rosemann and Vom Brocke, 2015; Petter et al., 2013), process (Alter, 2013; Aladwani, 2002), project (Aladwani, 2002; Petter et al., 2013), people (Rosemann and Vom Brocke, 2015; Petter et al., 2013; Aladwani, 2002; Alter, 2013), strategy (Rosemann and Vom Brocke, 2015; Alter, 2013), structure (Aladwani, 2002; Petter et al., 2013; Alter, 2013), and technology (Aladwani, 2002; Alter, 2013; Davis, 1989; Rosemann and Vom Brocke, 2015). The mapping of candidate SFs to these categories resulted in the ex-ante list. Table 3 not only lists the candidate SFs, but also their definitions, justificatory references, and a rationale why the respective SFs may drive PDP success.

Overall, the strategy category ( 2 SFs; $7 \%$ ) includes factors addressing the clarity of goals and the integration of departmental digitalization strategies. Structure (2 SFs, 7\%) relies on infrastructural and organizational agility, whereas culture (5 SFs, 16\%) comprises factors referring to the working environment as well as the attitudes of different roles and individuals. The people category (7 SFs, 23\%) covers factors influencing human knowledge and skills in different areas relevant for PDPs. The process category (2 SFs, 7\%) includes selected activities from the BPM lifecycle, which were found to positively affect PDP success, while the project category (10 SFs, 33\%) emphasizes the influence of communication and selected PM activities. Finally, the technology category (2 SFs, $7 \%$ ) accounts for SF candidates that depend on the DTs employed. Overall, the project category contains the most candidate SFs followed by people and culture. The strategy, structure, process, and technology categories only contain two SFs each. Appendix F provides an overview of all success codes and justificatory references.

Table 3 The Ex-Ante List of Candidate PDP SFs Structured into Seven Categories

\begin{tabular}{|c|c|c|c|c|}
\hline Category & Name & Definition & $\begin{array}{l}\text { Justificatory } \\
\text { References }\end{array}$ & Success Rationale \\
\hline \multirow[t]{2}{*}{ Strategy } & $\begin{array}{l}\text { Goal } \\
\text { Clarity }\end{array}$ & $\begin{array}{l}\text { Transparency and consistency of } \\
\text { organizational goals }\end{array}$ & $\begin{array}{l}\text { Pankratz and } \\
\text { Loebbecke (2011) }\end{array}$ & $\begin{array}{l}\text { Enables the effective and effi- } \\
\text { cient execution of the PDP }\end{array}$ \\
\hline & $\begin{array}{l}\text { Strategy } \\
\text { Integration }\end{array}$ & $\begin{array}{l}\text { Alignment of the organization's } \\
\text { business, IT, and digital strategy }\end{array}$ & $\begin{array}{l}\text { Rodríguez et al. } \\
\text { (2020b) }\end{array}$ & $\begin{array}{l}\text { Reduces inefficiencies within } \\
\text { the PDP through implementing }\end{array}$ \\
\hline
\end{tabular}


different strategies in an organization

\begin{tabular}{|c|c|c|c|c|}
\hline \multirow[t]{2}{*}{ Structure } & $\begin{array}{l}\text { Infrastructural } \\
\text { Agility }\end{array}$ & $\begin{array}{l}\text { Extendibility, compatibility, and } \\
\text { robustness of the organization's } \\
\text { infrastructure }\end{array}$ & $\begin{array}{l}\text { Holotuik and } \\
\text { Beimborn (2017) }\end{array}$ & $\begin{array}{l}\text { Ensures adaptability to unex- } \\
\text { pected infrastructural changes } \\
\text { within the PDP }\end{array}$ \\
\hline & $\begin{array}{l}\text { Organizational } \\
\text { Agility }\end{array}$ & $\begin{array}{l}\text { The organization's overall ability and } \\
\text { willingness to cope with new } \\
\text { conditions }\end{array}$ & $\begin{array}{l}\text { Jugdev and Müller } \\
(2005)\end{array}$ & $\begin{array}{l}\text { Ensures adaptability to } \\
\text { unexpected organizational } \\
\text { changes within the PDP }\end{array}$ \\
\hline \multirow[t]{5}{*}{ Culture } & $\begin{array}{l}\text { Employee } \\
\text { Agility }\end{array}$ & $\begin{array}{l}\text { Employees' ability and willingness to } \\
\text { cope with new conditions }\end{array}$ & $\begin{array}{l}\text { Abdolvand et al. } \\
(2008)\end{array}$ & $\begin{array}{l}\text { Enables adapting to new } \\
\text { situations that emerge during } \\
\text { the PDP }\end{array}$ \\
\hline & $\begin{array}{l}\text { Innovation } \\
\text { Attitude }\end{array}$ & $\begin{array}{l}\text { The organization's open-mindedness } \\
\text { toward new developments }\end{array}$ & $\begin{array}{l}\text { Malinova et al. } \\
\text { (2014) }\end{array}$ & $\begin{array}{l}\text { Enables beneficiating from new } \\
\text { developments and opportunities } \\
\text { that can be used to promote the } \\
\text { PDP }\end{array}$ \\
\hline & $\begin{array}{l}\text { Management } \\
\text { Agility }\end{array}$ & $\begin{array}{l}\text { Management's ability and willingness } \\
\text { to cope with new conditions }\end{array}$ & $\begin{array}{l}\text { Jugdev and Müller } \\
\text { (2005) }\end{array}$ & $\begin{array}{l}\text { Enables adapting strategies and } \\
\text { the manner of leading when } \\
\text { necessary for the PDP success }\end{array}$ \\
\hline & $\begin{array}{l}\text { Resource } \\
\text { Agility }\end{array}$ & $\begin{array}{l}\text { The organization's ability and } \\
\text { willingness to dynamically } \\
\text { re-allocate resources }\end{array}$ & $\begin{array}{l}\text { Gomes and } \\
\text { Romão (2016) }\end{array}$ & $\begin{array}{l}\text { Enables in- and decreasing, or } \\
\text { shifting resources affecting the } \\
\text { business process in focus for } \\
\text { new situations }\end{array}$ \\
\hline & $\begin{array}{l}\text { Risk } \\
\text { Attitude }\end{array}$ & $\begin{array}{l}\text { The organization's willingness to take } \\
\text { risks and to experiment }\end{array}$ & $\begin{array}{l}\text { Costantino et al. } \\
\text { (2015) }\end{array}$ & $\begin{array}{l}\text { Allows for improving effective- } \\
\text { ness and efficiency of the busi- } \\
\text { ness process in focus by grasp- } \\
\text { ing opportunities }\end{array}$ \\
\hline \multirow[t]{7}{*}{ People } & $\begin{array}{l}\text { Customer } \\
\text { Knowledge }\end{array}$ & $\begin{array}{l}\text { Knowledge about the customers of the } \\
\text { business process affected by the PDP }\end{array}$ & $\begin{array}{l}\text { Holotuik and } \\
\text { Beimborn (2017) }\end{array}$ & $\begin{array}{l}\text { Enables customer-centric imple- } \\
\text { mentation of the business pro- } \\
\text { cess in focus and increases cus- } \\
\text { tomer satisfaction }\end{array}$ \\
\hline & $\begin{array}{l}\text { Employee Domain } \\
\text { Knowledge }\end{array}$ & $\begin{array}{l}\text { Employees' knowledge about and } \\
\text { experience with the domain affected } \\
\text { by the PDP }\end{array}$ & $\begin{array}{l}\text { Pankratz and } \\
\text { Loebbecke (2011) }\end{array}$ & $\begin{array}{l}\text { Ensures knowledge required to } \\
\text { correctly analyze the context of } \\
\text { the PDP }\end{array}$ \\
\hline & $\begin{array}{l}\text { Employee } \\
\text { Technology } \\
\text { Knowledge } \\
\end{array}$ & $\begin{array}{l}\text { Employees' knowledge about and } \\
\text { experience with the technologies used } \\
\text { in the PDP }\end{array}$ & $\begin{array}{l}\text { Dezdar and Ainin } \\
(2011)\end{array}$ & $\begin{array}{l}\text { Ensures knowledge required to } \\
\text { correctly use the DT affecting } \\
\text { the business process in focus }\end{array}$ \\
\hline & $\begin{array}{l}\text { Management } \\
\text { Domain } \\
\text { Knowledge } \\
\end{array}$ & $\begin{array}{l}\text { Management's knowledge about and } \\
\text { experience with the domain affected } \\
\text { by the PDP }\end{array}$ & $\begin{array}{l}\text { Irvine and Hall } \\
(2015)\end{array}$ & $\begin{array}{l}\text { Ensures management } \\
\text { knowledge required to correctly } \\
\text { analyze the context of the PDP }\end{array}$ \\
\hline & $\begin{array}{l}\text { Management } \\
\text { Technology } \\
\text { Knowledge }\end{array}$ & $\begin{array}{l}\text { Management's knowledge about and } \\
\text { experience with the technologies used } \\
\text { in the PDP }\end{array}$ & $\begin{array}{l}\text { Hughes et al. } \\
\text { (2017) }\end{array}$ & $\begin{array}{l}\text { Ensures management } \\
\text { knowledge required about the } \\
\text { DT affecting the business pro- } \\
\text { cess in focus }\end{array}$ \\
\hline & $\begin{array}{l}\text { Process } \\
\text { Improvement } \\
\text { Skills } \\
\end{array}$ & $\begin{array}{l}\text { Ability to analyze and improve } \\
\text { business processes }\end{array}$ & $\begin{array}{l}\text { Abollado et al. } \\
(2017)\end{array}$ & $\begin{array}{l}\text { Ensures methodical knowledge } \\
\text { required to improve the business } \\
\text { process in focus }\end{array}$ \\
\hline & $\begin{array}{l}\text { Process } \\
\text { Knowledge }\end{array}$ & $\begin{array}{l}\text { Knowledge about the business } \\
\text { process affected by the PDP }\end{array}$ & $\begin{array}{l}\text { Malinova et al. } \\
\text { (2014) }\end{array}$ & $\begin{array}{l}\text { Enables improving effectiveness } \\
\text { or efficiency of the affected } \\
\text { business process }\end{array}$ \\
\hline \multirow[t]{2}{*}{ Process } & Process Design & $\begin{array}{l}\text { Availability of a model for the } \\
\text { business process affected by the PDP }\end{array}$ & $\begin{array}{l}\text { Holotuik and } \\
\text { Beimborn (2017) }\end{array}$ & $\begin{array}{l}\text { Discloses potentials for digitali- } \\
\text { zation by visualizing the } \\
\text { affected business process }\end{array}$ \\
\hline & $\begin{array}{l}\text { Process } \\
\text { Monitoring }\end{array}$ & $\begin{array}{l}\text { Continuous monitoring of the busi- } \\
\text { ness process affected by the PDP }\end{array}$ & $\begin{array}{l}\text { Pankratz and } \\
\text { Loebbecke (2011) }\end{array}$ & $\begin{array}{l}\text { Discloses potentials for im- } \\
\text { provement of the affected } \\
\text { business process }\end{array}$ \\
\hline Project & $\begin{array}{l}\text { Customer } \\
\text { Integration }\end{array}$ & Integration of customers into the PDP & $\begin{array}{l}\text { Fowler and Horan } \\
(2007)\end{array}$ & $\begin{array}{l}\text { Enables early adaptation of the } \\
\text { business process in focus to a } \\
\text { customer's needs }\end{array}$ \\
\hline
\end{tabular}




\begin{tabular}{|c|c|c|c|}
\hline $\begin{array}{l}\text { Employee } \\
\text { Support }\end{array}$ & $\begin{array}{l}\text { Employees' commitment toward the } \\
\text { PDP }\end{array}$ & $\begin{array}{l}\text { Abdolvand et al. } \\
(2008)\end{array}$ & $\begin{array}{l}\text { Increases performance and re- } \\
\text { duces friction with employees } \\
\text { affected by the PDP }\end{array}$ \\
\hline $\begin{array}{l}\text { External } \\
\text { Communication }\end{array}$ & $\begin{array}{l}\text { Information and knowledge sharing } \\
\text { beyond the PDP }\end{array}$ & Ram et al. (2013) & $\begin{array}{l}\text { Improves decision-making af- } \\
\text { fecting the business process in } \\
\text { focus contingent on external } \\
\text { information }\end{array}$ \\
\hline $\begin{array}{l}\text { Internal } \\
\text { Communication }\end{array}$ & $\begin{array}{l}\text { Information and knowledge sharing } \\
\text { within the PDP }\end{array}$ & $\begin{array}{l}\text { Kirsch and } \\
\text { Slaughter (2013) }\end{array}$ & $\begin{array}{l}\text { Improves decision-making af- } \\
\text { fecting the business process in } \\
\text { focus contingent on internal in- } \\
\text { formation }\end{array}$ \\
\hline Partner Integration & Integration of partners into the PDP & Ram et al. (2013) & $\begin{array}{l}\text { Allows for beneficiating from a } \\
\text { partner's knowledge and skills } \\
\text { about the DT or business pro- } \\
\text { cess in focus }\end{array}$ \\
\hline $\begin{array}{l}\text { Project } \\
\text { Monitoring }\end{array}$ & $\begin{array}{l}\text { Continuous monitoring of the PDP's } \\
\text { performance and progress }\end{array}$ & $\begin{array}{l}\text { Frey and } \\
\text { Buxmann (2012) }\end{array}$ & $\begin{array}{l}\text { Enables adjustments during the } \\
\text { PDP to increase performance } \\
\text { and foster the PDP's progress }\end{array}$ \\
\hline $\begin{array}{l}\text { Project } \\
\text { Preparation }\end{array}$ & Detailed planning of the PDP & $\begin{array}{l}\text { Kirsch and } \\
\text { Slaughter (2013) }\end{array}$ & $\begin{array}{l}\text { Improves the later execution of } \\
\text { the PDP and anticipation of } \\
\text { challenges and opportunities of } \\
\text { the PDP }\end{array}$ \\
\hline $\begin{array}{l}\text { Team } \\
\text { Portfolio }\end{array}$ & $\begin{array}{l}\text { The PDP team's compilation of indi- } \\
\text { vidual skills and personal relations }\end{array}$ & Ram et al. (2013) & $\begin{array}{l}\text { Ensures the fit of the knowledge } \\
\text { and skills of the PDP's team } \\
\text { members }\end{array}$ \\
\hline $\begin{array}{l}\text { Team } \\
\text { Support }\end{array}$ & $\begin{array}{l}\text { The PDP team's motivation in and } \\
\text { championing of the PDP }\end{array}$ & $\begin{array}{l}\text { Gomes and } \\
\text { Romão (2016) }\end{array}$ & $\begin{array}{l}\text { Increases performance and } \\
\text { reduces friction within the PDP }\end{array}$ \\
\hline $\begin{array}{l}\text { Top Management } \\
\text { Support }\end{array}$ & $\begin{array}{l}\text { Management's commitment to and } \\
\text { championing of the PDP }\end{array}$ & $\begin{array}{l}\text { Costantino et al. } \\
(2015)\end{array}$ & $\begin{array}{l}\text { Fosters employee's motivation } \\
\text { and enables a holistic assess- } \\
\text { ment of the PDP }\end{array}$ \\
\hline $\begin{array}{l}\text { Technology } \\
\text { Complexity }\end{array}$ & $\begin{array}{l}\text { Effort needed to implement, run, and } \\
\text { execute the technologies used in the } \\
\text { PDP }\end{array}$ & $\begin{array}{l}\text { Hughes et al. } \\
(2017)\end{array}$ & $\begin{array}{l}\text { Reduces skillset and preparation } \\
\text { needed to implement the DT in } \\
\text { focus }\end{array}$ \\
\hline $\begin{array}{l}\text { Technology } \\
\text { Maturity }\end{array}$ & $\begin{array}{l}\text { Readiness-for-use of the technologies } \\
\text { used in the PDP }\end{array}$ & $\begin{array}{l}\text { Irvine and Hall } \\
(2015)\end{array}$ & $\begin{array}{l}\text { Reduces efforts needed to } \\
\text { implement the DT in focus }\end{array}$ \\
\hline
\end{tabular}

\subsection{Ex-Post List of Candidate Success Factors}

While conducting the interviews, we identified seven new candidate SFs and refined two further candidates into three new ones. Moreover, we found preliminarily support for the influence of 19 candidate SFs from the ex-ante list, as these candidate SFs have been actively mentioned by the interviewees. Interestingly, there were nine candidate SFs identified from the literature for which we did not find any empirical support. Based on the refinement and validation of the ex-ante list, we included the supported, new, and refined SFs into the ex-post list. We also included the SF candidates for which we could not find empirical support owing to the exploratory nature of our research, i.e., we cannot exclude a positive effect on PDP success with certainty only based on the interviews. Table 4 provides an overview. In case of new and refined SFs, it also includes the (refined) definition and justificatory references, which we identified while critically reflecting on these findings. Details on new SFs as well as the refinement of existing SFs can be found in Appendix G (new SFs) and Appendix H (refined SFs). 
Table 4 New, Refined, and Candidate PDP SFs without Supporting Evidence

\begin{tabular}{|c|c|c|c|c|c|}
\hline Category & Name & Attribute & (Refined) Definition & $\begin{array}{l}\text { Justificatory } \\
\text { References } \\
\end{array}$ & Success Rationale \\
\hline Strategy & $\begin{array}{l}\text { Digital } \\
\text { Ambition }\end{array}$ & New & $\begin{array}{l}\text { Continuous focus on the digital- } \\
\text { ization of the organization and } \\
\text { its processes }\end{array}$ & Gartner (2017) & $\begin{array}{l}\text { Enhances team mem- } \\
\text { bers motivation and } \\
\text { commitment and in- } \\
\text { creases the PDP's effec- } \\
\text { tiveness and efficiency }\end{array}$ \\
\hline Structure & $\begin{array}{l}\text { Organizational } \\
\text { Agility }\end{array}$ & $\begin{array}{l}\text { No supporting } \\
\text { evidence found }\end{array}$ & - & - & \\
\hline \multirow{2}{*}{ Culture } & $\begin{array}{l}\text { Digitalization } \\
\text { Attitude }\end{array}$ & New & $\begin{array}{l}\text { PDP members' willingness-to- } \\
\text { change and open-mindedness } \\
\text { toward DTs }\end{array}$ & Koleva (2019) & $\begin{array}{l}\text { Facilitates the integra- } \\
\text { tion of the DT into the } \\
\text { business process in fo- } \\
\text { cus }\end{array}$ \\
\hline & Partner Agility & New & $\begin{array}{l}\text { Partners' ability and willingness } \\
\text { to cope with new conditions }\end{array}$ & Ren et al. (2005) & $\begin{array}{l}\text { Enables adapting to new } \\
\text { situations within PDP's } \\
\text { processes that involve } \\
\text { partners }\end{array}$ \\
\hline \multirow{6}{*}{ People } & Data Analysis & New & $\begin{array}{l}\text { Usage of analytical and deci- } \\
\text { sion-making capabilities for di- } \\
\text { agnostic, descriptive, prescrip- } \\
\text { tive, and predictive purposes }\end{array}$ & $\begin{array}{l}\text { Porter and } \\
\text { Heppelmann } \\
(2015)\end{array}$ & $\begin{array}{l}\text { Facilitates beneficial us- } \\
\text { age of data analysis } \\
\text { technologies in the busi- } \\
\text { ness process in focus }\end{array}$ \\
\hline & $\begin{array}{l}\text { Partner Domain } \\
\text { Knowledge }\end{array}$ & New & $\begin{array}{l}\text { Partners' knowledge about and } \\
\text { experience with the domain af- } \\
\text { fected by the PDP }\end{array}$ & $\begin{array}{l}\text { Yayavaram et } \\
\text { al. (2018) }\end{array}$ & $\begin{array}{l}\text { Ensures partner } \\
\text { knowledge required to } \\
\text { correctly analyze the } \\
\text { context of the PDP }\end{array}$ \\
\hline & $\begin{array}{l}\text { Partner Technol- } \\
\text { ogy Knowledge }\end{array}$ & New & $\begin{array}{l}\text { Partners' knowledge about and } \\
\text { experience with the technolo- } \\
\text { gies used in the PDP }\end{array}$ & $\begin{array}{l}\text { Flor et al. } \\
(2018)\end{array}$ & $\begin{array}{l}\text { Ensures partner } \\
\text { knowledge required } \\
\text { about the DT affecting } \\
\text { the business process in } \\
\text { focus }\end{array}$ \\
\hline & $\begin{array}{l}\text { Customer } \\
\text { Knowledge } \\
\end{array}$ & $\begin{array}{l}\text { No supporting } \\
\text { evidence found }\end{array}$ & - & - & \\
\hline & $\begin{array}{l}\text { Management Do- } \\
\text { main Knowledge }\end{array}$ & $\begin{array}{l}\text { No supporting } \\
\text { evidence found }\end{array}$ & - & - & \\
\hline & $\begin{array}{l}\text { Management } \\
\text { Technology } \\
\text { Knowledge } \\
\end{array}$ & $\begin{array}{l}\text { No supporting } \\
\text { evidence found }\end{array}$ & - & - & \\
\hline \multirow[t]{3}{*}{ Process } & $\begin{array}{l}\text { Process Goal } \\
\text { Clarity }\end{array}$ & Refined & $\begin{array}{l}\text { Transparency and consistency } \\
\text { of the goals of the business pro- } \\
\text { cess affected by the PDP }\end{array}$ & $\begin{array}{l}\text { Peralta et al. } \\
(2015)\end{array}$ & $\begin{array}{l}\text { Facilitates modifying } \\
\text { the business process in } \\
\text { focus in such a way that } \\
\text { it brings the desired out- } \\
\text { come to the company }\end{array}$ \\
\hline & Process Design & $\begin{array}{l}\text { No supporting } \\
\text { evidence found }\end{array}$ & - & - & \\
\hline & $\begin{array}{l}\text { Process Monitor- } \\
\text { ing }\end{array}$ & $\begin{array}{l}\text { No supporting } \\
\text { evidence found }\end{array}$ & - & - & \\
\hline \multirow[t]{2}{*}{ Project } & $\begin{array}{l}\text { Project Goal Clar- } \\
\text { ity }\end{array}$ & Refined & $\begin{array}{l}\text { Transparency and consistency } \\
\text { of the PDP goals }\end{array}$ & $\begin{array}{l}\text { Raziq et al. } \\
(2018)\end{array}$ & $\begin{array}{l}\text { Facilitates finding a lean } \\
\text { and efficient path to the } \\
\text { PDP's goal }\end{array}$ \\
\hline & $\begin{array}{l}\text { Project Monitor- } \\
\text { ing }\end{array}$ & $\begin{array}{l}\text { No supporting } \\
\text { evidence found }\end{array}$ & - & - & \\
\hline $\begin{array}{l}\text { Technol- } \\
\text { ogy }\end{array}$ & $\begin{array}{l}\text { Technology } \\
\text { Comprehensibility }\end{array}$ & New & $\begin{array}{l}\text { The level of abstractness of the } \\
\text { DT used in the PDP }\end{array}$ & $\begin{array}{l}\text { Flor et al. } \\
(2018)\end{array}$ & $\begin{array}{l}\text { Enables understanding } \\
\text { the functionality of the } \\
\text { DT in focus and how to } \\
\text { use it in the affected } \\
\text { business process }\end{array}$ \\
\hline
\end{tabular}




\begin{tabular}{llll}
\hline $\begin{array}{l}\text { Infrastructural } \\
\text { Readiness }\end{array}$ & Refined & $\begin{array}{l}\text { Extendibility, compatibility, and } \\
\text { robustness of the organization's } \\
\text { technological infrastructure }\end{array}$ & $\begin{array}{l}\text { Facilitates successful } \\
\text { (2018) }\end{array}$ \\
\hline $\begin{array}{l}\text { Technology } \\
\text { Complexity }\end{array}$ & $\begin{array}{l}\text { No Supporting } \\
\text { Evidence found }\end{array}$ & $\begin{array}{l}\text { implentation of the } \\
\text { DT focus within the } \\
\text { existing infrastructure }\end{array}$ \\
\hline $\begin{array}{l}\text { Technology } \\
\text { Maturity }\end{array}$ & $\begin{array}{l}\text { No Supporting } \\
\text { Evidence found }\end{array}$ & - & - \\
\hline
\end{tabular}

\subsection{PDP Success Model}

In line with the results from the expert interviews, we built the ex-post list of candidate PDP SFs. The final step was to compile the PDP Success Model by linking the candidate PDP SFs from the ex-post list as independent variables with PDP success criteria as dependent variables. For our purposes, we used project efficiency and project effectiveness as success criteria, as they are commonly used in the general PM literature (Drucker, 2007; Beer et al., 2013) as well as for measuring the success of process change initiatives (Bandara et al., 2005; Schmiedel et al., 2020). Moreover, effectiveness and efficiency are specifically well-known in the exploitative BPM literature (Grisold et al., 2019), which is why they fit our PDP definition very well. The final PDP Success Model, which includes 38 SFs structured according to seven categories, is presented in Figure 2. 


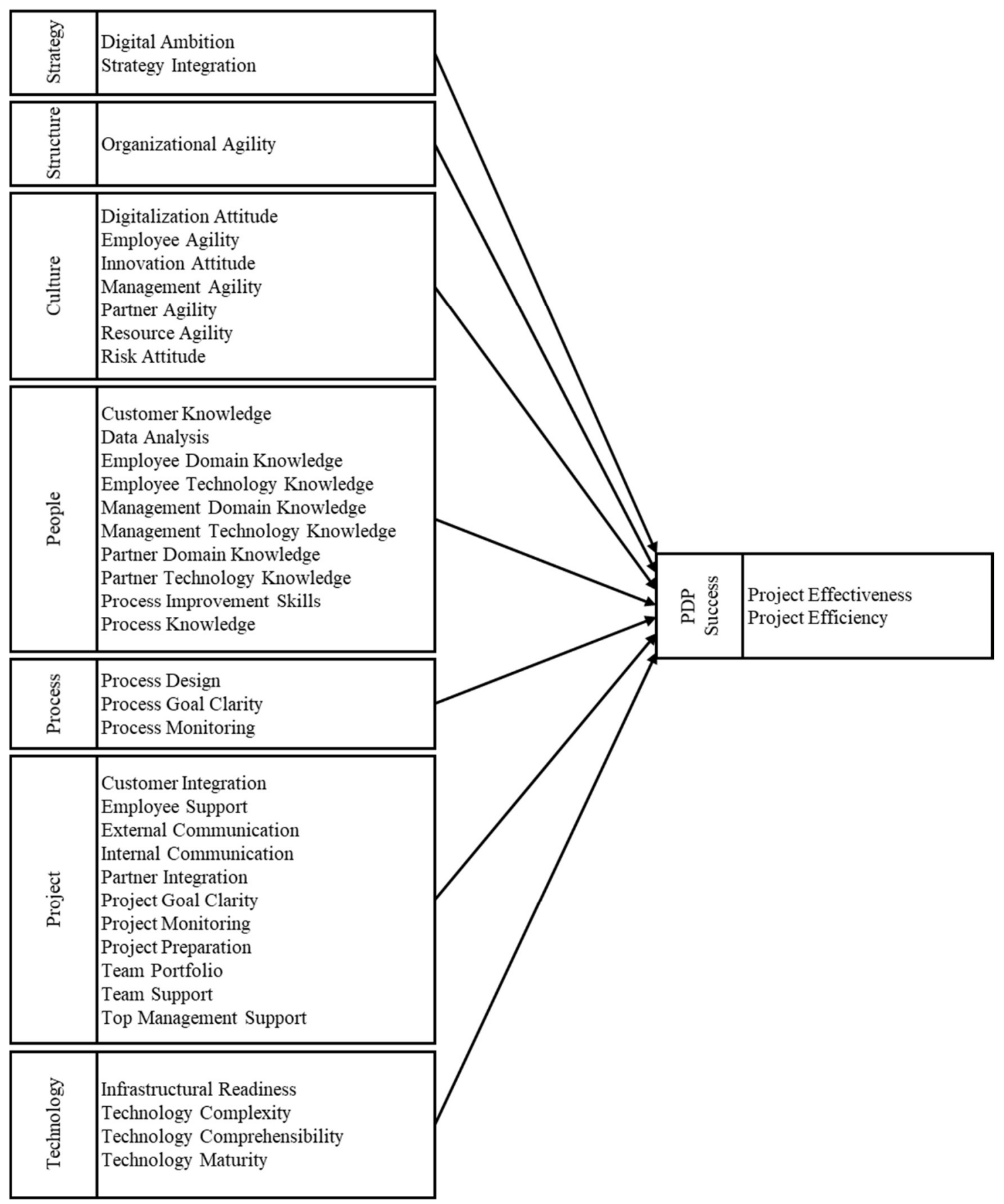

Fig. 2 The PDP Success Model 


\section{Discussion and Conclusion}

\subsection{Contribution}

Our study was motivated by the lack of knowledge on how organizations can leverage DTs to improve and innovate business processes. While there are methods and tools for identifying process digitalization ideas and projects, guidance on the successful implementation of PDPs is missing. Hence, we set out to explore factors that drive PDP success. We conducted a structured literature review to integrate isolated pockets of understanding as well as semistructured interviews to validate, refine, and extend these results based on first-hand insights. Our key contribution is the PDP Success Model, which links 38 candidate SFs distributed across seven literature-backed categories with PDP success criteria. We also provided preliminary success rationales that support the sense-making process and can be used as foundation for future research.

\subsection{Theoretical Implications}

From a theoretical perspective, our study is the first to link the BPM, PM, and digitalization fields, whereas the intersection of pairs of these fields has already been studied, e.g., the intersection of BPM and PM (Lehnert et al., 2016; Limam Mansar et al., 2009; Darmani and Hanafizadeh, 2013) or the intersection of BPM and digitalization (Kerpedzhiev et al., 2020; Beverungen et al., 2020; Baiyere et al., 2020). Moreover, our study is the first to investigate SFs for PDPs, which is essential given the impact of digitalization on BPM (Kerpedzhiev et al., 2020) and the importance of business process digitalization for corporate success (Denner et al., 2018; Rosemann, 2020).

The PDP Success Model implies that the SFs currently discussed in the BPM, PM, and digitalization literature need refinement and extension to cover the particularities of PDPs. This becomes manifest in the high number of newly identified and refined candidate SFs. Ten out of the 38 candidate SFs from the ex-post list across all categories emerged during the interviews (e.g., digital ambition, partner agility, and partner analysis), meaning that they were not discussed in relation to process digitalization so far. Moreover, nine candidate SFs from the ex-ante list remained without empirical support (e.g., organizational agility, management technology knowledge, and process design). This circumstance indicates that the originally found effect of these candidate SFs may not apply to PDPs - at least not to those from our sample. At the same time, 28 candidate SFs from the PDP Success Model are already backed by the literature. Hence, digitalization questions existing knowledge but it does not render it 
obsolete. Moreover, PDPs require a unique set of SFs, which combine established and hitherto underrepresented knowledge from the BPM, PM, and digitalization fields.

Our results foster the understanding of PDP success and extend papers that already dealt with business process digitalization. First, the PDP Success Model complements methods and tools for identifying process digitalization ideas and PDPs (Denner et al., 2018; Rosemann, 2020). By nature, such approaches neglect the implementation phase of PDPs. Our results also complement studies focusing on BPM SFs (Trkman, 2010; Rosemann and Vom Brocke, 2015; Castro et al., 2020), which take an enterprise-level perspective abstracting from individual projects. Most specifically, our results build on, update, and extend those studies that investigated SFs on the level of individual projects (Al-Mashari and Zairi, 1999; McLean and Antony, 2014). Owing to their publication date, these works do not account for the challenges and opportunities brought about by digitalization (Legner et al., 2017). Our findings comply with Kerpedzhiev et al. (2020), who stated that BPM in the digital age calls for different capabilities, while we found that capabilities on the project level have changed as well. In addition, some existing works focus on SFs and pitfalls of specific activities within process projects, such as process modeling (Bandara et al., 2005; Rosemann, 2006). Compared to these papers, the PDP Success Model is not limited to specific activities and takes a broad perspective as expressed in terms of the covered categories.

\subsection{Managerial Implications}

From a managerial perspective, the PDP Success Model has the potential to guide PDP managers and their teams when planning and conducting PDPs. This leads to the following operational implications:

Managers should account for relevant SFs when conducting PDP and assess their PDPs accordingly. PDP teams can use the PDP Success Model as a foundation for fit/gap analyses and self-assessments. Using a SWOT analysis, for example, managers can monitor their PDP to exploit strengths and opportunities as well as eliminate threats and weaknesses. These insights can also be used for continuous benefits management. PDP managers can also use the PDP Success Model to assess the extent to which certain candidate SFs can be influenced to sensibly allocate scarce team resources and management attention. If such activities are performed repeatedly, PDP teams can develop an awareness of relevant PDP SFs and become consciously competent in successfully completing PDPs.

Managers should be sensitive to new findings and overcome old patterns of thought. Using open innovation methods, managers can integrate external knowledge and identify new ideas as well as SFs for the successful 
implementation of PDPs. The fact that one quarter of the candidate SFs from the PDP Success Model emerged during the interviews and that we could not find empirical support for another nine SFs during the interviews should sensitize PDP teams that they must not blindly trust their experience and what they have learned in the past. Rather, PDP teams should pay particular attention to the newly identified candidate SFs and those without empirical support. It is regarding these SFs where existing PM routines must be adjusted.

Managers should understand the interplay of BPM, PM, and digitalization to prepare for new requirements. As digitalization brings about new challenges and opportunities, hitherto established ways of realizing process change need refinement. Our study showed that current SFs from the literature may not be applicable to PDPs anymore. During the interviews, we identified that three out of seven new SF candidates relate to digitalization (i.e., digital ambition, digitalization attitude, and data analysis). Digitalization offers new ways to design business processes and evaluate their performance. Hence, managers should consider these aspects related to digitalization when planning for the successful implementation of PDPs.

\subsection{Limitations and Future Research}

Our findings must be interpreted considering certain limitations, which also stimulate future research. First, to fully understand PDP success, it is important to also account for interactions among SFs. This is worthwhile, as research in related fields discovered relations among SFs (Petter et al., 2013; Guimaraes, 1997). For the purposes of our exploratory study, we treated the candidate SFs as independent and equally important. Furthermore, we kept candidate SFs from the literature in the presented PDP Success Model even if we could not find empirical evidence for them during the interviews. The reason is that we cannot exclude a positive effect on PDP success with certainty owing to the exploratory nature of our research. Hence, the number of candidate SFs included in the PDP Success Model is rather high. In line with our exploratory approach, we used the literature from BPM, PM, and digitalization as central source of evidence. Although we conducted the literature review with due care, we cannot guarantee that we did not miss out single studies. Moreover, to group the identified SF candidates, we drew from wellestablished frameworks. We examined the categorization qualitatively and quantitatively and tested different alternatives. However, the final categorization has been made by the author team, which is why future research may investigate alternative groupings of the SF candidates. 
Second, our definition of PDPs covers exploitative process change and explorative BPM that seizes opportunities to reengineer existing processes. It excludes explorative BPM geared toward the opportunity-led creation of novel processes. Accordingly, we chose the PDPs covered in the interviews to fit this definition. Hence, the transferability of our results may be limited in this regard. As explorative process change features a close relationship with innovation management and does not build on existing processes, related PDPs may require different SFs and maybe even different success criteria. Finally, the PDPs covered during the interviews were limited to the businessto-business domain. While we searched the related BPM, PM, and digitalization literature without restrictions, we limited ourselves to the manufacturing domain for the expert interviews. Although this was a deliberate design decision of the author team, we cannot exclude whether interviews in other domains would have led us to identify different new candidate SFs.

Future research should address the limitations and take our results one step further. As for the limitations, future research should challenge the PDP Success Model through literature reviews and interviews in other domains. Related findings may provide useful hints for contextualizing the PDP Success Model. Moreover, future research should investigate SFs and success criteria for explorative PDPs with a focus on the creation of new business processes. As our research had an exploratory focus, the explanatory power of the PDP Success Model yet needs to be investigated through confirmatory research (e.g., surveys). To that end, future research should account for potential interactions among SFs within the same category and across categories. To account for the diverse domains in which PDPs are performed, context should be included as a moderating variable. To that end, future research may leverage the BPM context framework (Vom Brocke et al., 2016), which has been successfully used for BPM method assessment and selection (Vom Brocke et al., 2020), as well as the characteristics of digital technologies to account for different types of DTs (Berger et al., 2018; Benbya et al., 2020). Finally, future research may take a closer look at those candidate SFs for which we could not find supporting empirical evidence during the interviews, as they need special scrutiny whether they really apply to PDPs. Even more, the same holds true for those SF candidates that emerged during the interviews. Their definitions should be substantiated, and it should be investigated whether they also shape up useful in domains beyond PDPs. 


\section{References}

Abdelkafi, N. and Pero, M. (2018), "Supply chain innovation-driven business models", Business Process Management Journal, Vol. 24 No. 2, pp. 589-608.

Abdolvand, N., Albadvi, A. and Ferdowsi, Z. (2008), "Assessing readiness for business process reengineering”, Business Process Management Journal, Vol. 14 No. 4, pp. 497-511.

Abollado, J.R., Shehab, E. and Bamforth, P. (2017), "Challenges and Benefits of Digital Workflow Implementation in Aerospace Manufacturing Engineering", Procedia CIRP, Vol. 60, pp. 80-85.

Adumene, S., Khan, F., Adedigba, S. and Zendehboudi, S. (2021), "Offshore system safety and reliability considering microbial influenced multiple failure modes and their interdependencies", Reliability Engineering \& System Safety, Vol. 215, p. 107862.

Ahmed, S. (2021), “Artificial intelligence and machine learning for process safety: Points to ponder”, Process Safety Progress, Vol. 40 No. 4, pp. 189-190.

Aladwani, A.M. (2002), “An Integrated Performance Model Information Systems Projects”, Journal of Management Information Systems, Vol. 19 No. 1, pp. 185-210.

Allen, M., Alleyne, D., Farmer, C., McRae, A. and Turner, C. (2014), “A framework for project success”, Journal of Information Technology and Economic Development, Vol. 5 No. 2, p. 1.

Al-Mashari, M. and Zairi, M. (1999), "BPR implementation process. An analysis of key success and failure factors", Business Process Management Journal, Vol. 5 No. 1, pp. 87-112.

Alter, S. (2013), "Work system theory. Overview of core concepts, extensions, and challenges for the future", Journal of the Association for Information Systems, Vol. 14 No. 2, p. 72.

Antony, J., Krishan, N., Cullen, D. and Kumar, M. (2012), "Lean Six Sigma for higher education institutions (HEIs)", International Journal of Productivity and Performance Management, Vol. 61 No. 8, pp. 940-948.

Archibald, R.D. (2003), Managing high-technology programs and projects, 3rd ed., John Wiley \& Sons, Inc., Hoboken, New Jersey.

Arthur, W.B. (2009), The nature of technology: What it is and how it evolves, Simon and Schuster.

Arunthavanathan, R., Khan, F., Ahmed, S. and Imtiaz, S. (2021), "A deep learning model for process fault prognosis”, Process Safety and Environmental Protection, Vol. 154, pp. 467-479.

Avital, M. (2003), "Reexamining Information Systems Success through the Information Technology Professionals Perspective 1", Sprouts: Working Papers on Information Environments, Systems and Organizations, Vol. 3.

Bai, C. and Sarkis, J. (2013), “A grey-based DEMATEL model for evaluating business process management critical success factors", International Journal of Production Economics, Vol. 146 No. 1, pp. 281-292.

Baiyere, A., Salmela, H. and Tapanainen, T. (2020), "Digital transformation and the new logics of business process management", European Journal of Information Systems, Vol. 29 No. 3, pp. 238-259.

Bandara, W., Gable, G.G. and Rosemann, M. (2005), "Factors and measures of business process modelling: model building through a multiple case study", European Journal of Information Systems, Vol. 14 No. 4, pp. $347-360$.

Baskerville, R., Myers, M.D. and Yoo, Y. (2020), "Digital First. The Ontological Reversal and New Challenges for Information Systems Research”, MIS Quarterly, Vol. 44 No. 2, pp. 509-523.

Beer, M., Fridgen, G., Mueller, H.-V. and Wolf, T. (2013), "Benefits Quantification in IT Projects", Wirtschaftsinformatik Proceedings 2013 No. 45, pp. 707-720.

Benbasat, I., Goldstein, D.K. and Mead, M. (1987), “The Case Research Strategy in Studies of Information Systems", MIS Quarterly, Vol. 11 No. 3, p. 369. 
Benbya, H., Nan, N., Tanriverdi, H. and and Yoo, Y. (2020), "Complexity and Information Systems Research in the Emerging Digital World”, MIS Quarterly, Vol. 44 No. 1, pp. 1-17.

Berger, S., Denner, M.-S. and Roeglinger, M. (2018), “The Nature of Digital Technologies-Development of a Multi-Layer Taxonomy", in Proceedings of the 26th European Conference on Information Systems, pp. 118.

Beringer, C., Jonas, D. and Kock, A. (2013), "Behavior of internal stakeholders in project portfolio management and its impact on success", International Journal of Project Management, Vol. 31 No. 6, pp. 830-846.

Besteiro, E.N., Pinto, J.d. and Novaski, O. (2015), "Success factors in project management", Business Management Dynamics, Vol. 4 No. 9, pp. 19-34.

Beverungen, D., Buijs, J.C.A.M., Becker, J., Di Ciccio, C., van der Aalst, W.M.P., Bartelheimer, C., Vom Brocke, J., Comuzzi, M., Kraume, K., Leopold, H., Matzner, M., Mendling, J., Ogonek, N., Post, T., Resinas, M., Revoredo, K., del-Río-Ortega, A., La Rosa, M., Santoro, F.M., Solti, A., Song, M., Stein, A., Stierle, M. and Wolf, V. (2020), "Seven Paradoxes of Business Process Management in a Hyper-Connected World”, Business \& Information Systems Engineering, Vol. 18 No. 2, p. 279.

Beverungen, D., Müller, O., Matzner, M., Mendling, J. and Vom Brocke, J. (2019), “Conceptualizing smart service systems", Electronic Markets, Vol. 29 No. 1, pp. 7-18.

Bhattacherjee, A. (2012), Social science research: Principles, methods, and practices, Textbooks Collection, Vol. 3, 2nd ed., University of South Florida, Tampa, FL.

Bradley, J. (2005), “Are All Critical Sucess Factors in ERP Implementation Created Equal?”, AMCIS 2005 Proceedings, p. 158.

Bullen, C.V. and Rockart, J.F. (1981), “A primer on critical success factors”.

Castro, B.K.d.A., Dresch, A. and Veit, D.R. (2020), "Key critical success factors of BPM implementation: a theoretical and practical view”, Business Process Management Journal, Vol. 26 No. 1, pp. 239-256.

Clarke, A. (1999), "A practical use of key success factors to improve the effectiveness of project management", International Journal of Project Management, Vol. 17 No. 3, pp. 139-145.

Clarke, R. (2016), "Big data, big risks”, Information Systems Journal, Vol. 26 No. 1, pp. 77-90.

Cooke-Davies, T. (2002), “The "real” success factors on projects", International Journal of Project Management, Vol. 20 No. 3, pp. 185-190.

Corbin, J. and Strauss, A. (1990), "Grounded Theory Research. Procedures, canons, and evaluative Criteria”, Qualitative Sociology, Vol. 13 No. 1, pp. 3-21.

Costantino, F., Di Gravio, G. and Nonino, F. (2015), "Project selection in project portfolio management: An artificial neural network model based on critical success factors", International Journal of Project Management, Vol. 33 No. 8, pp. 1744-1754.

Crouch, M. and McKenzie, H. (2006), “The logic of small samples in interview-based qualitative research”, Social Science Information, Vol. 45 No. 4, pp. 483-499.

Darmani, A. and Hanafizadeh, P. (2013), "Business process portfolio selection in re-engineering projects", Business Process Management Journal, Vol. 19 No. 6, pp. 892-916.

Daugherty, P. (2020), "Managing Technology for the Post-Digital Era”, MIT sloan management review, 2020, available at: https://sloanreview.mit.edu/article/managing-technology-for-the-post-digital-era/ (accessed Retrieved 16 April 2020).

Davenport, T.H. and Westerman, G. (2018), "Why so many high-profile digital transformations fail”, Harvard Business Review, 2018, available at: https://hbr.org/2018/03/why-so-many-high-profile-digital-transformations-fail (accessed Retrieved at 16 April, 2020). 
Davis and Hufnagel (2007), "Through the Eyes of Experts: A Socio-Cognitive Perspective on the Automation of Fingerprint Work", MIS Quarterly, Vol. 31 No. 4, p. 681.

Davis, F.D. (1989), "Perceived Usefulness, Perceived Ease of Use, and User Acceptance of Information Technology”, MIS Quarterly, Vol. 13 No. 3, p. 319.

de Reyck, B., Grushka-Cockayne, Y., Lockett, M., Calderini, S.R., Moura, M. and Sloper, A. (2005), "The impact of project portfolio management on information technology projects", International Journal of Project Management, Vol. 23 No. 7, pp. 524-537.

Denner, M.-S., Püschel, L. and Roeglinger, M. (2018), "How to Exploit the Digitalization Potential of Business Processes", Business \& Information Systems Engineering, Vol. 60 No. 4, pp. 331-349.

Dezdar, S. (2012), "Strategic and tactical factors for successful ERP projects: insights from an Asian country", Management Research Review, Vol. 35 No. 11, pp. 1070-1087.

Dezdar, S. and Ainin, S. (2011), "Examining ERP implementation success from a project environment perspective”, Business Process Management Journal, Vol. 17 No. 6, pp. 919-939.

Drucker, P.F. (2007), People and performance: The best of Peter Drucker on management, 2nd ed., Harvard Business School Press, Boston, Mass.

Dumas, M., La Rosa, M., Mendling, J. and Reijers, H.A. (2018), Fundamentals of Business Process Management, Springer-Verlag, Berlin, Heidelberg.

Dvir, D., Lipovetsky, S., Shenhar, A. and Tishler, A. (1998), "In search of project classification. A non-universal approach to project success factors", Research Policy, Vol. 27 No. 9, pp. 915-935.

Fitzgerald, M., Kruschwitz, N., Bonnet, D. and Welch, M. (2014), "Embracing digital technology. A new strategic imperative", MIT sloan management review, Vol. 55 No. 2, p. 1.

Flor, M.L., Cooper, S.Y. and Oltra, M.J. (2018), "External knowledge search, absorptive capacity and radical innovation in high-technology firms", European Management Journal, Vol. 36 No. 2, pp. 183-194.

Fowler, J.J. and Horan, P. (2007), “Are information systems' success and failure factors related? An exploratory study”, Journal of Organizational and End User Computing, Vol. 19 No. 2, p. 1.

Frey, T. and Buxmann, P. (2012), "IT project portfolio management - a structured literature review", in Proceedings of the 20th European Conference on Information Systems, pp. 1-25.

Galliers, R.D. and Huang, J.C. (2012), "The teaching of qualitative research methods in information systems: an explorative study utilizing learning theory”, European Journal of Information Systems, Vol. 21 No. 2, pp. $119-134$.

Gartner (2017), Digital Business Ambition: Transform or Optimize?, Gartner, Inc, Stamford, CT.

Gartner (2020), Hype Cycle for Emerging Technologies: 2020, Gartner, Inc, Stamford, CT.

Gimpel, H., Hosseini, S., Huber, R., Probst, L., Röglinger, M. and Faisst, U. (2018), "Structuring Digital Transformation. A Framework of Action Fields and its Application at ZEISS", Journal of Information Technology Theory and Application.

Gomes, J. and Romão, M. (2016), "Improving Project Success. A Case Study Using Benefits and Project Management”, Procedia Computer Science, Vol. 100, pp. 489-497.

Greener, S. (2008), Business research methods, Ventus Publishing ApS, London.

Grisold, T., Gross, S., Röglinger, M., Stelzl, K. and Vom Brocke, J. (2019), "Exploring Explorative BPM - Setting the Ground for Future Research”, in Hildebrandt, T., van Dongen, B.F., Röglinger, M. and Mendling, J. (Eds.), Business Process Management, Lecture Notes in Computer Science, Vol. 11675, Springer International Publishing, Cham, pp. 23-31.

Guimaraes, T. (1997), "Empirically testing the antecedents of BPR success", International Journal of Production Economics, Vol. 50 2-3, pp. 199-210. 
Haddad, A., Ameen, A.A. and Mukred, M. (2018), “The Impact of Intention of Use on the Success of Big Data Adoption via Organization Readiness Factor”, International Journal of Management and Human Science No. 2, pp. 43-51.

Heidt, M., Gerlach, J.P. and Buxmann, P. (2019), "Investigating the Security Divide between SME and Large Companies: How SME Characteristics Influence Organizational IT Security Investments”, Information Systems Frontiers, Vol. 21 No. 6, pp. 1285-1305.

Holotuik, F. and Beimborn, D. (2017), "Critical Success Factors of Digital Business Strategy", in 13th International Conference on Wirtschaftsinformatik, Vol. 13, pp. 991-1005.

Huber, R.X.R., Püschel, L.C. and Röglinger, M. (2019), “Capturing smart service systems: Development of a domain-specific modelling language”, Information Systems Journal, Vol. 29 No. 6, pp. 1207-1255.

Hughes, D.L., Rana, N.P. and Simintiras, A.C. (2017), "The changing landscape of IS project failure. An examination of the key factors", Journal of Enterprise Information Management, Vol. 30 No. 1, pp. 142-165.

Ika, L.A. (2009), "Project success as a topic in project management journals", Project Management Journal, Vol. 40 No. 4, pp. 6-19.

Irvine, R. and Hall, H. (2015), "Factors, frameworks and theory: a review of the information systems literature on success factors in project management”, Information Research, Vol. 20 No. 3.

Jugdev, K. and Müller, R. (2005), “A retrospective look at our evolving understanding of project success”, Project Management Journal, Vol. 36 No. 4, pp. 19-31.

Kerpedzhiev, G.D., König, U.M., Röglinger, M. and Rosemann, M. (2020), “An Exploration into Future Business Process Management Capabilities in View of Digitalization", Business \& Information Systems Engineering, Vol. 14, p. 33.

Kerzner, H. (2013), Project management: A systems approach to planning, scheduling, and controlling, 11th ed., John Wiley \& Sons, Inc., Hoboken, New Jersey.

Khan, F., Amyotte, P. and Adedigba, S. (2021), "Process safety concerns in process system digitalization", Education for Chemical Engineers, Vol. 34, pp. 33-46.

Kirsch, L.J. and Slaughter, S.A. (2013), "Managing the unmanageable: How IS research can contribute to the scholarship of cyber projects", Journal of the Association for Information Systems, Vol. 14 No. 4, pp. 198214.

Koleva, N. (2019), “An Empirical Study on Human Resoureces’ Attitude Towards Manufacturing Digitalization", in 2019 International Conference on Creative Business for Smart and Sustainable Growth (CREBUS), Sandanski, Bulgaria, 18.03.2019 - 21.03.2019, IEEE, pp. 1-5.

Kreuzer, T., Röglinger, M. and Rupprecht, L. (2020), “Customer-centric prioritization of process improvement projects", Decision Support Systems, Vol. 133, p. 113286.

Krippendorff, K. (2013), Content analysis: An introduction to its methodology, Sage, Los Angeles, London, New Delhi, Singapore.

Lam, S.L., Cheung, R., Wong, S. and Chan, E.S. (2013), “A survey study of critical success factors in information system project management", in International Conference on Internet Studies.

Lange, M., Mendling, J. and Recker, J. (2016), “An empirical analysis of the factors and measures of Enterprise Architecture Management success”, European Journal of Information Systems, Vol. 25 No. 5, pp. 411-431.

Legner, C., Eymann, T., Hess, T., Matt, C., Böhmann, T., Drews, P., Mädche, A., Urbach, N. and Ahlemann, F. (2017), "Digitalization. Opportunity and Challenge for the Business and Information Systems Engineering Community”, Business \& Information Systems Engineering, Vol. 59 No. 4, pp. 301-308. 
Lehnert, M., Linhart, A. and Röglinger, M. (2016), "Value-based process project portfolio management: integrated planning of BPM capability development and process improvement", Business Research, Vol. 9 No. 2, pp. 377-419.

Levi, M., Kleindorfer, P.R. and Wu, D. (2003), “Codifiability, Relationship-Specific Information Technology Investment, and Optimal Contracting”, Journal of Management Information Systems, Vol. 20 No. 2, pp. $77-$ 98.

Limam Mansar, S., Reijers, H.A. and Ounnar, F. (2009), "Development of a decision-making strategy to improve the efficiency of BPR", Expert Systems with Applications, Vol. 36 No. 2, pp. 3248-3262.

Loebbecke, C. and Picot, A. (2015), "Reflections on societal and business model transformation arising from digitization and big data analytics. A research agenda", The Journal of Strategic Information Systems, Vol. 24 No. 3, pp. 149-157.

Majchrzak, A., Markus, M.L. and Wareham, J. (2016), "Designing for digital transformation. Lessons for information systems research from the study of ICT and societal challenges", MIS Quarterly, Vol. 40 No. 2, pp. $267-277$.

Malinova, M., Hribar, B. and Mendling, J. (2014), “A Framework for Assessing BPM Success”, in Proceedings of the 22nd European Conference on Information Systems, pp. 1-15.

Marshall, B., Cardon, P., Poddar, A. and Fontenot, R. (2013), "Does Sample Size Matter in Qualitative Research?: A Review of Qualitative Interviews in is Research", Journal of Computer Information Systems, Vol. 54 No. 1, pp. 11-22.

Martinsuo, M., Korhonen, T. and Laine, T. (2014), "Identifying, framing and managing uncertainties in project portfolios", International Journal of Project Management, Vol. 32 No. 5, pp. 732-746.

Matt, C., Hess, T. and Benlian, A. (2015), "Digital transformation strategies", Business \& Information Systems Engineering, Vol. 57 No. 5, pp. 339-343.

McLean, R. and Antony, J. (2014), "Why continuous improvement initiatives fail in manufacturing environments? A systematic review of the evidence", International Journal of Productivity and Performance Management, Vol. 63 No. 3, pp. 370-376.

McLeod, L., Doolin, B. and MacDonell, S.G. (2012), “A Perspective-Based Understanding of Project Success”, Project Management Journal, Vol. 43 No. 5, pp. 68-86.

Mendling, J., Pentland, B. and Recker, J. (2020), "Building a Complementary Agenda for Business Process Management and Digital Innovation”, European Journal of Information Systems, pp. 1-25.

Morana, S., Kroenung, J., Maedche, A. and Schacht, S. (2019), "Designing Process Guidance Systems”, Journal of the Association for Information Systems, pp. 499-535.

Myers, M.D. and Newman, M. (2007), "The qualitative interview in IS research. Examining the craft”, Information and Organization, Vol. 17 No. 1, pp. 2-26.

Pankratz, O. and Loebbecke, C. (2011), "Project managers' perception of IS project success factors - a repertory grid investigation", in Proceedings of the 19th European Conference on Information Systems, pp. 1-13.

Paper, D. and Chang, R.-D. (2005), "The state of business process reengineering. A search for success factors", Total Quality Management \& Business Excellence, Vol. 16 No. 1, pp. 121-133.

Pellegrinelli, S., Murray-Webster, R. and Turner, N. (2015), "Facilitating organizational ambidexterity through the complementary use of projects and programs", International Journal of Project Management, Vol. 33 No. 1, pp. 153-164.

Peralta, C.F., Lopes, P.N., Gilson, L.L., Lourenço, P.R. and Pais, L. (2015), "Innovation processes and team effectiveness: The role of goal clarity and commitment, and team affective tone", Journal of Occupational and Organizational Psychology, Vol. 88 No. 1, pp. 80-107. 
Petter, S., DeLone, W. and McLean, E.R. (2013), "Information systems success: The quest for the independent variables", Journal of Management Information Systems, Vol. 29 No. 4, pp. 7-62.

Porter, M.E. and Heppelmann, J.E. (2015), "How smart, connected products are transforming companies", Harvard Business Review, Vol. 93 No. 10, pp. 96-114.

Ram, J., Corkindale, D. and Wu, M.-L. (2013), "Implementation critical success factors (CSFs) for ERP. Do they contribute to implementation success and post-implementation performance?", International Journal of Production Economics, Vol. 144 No. 1, pp. 157-174.

Raziq, M.M., Borini, F.M., Malik, O.F., Ahmad, M. and Shabaz, M. (2018), “Leadership styles, goal clarity, and project success", Leadership \& Organization Development Journal, Vol. 39 No. 2, pp. 309-323.

Recker, J. and Mendling, J. (2016), "The State of the Art of Business Process Management Research as Published in the BPM Conference”, Business \& Information Systems Engineering, Vol. 58 No. 1, pp. 55-72.

Reif, J.A., Kugler, K.G. and Brodbeck, F.C. (2019), “The regulatory power of standardized business processes”, Business Process Management Journal, Vol. 25 No. 5, pp. 1126-1144.

Reijers, H.A. and Limam Mansar, S. (2005), "Best practices in business process redesign. An overview and qualitative evaluation of successful redesign heuristics”, Omega, Vol. 33 No. 4, pp. 283-306.

Remus, U. (2007), “Critical success factors for implementing enterprise portals”, Business Process Management Journal, Vol. 13 No. 4, pp. 538-552.

Ren, J., Yusuf, Y. and Burns, N. (2005), “Agile partner selection: a hierarchical model and empirical investigation”, International Journal of Information and Systems Sciences, Vol. 1 No. 1, pp. 1-22.

Rodriguez, R., Molina-Castillo, F.-J. and Svensson, G. (2020a), "Enterprise resource planning and business model innovation: process, evolution and outcome", European Journal of Innovation Management, Vol. 23 No. 4, pp. 728-752.

Rodríguez, R., Molina-Castillo, F.-J. and Svensson, G. (2020b), “The mediating role of organizational complexity between enterprise resource planning and business model innovation”, Industrial Marketing Management, Vol. 84, pp. 328-341.

Rosemann, M. (2006), "Potential pitfalls of process modeling: part B”, Business Process Management Journal, Vol. 12 No. 3, pp. 377-384.

Rosemann, M. (2020), “Explorative Process Design Patterns”, in Fahland, D., Ghidini, C., Becker, J. and Dumas, M. (Eds.), Business Process Management, Lecture Notes in Computer Science, Vol. 12168, Springer International Publishing, Cham, pp. 349-367.

Rosemann, M. and Vom Brocke, J. (2015), “The Six Core Elements of Business Process Management”, in Vom Brocke, J. and Rosemann, M. (Eds.), Handbook on Business Process Management 1: Introduction, Methods, and Information Systems, International Handbooks on Information Systems, 2nd ed. 2015, Springer Berlin Heidelberg, Berlin, Heidelberg, s.1., pp. 105-122.

Sajid, Z., Khan, M.K., Rahnama, A., Moghaddam, F.S., Vardhan, K. and Kalani, R. (2021), “Computational Fluid Dynamics (CFD) Modeling and Analysis of Hydrocarbon Vapor Cloud Explosions (VCEs) in Amuay Refinery and Jaipur Plant Using FLACS”, Processes, Vol. 9 No. 6, p. 960.

Sarker, S., Xiao, X. and Beaulieu, T. (2013), "Qualitative Studies in Information Systems: A Critical Review and Some Guiding Principles”, MIS Quarterly: Management Information Systems, Vol. 37, pp. iii-xviii.

Schmiedel, T., Recker, J. and Vom Brocke, J. (2020), “The relation between BPM culture, BPM methods, and process performance: Evidence from quantitative field studies", Information \& management, Vol. 57 No. 2 , p. 103175.

Shenhar, A.J., Tishler, A., Dvir, D., Lipovetsky, S. and Lechler, T. (2002), "Refining the search for project success factors. A multivariate, typological approach”, $R$ and D Management, Vol. 32 No. 2, pp. 111-126. 
Siegel, N.G. and Madni, A.M. (2014), "The Digital Battlefield. A Behind-the-Scenes Look from a Systems Perspective", Procedia Computer Science, Vol. 28, pp. 799-808.

Siha, S.M. and Saad, G.H. (2008), "Business process improvement. Empirical assessment and extensions", Business Process Management Journal, Vol. 14 No. 6, pp. 778-802.

Soluk, J. and Kammerlander, N. (2021), "Digital transformation in family-owned Mittelstand firms: A dynamic capabilities perspective", European Journal of Information Systems, Vol. 16 No. 2, pp. 1-36.

Trkman, P. (2010), “The critical success factors of business process management", International Journal of Information Management, Vol. 30 No. 2, pp. 125-134.

Umble, E.J., Haft, R.R. and Umble, M. (2003), "Enterprise resource planning. Implementation procedures and critical success factors", European Journal of Operational Research, Vol. 146 No. 2, pp. 241-257.

van der Aalst, W.M.P. (2013), "Business Process Management. A Comprehensive Survey", ISRN Software Engineering, Vol. 2013 No. 1, pp. 1-37.

Varajão, J. and Trigo, A. (2016), "Evaluation of IS project success in InfSysMakers. an exploratory case study", Digital Innovation at the Crossroads, Proceedings of the International Conference on Information Systems, pp. $1-10$.

Vial, G. (2019), "Understanding digital transformation: A review and a research agenda", The Journal of Strategic Information Systems, Vol. 28 No. 2, pp. 118-144.

Vom Brocke, J., Denner, M.-S., Schmiedel, T., Stelzl, K., Röglinger, M. and Wehking, C. (2020), "ContextAware Business Process Management: Method Assessment and Selection”, Business \& Information Systems Engineering, Vol. 62.

Vom Brocke, J., Simons, A., Riemer, K., Niehaves, B., Plattfaut, R. and Cleven, A. (2015), "Standing on the Shoulders of Giants. Challenges and Recommendations of Literature Search in Information Systems Research", Communications of the Association for Information Systems, Vol. 37 No. 1, pp. 205-224.

Vom Brocke, J., Zelt, S. and Schmiedel, T. (2016), "On the role of context in business process management", International Journal of Information Management, Vol. 36 No. 3, pp. 486-495.

Webster, J. and Watson, R. (2002), “Analyzing the Past to Prepare for the Future: Writing a Literature Review", MIS Quarterly, Vol. 26.

Wessel, L., Baiyere, A., Ologeanu-Taddei, R., Cha, J. and Blegind Jensen, T. (2021), "Unpacking the Difference Between Digital Transformation and IT-Enabled Organizational Transformation", Journal of the Association for Information Systems, Vol. 22 No. 1, pp. 102-129.

Wolfswinkel, J.F., Furtmueller, E. and Wilderom, C.P.M. (2013), "Using grounded theory as a method for rigorously reviewing literature", European Journal of Information Systems, Vol. 22 No. 1, pp. 45-55.

Yayavaram, S., Srivastava, M.K. and Sarkar, M.B. (2018), "Role of search for domain knowledge and architectural knowledge in alliance partner selection", Strategic management journal, Vol. 39 No. 8, pp. 2277-2302.

Yoo, Y., Henfridsson, O. and Lyytinen, K. (2010), "Research Commentary - The New Organizing Logic of Digital Innovation. An Agenda for Information Systems Research", Information systems research, Vol. 21 No.

4, pp. 724-735. 


\section{Appendix A: Success Codes extracted from the Literature}

This table shows the total number of mentions per success factor received in a respective study retrieved in the structured database search. Used abbreviations are:

- SF: Coding of the candidate PDP Success Factor

- SFC: Coding of the category of PDP Success Factors

- RF: Research field

- Digit*: all fields related to Digitalization

- BPI: Business Process Improvement

- BPR: Business Process Reengineering

- PM: Project Management

- PPM: Project Portfolio Management

\begin{tabular}{|c|c|c|c|c|c|c|c|c|c|c|c|c|c|c|c|c|c|c|c|c|c|c|c|c|c|c|c|c|c|c|c|c|}
\hline & & 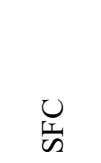 & $\frac{\mathscr{O}}{\stackrel{\Xi}{\Xi}}$ & $\frac{\mathscr{\Xi}}{\stackrel{\Xi}{\Xi}}$ & $\frac{\mathscr{O}}{\stackrel{\Xi}{\Xi}}$ & $\frac{\mathscr{\Xi}}{\Xi}$ & 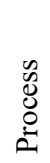 & $\begin{array}{l}\tilde{u} \\
0 \\
0 \\
0 \\
0\end{array}$ & 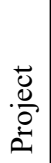 & $\mid \begin{array}{c}\overrightarrow{0} \\
\cdot \frac{0}{O} \\
\dot{0} \\
0\end{array}$ & $\begin{array}{c}\vec{U} \\
\frac{\tilde{U}}{O} \\
\dot{D}\end{array}$ & $\begin{array}{l}\vec{u} \\
\stackrel{\tilde{\omega}}{O} \\
\stackrel{2}{2}\end{array}$ & $\begin{array}{c}\vec{U} \\
. \overrightarrow{0} \\
\stackrel{0}{D} \\
\end{array}$ & 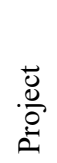 & 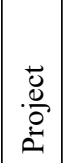 & $\mid \begin{array}{c}\vec{U} \\
\frac{\tilde{U}}{O} \\
\overrightarrow{0}\end{array}$ & $\left|\begin{array}{c}0 \\
\frac{0}{2} \\
0 \\
0 \\
2\end{array}\right|$ & $\begin{array}{c}0 \\
\frac{0}{2} \\
0 \\
0\end{array}$ & $\begin{array}{l}\frac{0}{2} \\
\frac{0}{0} \\
2\end{array}$ & $\begin{array}{l}0 \\
\frac{0}{2} \\
0 \\
0\end{array}$ & $\mid \begin{array}{c}\frac{0}{2} \\
0 \\
0 \\
0\end{array}$ & $\left|\begin{array}{c}0 \\
\frac{0}{2} \\
0 \\
0 \\
2\end{array}\right|$ & $\frac{0}{2}$ & $\mid \begin{array}{l}0 \\
0 \\
0 \\
0 \\
0\end{array}$ & 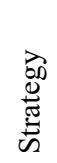 & 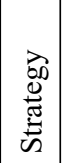 & 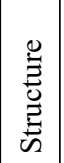 & 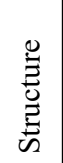 & $\begin{array}{c}\overrightarrow{0} \\
0 \\
0 \\
0 \\
0 \\
0 \\
e \\
\oplus \\
\oplus\end{array}$ & 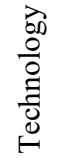 & 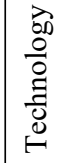 & 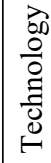 \\
\hline Source & $\mathrm{RF}$ & $\sqrt[L]{\omega}$ & 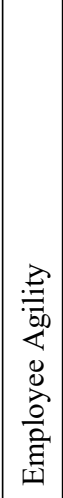 & 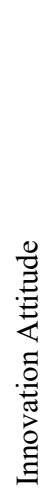 & 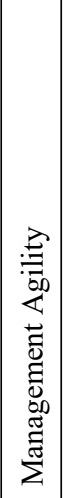 & 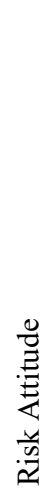 & 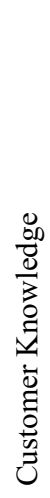 & 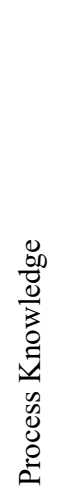 & 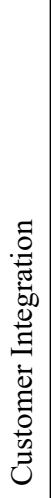 & 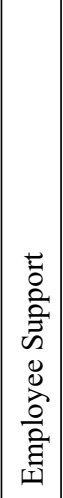 & 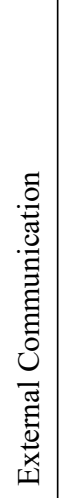 & 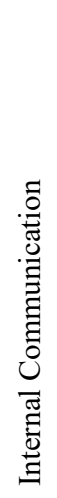 & 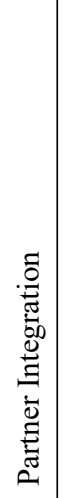 & 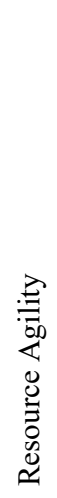 & 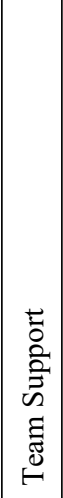 & 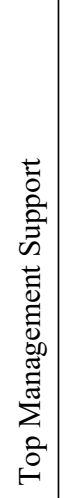 & 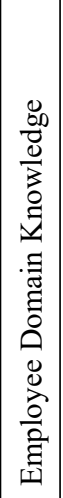 & 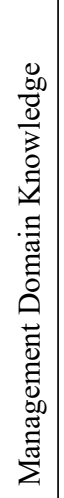 & 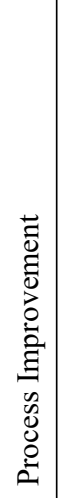 & 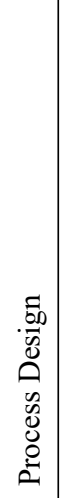 & 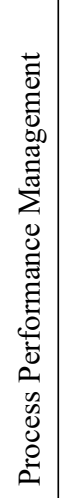 & 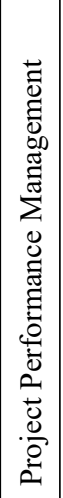 & 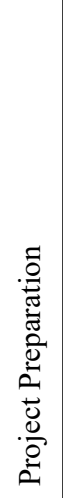 & 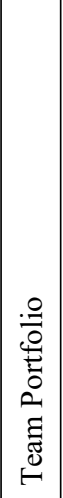 & 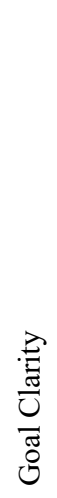 & 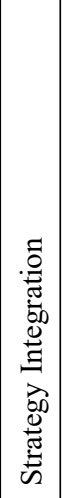 & 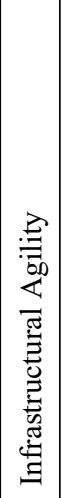 & 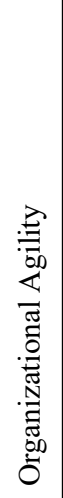 & 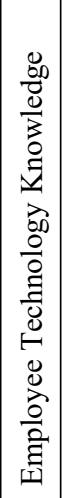 & 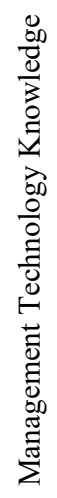 & 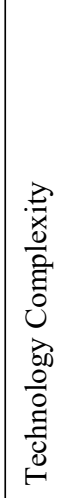 & 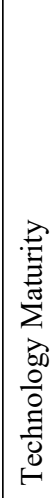 \\
\hline Abollado et al. (2017) & Digit* & $\sum=23$ & 1 & & 1 & & & 3 & 1 & 1 & & 2 & 1 & & & 2 & & & & & 3 & & 2 & & & & 5 & & & & & 1 \\
\hline Holotuik and Beimborn (2017) & Digit* & $\sum=41$ & & 9 & 2 & 2 & 1 & & 3 & & & & 2 & 1 & & & & 3 & 2 & 1 & & & & 3 & & 3 & 5 & 4 & & & & \\
\hline Siegel and Madni (2014) & Digit* & $\sum=6$ & & 1 & & 2 & & & & & & & & & & 1 & & & & & & 1 & 1 & & & & & & & & & \\
\hline Antony et al. (2012) & BPI & $\sum=16$ & & & & & & & 2 & & & 3 & & 1 & 1 & 1 & & 1 & & & & 1 & 1 & & 3 & & 1 & 1 & & & & \\
\hline Malinova et al. (2014) & BPI & $\sum=22$ & & 1 & 1 & 1 & 2 & 5 & 1 & & & 3 & & & & & 1 & & 5 & & 1 & & & & & & 1 & & & & & \\
\hline
\end{tabular}




\begin{tabular}{|c|c|c|c|c|c|c|c|c|c|c|c|c|c|c|c|c|c|c|c|c|c|c|c|c|c|c|c|c|c|c|c|c|}
\hline McLean and Antony (2014) & BPI & $\sum=8$ & & & & & & & & 1 & & 2 & & & & 1 & & 1 & & & & & 1 & & 1 & & & 1 & & & & \\
\hline Siha and Saad (2008) & BPI & $\sum=53$ & & 1 & & & 2 & 2 & 1 & 2 & & 4 & 2 & & & 4 & & 3 & 6 & 3 & 2 & & 1 & 3 & 6 & 6 & 1 & 3 & & & & 1 \\
\hline Abdolvand et al. (2008) & BPR & $\sum=24$ & & 2 & & 1 & & 1 & & 1 & & 3 & & & 1 & & & 2 & & & & 1 & & 1 & 2 & & & 6 & & & & 3 \\
\hline Al-Mashari and Zairi (1999) & BPR & $\sum=51$ & & 5 & & & & & & 2 & 3 & 5 & & 4 & 1 & 4 & 2 & 1 & 4 & & & & 4 & 3 & 2 & 3 & 4 & 2 & & 1 & & 1 \\
\hline Dezdar (2012) & $\mathrm{BPR}$ & $\sum=5$ & & & & & & & & & & 2 & 1 & & & 1 & & 1 & & & & & & & & & & & & & & \\
\hline Dezdar and Ainin (2011) & BPR & $\sum=11$ & & & & & & & & & & & 2 & & & & 1 & 2 & & & & 1 & 2 & 1 & 1 & & & & 1 & & & \\
\hline Guimaraes (1997) & BPR & $\Sigma=32$ & & 2 & & & 2 & & 1 & & 1 & 4 & 1 & & 2 & 2 & & 1 & 5 & 3 & & & 1 & 1 & 3 & 1 & 1 & 1 & & & & \\
\hline Paper and Chang (2005) & BPR & $\sum=37$ & 1 & 2 & 1 & 2 & & 2 & 2 & 2 & & 6 & & 1 & 2 & 3 & 2 & 1 & & & 1 & & 1 & 2 & & 2 & 1 & 1 & 2 & & & \\
\hline Umble et al. (2003) & $\mathrm{BPR}$ & $\sum=15$ & & 1 & & & & & & 1 & & 1 & & & & 2 & & 1 & & & 1 & & 2 & 1 & 2 & & 2 & & 1 & & & \\
\hline Beringer et al. (2013) & PPM & $\sum=7$ & & & & & & & & & & & & & 1 & 3 & & & & & & & & 2 & & 1 & & & & & & \\
\hline Costantino et al. (2015) & PPM & $\sum=16$ & & & & 3 & 1 & & 1 & & 1 & & & 1 & & 1 & & 1 & & & & 1 & 2 & 2 & & & & 1 & 1 & & & \\
\hline Frey and Buxmann (2012) & PPM & $\sum=10$ & & & & 1 & & & & & & & & 1 & & 1 & & & & & & 1 & 3 & & 1 & 2 & & & & & & \\
\hline Allen et al. (2014) & $\mathrm{PM}$ & $\sum=12$ & & & & & & 1 & 1 & & & 1 & & & 1 & & & & & & 1 & & 2 & & 1 & & & 4 & & & & \\
\hline Avital (2003) & PM & $\sum=40$ & & 2 & & & 1 & 2 & 2 & & 1 & 9 & & 1 & 2 & 2 & 3 & 1 & & & & & & 3 & 4 & 1 & 1 & 4 & & & & 1 \\
\hline Bai and Sarkis (2013) & PM & $\sum=12$ & & & & & & & 1 & & & 1 & & & & 2 & & 2 & & & 1 & & & & & 1 & 1 & 3 & & & & \\
\hline Besteiro et al. (2015) & PM & $\sum=23$ & & & & 1 & & & & 2 & 1 & 3 & & 2 & & & & 2 & & & & & 5 & 1 & 4 & & & 2 & & & & \\
\hline Bradley (2005) & PM & $\sum=5$ & & & & & & & & & 1 & 1 & & & & 1 & & & & & & & & 1 & & & & & & 1 & & \\
\hline Clarke (1999) & PM & $\sum=12$ & & & 1 & & & 1 & & & & 4 & & & & & & 1 & & & & 1 & 2 & & 2 & & & & & & & \\
\hline Cooke-Davies (2002) & PM & $\sum=12$ & & 1 & & 3 & & & & & & 2 & & & & & & & & & 1 & 1 & 1 & & & 1 & & 2 & & & & \\
\hline Dvir et al. (1998) & PM & $\sum=97$ & & 1 & 1 & 1 & 1 & 2 & 3 & & 3 & 8 & & 2 & 3 & 3 & 2 & 8 & 3 & 3 & 6 & 7 & 13 & 5 & 4 & & 4 & 5 & 2 & 1 & 2 & 4 \\
\hline Fowler and Horan (2007) & PM & $\sum=12$ & & & & & 1 & & 2 & 2 & & 1 & & & & 2 & 2 & 2 & & & & & & & & & & & & & & \\
\hline Gomes and Romão (2016) & PM & $\sum=8$ & & & & 1 & 2 & & & & & & & 2 & 1 & 1 & & & & & & 1 & & & & & & & & & & \\
\hline Irvine and Hall (2015) & PM & $\sum=47$ & 2 & 1 & & 1 & & & 2 & 3 & 1 & 2 & & 1 & 1 & 2 & & 5 & & & 1 & 2 & 10 & 6 & 6 & & & & & & & 1 \\
\hline Hughes et al. (2017) & PM & $\sum=67$ & & 1 & 3 & 3 & 1 & 2 & 3 & 2 & & 5 & 1 & 5 & 4 & 4 & & 8 & 1 & & & 3 & 12 & 1 & 1 & & & 3 & & 2 & 1 & 1 \\
\hline Ika (2009) & PM & $\sum=2$ & & & & & & & & & & & & & & & & 1 & & & & & 1 & & & & & & & & & \\
\hline Jugdev and Müller (2005) & $\mathrm{PM}$ & $\sum=58$ & & 1 & 3 & & 3 & 1 & 2 & & 4 & 3 & 2 & & & 5 & 1 & 4 & 1 & & 1 & 1 & 5 & 3 & 6 & 6 & 1 & 3 & & & 1 & 1 \\
\hline Kirsch and Slaughter (2013) & PM & $\sum=15$ & & & & & 1 & & & & & 1 & & & & & & 2 & & & 2 & & 2 & 2 & 1 & & 1 & 2 & & & & 1 \\
\hline Lam et al. (2013) & PM & $\sum=58$ & 1 & & 1 & 1 & 1 & & 6 & 1 & & 4 & & 1 & 2 & 4 & 1 & 4 & 1 & & & 1 & 7 & 4 & 4 & & 1 & 10 & & & 2 & 1 \\
\hline $\begin{array}{l}\text { Pankratz and Loebbecke } \\
(2011)\end{array}$ & PM & $\Sigma=58$ & 1 & & & 2 & & & 4 & & & 1 & 1 & & 3 & 5 & 1 & 6 & 1 & & 4 & 5 & 6 & 3 & 6 & 2 & & 5 & & & 2 & \\
\hline Ram et al. (2013) & PM & $\sum=31$ & & 1 & & & & & 1 & & 3 & 5 & 3 & & & 2 & & 2 & 2 & & 1 & 1 & 2 & 2 & 2 & 1 & 1 & 1 & 1 & & & \\
\hline
\end{tabular}


Remus (2007)

Shenhar et al. (2002)

Varajão and Trigo (2016)

Rodríguez et al. (2020b)

\begin{tabular}{|c|c|c|c|c|c|c|c|c|c|c|c|c|c|c|c|c|c|c|c|c|c|c|c|c|c|c|c|c|c|c|c|} 
PM & $\sum=42$ & 1 & 2 & & & 1 & & & & 1 & 4 & 3 & 2 & & 3 & & 2 & 3 & & & 1 & 6 & 3 & 3 & 2 & 2 & 1 & 1 & & & 1 \\
\hline PM & $\sum=18$ & & & & & & 2 & 1 & & & & & 1 & & & & 2 & & 1 & 1 & 2 & 2 & 1 & 1 & & & 4 & & & \\
\hline PM & $\sum=16$ & & & & 4 & & 3 & & & & & 1 & 1 & & & & & & & 1 & 3 & & 1 & 2 & & & & & \\
\hline BPI & $\sum=35$ & & & & & & & & & & & & & & & & & & & & & & & 1 & & & 1 & & \\
\hline BPR & $\sum=66$ & & & & & & & & & & & & & & & & & & & & & & & & 1 & 1 & & & 1 & \\
\hline
\end{tabular}




\section{Appendix B: List of Success Codes and respective Coding}

Two co-authors coded the success codes by assigning them to previously built success factor constructs. Then, an external coder assigned the success codes to the constructs as well. This table shows all extracted success codes numbered consecutively and coded as further explicated in the manuscript. Used abbreviations are:

- SC: Success Code

- SF: Coding of the candidate PDP Success Factor

- SCF: Coding of the Category of PDP Success Factors

- AX: Co-Author X

- EC: External Coder

- RF: Research Field

- Digit*: all fields related to Digitalization

- BPI: Business Process Improvement

- BPR: Business Process Reengineering

- PM: Project Management

- PPM: Project Portfolio Management

- SF number: 1=Employee Agility, 2=Innovation Attitude, 3=Management Agility,4=Risk Attitude, 5=Customer Knowledge, 6=Process Knowledge, 7=Customer Integration, 8=Employee Support, 9=External Communication, 10=Internal Communication, 11=Partner Integration, 12=Resource Agility, 13=Team Support, 14=Top Management Support, 15=Employee Domain Knowledge, 16=Management Domain Knowledge, 17=Process Improvement, 18=Process Design, 19=Process Performance Management, 20=Project Performance Management, 21=Project Preparation, 22=Team Portfolio, 23=Goal Clarity, 24=Strategy Integration, 25=Infrastructural Agility, 26=Organizational Agility, 27=Employee Technology Knowledge, 28=Management Technology Knowledge, 29=Technology Complexity, 30=Technology Maturity.

- SFC number: $1=$ Culture, $2=$ Process, $3=$ Project, $4=$ =People, $5=$ Strategy, $6=$ Structure, $7=$ Technology.

\begin{tabular}{|c|l|c|c|c|c|c|c|c|l|c|c|c|}
\hline $\begin{array}{c}\text { SC } \\
\text { No. }\end{array}$ & \multicolumn{1}{|c|}{ SC } & A1 & A1 & A2 & A2 & EC & EC & & Source & Year & $\begin{array}{l}\text { Success/ } \\
\text { Failure }\end{array}$ & $\begin{array}{l}\text { Explicit/ } \\
\text { Implicit }\end{array}$ \\
\hline 1 & $\begin{array}{l}\text { Risk management - Effective- } \\
\text { ness }\end{array}$ & 1 & 5 & 1 & 5 & 1 & 5 & PM & $\begin{array}{l}\text { Irvine } \\
\text { and Hall }\end{array}$ & 2015 & success & explicit \\
\hline 2 & Project team dynamics & 1 & 1 & 1 & 1 & 1 & 1 & PM & $\begin{array}{l}\text { Irvine } \\
\text { and Hall }\end{array}$ & 2015 & success & explicit \\
\hline 3 & $\begin{array}{l}\text { Incremental organizational } \\
\text { change }\end{array}$ & 1 & 2 & 1 & 2 & 1 & 2 & PM & $\begin{array}{l}\text { Irvine } \\
\text { and Hall }\end{array}$ & 2015 & failure & explicit \\
\hline 4 & $\begin{array}{l}\text { Staff turnover } \\
\text { ence }\end{array}$ & 1 & 1 & 1 & 1 & 3 & 9 & PM & $\begin{array}{l}\text { Irvine } \\
\text { and Hall }\end{array}$ & 2015 & failure & explicit \\
\hline 5 & Project team - Competence & 4 & 24 & 4 & 24 & 4 & 24 & PM & $\begin{array}{l}\text { Irvine } \\
\text { and Hall }\end{array}$ & 2015 & success & explicit \\
\hline 7 & $\begin{array}{l}\text { Client/host organization - Staff } \\
\text { turnover }\end{array}$ & 4 & 24 & 4 & 24 & 4 & 24 & PM & $\begin{array}{l}\text { Irvine } \\
\text { and Hall }\end{array}$ & 2015 & success & explicit \\
\hline 8 & Project team - Competence/fit & 4 & 24 & 4 & 24 & 4 & 24 & PM & $\begin{array}{l}\text { Irvine } \\
\text { and Hall }\end{array}$ & 2015 & success & explicit \\
\hline 9 & $\begin{array}{l}\text { Project team - Compe- } \\
\text { tence/technical }\end{array}$ & 4 & 24 & 4 & 24 & 4 & 24 & PM & $\begin{array}{l}\text { Irvine } \\
\text { and Hall }\end{array}$ & 2015 & success & explicit \\
\hline 10 & Project team - Experience & 4 & 24 & 4 & 24 & 4 & 24 & PM & $\begin{array}{l}\text { Irvine } \\
\text { and Hall }\end{array}$ & 2015 & success & explicit \\
\hline 11 & $\begin{array}{l}\text { Project planning - Effective- } \\
\text { ness }\end{array}$ & 4 & 23 & 4 & 23 & 4 & 23 & PM & $\begin{array}{l}\text { Irvine } \\
\text { and Hall }\end{array}$ & 2015 & success & explicit \\
\hline 12 & $\begin{array}{l}\text { Project management - Effec- } \\
\text { tiveness }\end{array}$ & 4 & 17 & 4 & 17 & 4 & 17 & PM & $\begin{array}{l}\text { Irvine } \\
\text { and Hall }\end{array}$ & 2015 & success & explicit \\
\hline
\end{tabular}




\begin{tabular}{|c|c|c|c|c|c|c|c|c|c|c|c|c|}
\hline 13 & Change control - Effectiveness & 4 & 21 & 4 & 21 & 1 & 2 & $\mathrm{PM}$ & $\begin{array}{l}\text { Irvine } \\
\text { and Hall }\end{array}$ & 2015 & success & explicit \\
\hline 14 & Project control - Effectiveness & 4 & 22 & 4 & 22 & 4 & 22 & $\mathrm{PM}$ & $\begin{array}{l}\text { Irvine } \\
\text { and Hall }\end{array}$ & 2015 & success & explicit \\
\hline 15 & $\begin{array}{l}\text { Project monitoring - Effective- } \\
\text { ness }\end{array}$ & 4 & 22 & 4 & 22 & 4 & 22 & $\mathrm{PM}$ & $\begin{array}{l}\text { Irvine } \\
\text { and Hall }\end{array}$ & 2015 & success & explicit \\
\hline 16 & Project - Size & 4 & 23 & 4 & 23 & 4 & 23 & $\mathrm{PM}$ & $\begin{array}{l}\text { Irvine } \\
\text { and Hall }\end{array}$ & 2015 & success & explicit \\
\hline 17 & Estimating - Effectiveness & 4 & 23 & 4 & 23 & 4 & 23 & $\mathrm{PM}$ & $\begin{array}{l}\text { Irvine } \\
\text { and Hall }\end{array}$ & 2015 & success & explicit \\
\hline 18 & Project - Complexity & 4 & 23 & 4 & 23 & 4 & 23 & $\mathrm{PM}$ & $\begin{array}{l}\text { Irvine } \\
\text { and Hall }\end{array}$ & 2015 & success & explicit \\
\hline 19 & $\begin{array}{l}\text { Requirements - Fitness for } \\
\text { purpose }\end{array}$ & 4 & 23 & 4 & 23 & 4 & 23 & PM & $\begin{array}{l}\text { Irvine } \\
\text { and Hall }\end{array}$ & 2015 & success & explicit \\
\hline 20 & Estimates - Fitness for purpose & 4 & 23 & 4 & 23 & 4 & 23 & $\mathrm{PM}$ & $\begin{array}{l}\text { Irvine } \\
\text { and Hall }\end{array}$ & 2015 & success & explicit \\
\hline 21 & Requirements - Completeness & 4 & 23 & 4 & 23 & 4 & 23 & PM & $\begin{array}{l}\text { Irvine } \\
\text { and Hall }\end{array}$ & 2015 & success & explicit \\
\hline 22 & $\begin{array}{l}\text { Project manager's leadership } \\
\text { style }\end{array}$ & 4 & 17 & 4 & 24 & 4 & 17 & $\mathrm{PM}$ & $\begin{array}{l}\text { Irvine } \\
\text { and Hall }\end{array}$ & 2015 & success & explicit \\
\hline 23 & $\begin{array}{l}\text { Project manager's use of vi- } \\
\text { sion }\end{array}$ & 4 & 17 & 4 & 24 & 4 & 17 & PM & $\begin{array}{l}\text { Irvine } \\
\text { and Hall }\end{array}$ & 2015 & success & explicit \\
\hline 24 & Project planning practices & 4 & 23 & 4 & 23 & 4 & 23 & $\mathrm{PM}$ & $\begin{array}{l}\text { Irvine } \\
\text { and Hall }\end{array}$ & 2015 & success & explicit \\
\hline 25 & Quality of planning & 4 & 23 & 4 & 23 & 4 & 23 & $\mathrm{PM}$ & $\begin{array}{l}\text { Irvine } \\
\text { and Hall }\end{array}$ & 2015 & success & explicit \\
\hline 26 & Risk management & 4 & 17 & 1 & 5 & 1 & 5 & PM & $\begin{array}{l}\text { Irvine } \\
\text { and Hall }\end{array}$ & 2015 & success & explicit \\
\hline 27 & Project size & 4 & 23 & 4 & 23 & 4 & 23 & PM & $\begin{array}{l}\text { Irvine } \\
\text { and Hall }\end{array}$ & 2015 & failure & explicit \\
\hline 28 & $\begin{array}{l}\text { Developer input to project es- } \\
\text { timates }\end{array}$ & 4 & 24 & 4 & 24 & 3 & 10 & PM & $\begin{array}{l}\text { Irvine } \\
\text { and Hall }\end{array}$ & 2015 & success & explicit \\
\hline 29 & End users - involvement & 3 & 8 & 3 & 8 & 3 & 8 & PM & $\begin{array}{l}\text { Irvine } \\
\text { and Hall }\end{array}$ & 2015 & success & explicit \\
\hline 30 & Project board - Supportiveness & 3 & 15 & 3 & 15 & 3 & 15 & $\mathrm{PM}$ & $\begin{array}{l}\text { Irvine } \\
\text { and Hall }\end{array}$ & 2015 & success & explicit \\
\hline 31 & $\begin{array}{l}\text { Communication - Effective- } \\
\text { ness }\end{array}$ & 3 & 10 & 3 & 10 & 3 & 10 & $\mathrm{PM}$ & $\begin{array}{l}\text { Irvine } \\
\text { and Hall }\end{array}$ & 2015 & success & explicit \\
\hline 32 & $\begin{array}{l}\text { Common knowledge (end us- } \\
\text { ers and project team) }\end{array}$ & 3 & 11 & 3 & 11 & 3 & 10 & $\mathrm{PM}$ & $\begin{array}{l}\text { Irvine } \\
\text { and Hall }\end{array}$ & 2015 & success & explicit \\
\hline 33 & End user involvement & 3 & 8 & 3 & 8 & 3 & 8 & PM & $\begin{array}{l}\text { Irvine } \\
\text { and Hall }\end{array}$ & 2015 & success & explicit \\
\hline 34 & $\begin{array}{l}\text { Executive management sup- } \\
\text { port }\end{array}$ & 3 & 15 & 3 & 15 & 3 & 15 & PM & $\begin{array}{l}\text { Irvine } \\
\text { and Hall }\end{array}$ & 2015 & success & explicit \\
\hline 35 & Organizational support & 3 & 9 & 3 & 9 & 3 & 9 & $\mathrm{PM}$ & $\begin{array}{l}\text { Irvine } \\
\text { and Hall }\end{array}$ & 2015 & success & explicit \\
\hline 36 & Project commitment & 3 & 9 & 3 & 14 & 3 & 9 & PM & $\begin{array}{l}\text { Irvine } \\
\text { and Hall }\end{array}$ & 2015 & success & explicit \\
\hline 37 & Project communications & 3 & 11 & 3 & 11 & 3 & 11 & PM & $\begin{array}{l}\text { Irvine } \\
\text { and Hall }\end{array}$ & 2015 & success & explicit \\
\hline 38 & Project sponsorship & 3 & 13 & 3 & 13 & 3 & 8 & PM & $\begin{array}{l}\text { Irvine } \\
\text { and Hall }\end{array}$ & 2015 & success & explicit \\
\hline 39 & Project team motivation & 3 & 14 & 3 & 14 & 3 & 14 & PM & $\begin{array}{l}\text { Irvine } \\
\text { and Hall }\end{array}$ & 2015 & success & explicit \\
\hline 40 & $\begin{array}{l}\text { Supportive organizational en- } \\
\text { vironment }\end{array}$ & 3 & 9 & 3 & 9 & 6 & 28 & $\mathrm{PM}$ & $\begin{array}{l}\text { Irvine } \\
\text { and Hall }\end{array}$ & 2015 & success & explicit \\
\hline
\end{tabular}




\begin{tabular}{|c|c|c|c|c|c|c|c|c|c|c|c|c|}
\hline 41 & Requirements - Stability & 5 & 25 & 5 & 25 & 5 & 25 & PM & $\begin{array}{l}\text { Irvine } \\
\text { and Hall }\end{array}$ & 2015 & success & explicit \\
\hline 42 & Requirements - Clarity & 5 & 25 & 5 & 25 & 5 & 25 & PM & \begin{tabular}{|l} 
Irvine \\
and Hall
\end{tabular} & 2015 & success & explicit \\
\hline 43 & Project vision & 5 & 25 & 5 & 25 & 5 & 25 & PM & $\begin{array}{l}\text { Irvine } \\
\text { and Hall }\end{array}$ & 2015 & success & explicit \\
\hline 44 & Requirements engineering & 5 & 25 & 5 & 25 & 5 & 25 & PM & \begin{tabular}{|l|} 
Irvine \\
and Hall
\end{tabular} & 2015 & success & explicit \\
\hline 45 & Goal changes & 5 & 25 & 1 & 3 & 5 & 25 & PM & \begin{tabular}{|l}
$\begin{array}{l}\text { Irvine } \\
\text { and Hall }\end{array}$ \\
\end{tabular} & 2015 & failure & explicit \\
\hline 46 & Project volatility & 5 & 25 & 5 & 25 & 5 & 25 & PM & $\begin{array}{l}\text { Irvine } \\
\text { and Hall }\end{array}$ & 2015 & failure & explicit \\
\hline 47 & $\begin{array}{l}\text { Project deliverable/technology } \\
\text { - Maturity }\end{array}$ & 7 & 33 & 7 & 33 & 7 & 33 & PM & $\begin{array}{l}\text { Irvine } \\
\text { and Hall }\end{array}$ & 2015 & success & explicit \\
\hline 48 & $\begin{array}{l}\text { Agility to reallocate resources } \\
\text { and reorganize rapidly }\end{array}$ & 1 & 3 & 1 & 3 & 6 & 28 & Digit* & $\begin{array}{l}\text { Holotiuk } \\
\text { and Beimborn }\end{array}$ & 2017 & success & explicit \\
\hline 49 & $\begin{array}{l}\text { Change management for radi- } \\
\text { cal and rapid change }\end{array}$ & 1 & 2 & 1 & 2 & 1 & 2 & Digit* & $\begin{array}{l}\text { Holotiuk } \\
\text { and Beimborn }\end{array}$ & 2017 & success & explicit \\
\hline 50 & $\begin{array}{l}\text { Long-term orientation but } \\
\text { short, intense sprints to change }\end{array}$ & 1 & 2 & 1 & 2 & 1 & 2 & Digit* & $\begin{array}{l}\text { Holotiuk } \\
\text { and Beimborn }\end{array}$ & 2017 & success & xplicit \\
\hline 51 & $\begin{array}{l}\text { Create and foster digital mind- } \\
\text { set with a digital agenda }\end{array}$ & 1 & 2 & 1 & 2 & 1 & 2 & Digit* & $\begin{array}{l}\text { Holotiuk } \\
\text { and Beimborn }\end{array}$ & 2017 & success & explicit \\
\hline 52 & Accept failure & 1 & 5 & 1 & 5 & 1 & 5 & Digit* & $\begin{array}{l}\text { Holotiuk } \\
\text { and Beimborn }\end{array}$ & 2017 & success & explicit \\
\hline 53 & $\begin{array}{l}\text { Encourage new to grow suc- } \\
\text { cess }\end{array}$ & 1 & 2 & 1 & 2 & 1 & 2 & Digit* & $\begin{array}{l}\text { Holotiuk } \\
\text { and Beimborn }\end{array}$ & 2017 & success & explicit \\
\hline 54 & $\begin{array}{l}\text { Innovation and adaptive cul- } \\
\text { ture with evolvable goals }\end{array}$ & 1 & 2 & 1 & 2 & 1 & 2 & Digit* & $\begin{array}{l}\text { Holotiuk } \\
\text { and Beimborn }\end{array}$ & 2017 & success & explicit \\
\hline 55 & $\begin{array}{l}\text { Commitment to transfor- } \\
\text { mation in strategy and culture }\end{array}$ & 1 & 2 & 1 & 2 & 1 & 2 & Digit* & $\begin{array}{l}\text { Holotiuk } \\
\text { and Beimborn }\end{array}$ & 2017 & success & explicit \\
\hline 56 & $\begin{array}{l}\text { Rethinking of C-level roles } \\
(\mathrm{CDO}, \mathrm{CIO})\end{array}$ & 1 & 3 & 1 & 3 & 1 & 3 & Digit* & $\begin{array}{l}\text { Holotiuk } \\
\text { and Beimborn }\end{array}$ & 2017 & success & explicit \\
\hline 57 & $\begin{array}{l}\text { Capability to reinvent value } \\
\text { chain and to challenge status } \\
\text { quo }\end{array}$ & 1 & 2 & 1 & 2 & 1 & 2 & Digit* & $\begin{array}{l}\text { Holotiuk } \\
\text { and Beimborn }\end{array}$ & 2017 & success & explicit \\
\hline 58 & $\begin{array}{l}\text { Foster faster innovation / rapid } \\
\text { prototyping }\end{array}$ & 1 & 2 & 1 & 2 & 1 & 2 & Digit* & $\begin{array}{l}\text { Holotiuk } \\
\text { and Beimborn }\end{array}$ & 2017 & success & explicit \\
\hline 59 & $\begin{array}{l}\text { Look what is laying left and } \\
\text { right }\end{array}$ & 1 & 2 & 1 & 2 & 1 & 2 & Digit* & $\begin{array}{l}\text { Holotiuk } \\
\text { and Beimborn }\end{array}$ & 2017 & success & explicit \\
\hline 60 & Bold experimentation & 1 & 5 & 1 & 5 & 1 & 5 & Digit* & $\begin{array}{l}\text { Holotiuk } \\
\text { and Beimborn }\end{array}$ & 2017 & success & explicit \\
\hline 61 & $\begin{array}{l}\text { Analytics to customize and } \\
\text { create productsandservices }\end{array}$ & 4 & 19 & 4 & 19 & 4 & 19 & Digit* & $\begin{array}{l}\text { Holotiuk } \\
\text { and Beimborn }\end{array}$ & 2017 & success & explicit \\
\hline 62 & \begin{tabular}{|l} 
Digital skills, know-how, and \\
talent
\end{tabular} & 4 & 24 & 4 & 24 & 4 & 24 & Digit* & \begin{tabular}{|l} 
Holotiuk \\
and Beimborn
\end{tabular} & 2017 & success & explicit \\
\hline 63 & $\begin{array}{l}\text { Capability to design new busi- } \\
\text { ness models }\end{array}$ & 4 & 17 & 4 & 17 & 4 & 17 & Digit* & \begin{tabular}{|l}
$\begin{array}{l}\text { Holotiuk } \\
\text { and Beimborn }\end{array}$ \\
\end{tabular} & 2017 & success & explicit \\
\hline 64 & New assets and capabilities & 4 & 24 & 1 & 2 & 1 & 2 & Digit* & $\begin{array}{l}\text { Holotiuk } \\
\text { and Beimborn }\end{array}$ & 2017 & success & explicit \\
\hline 65 & $\begin{array}{l}\text { Leaders have to identify new } \\
\text { HR potentials }\end{array}$ & 4 & 17 & 4 & 17 & 4 & 17 & Digit* & $\begin{array}{l}\text { Holotiuk } \\
\text { and Beimborn }\end{array}$ & 2017 & success & explicit \\
\hline 66 & $\begin{array}{l}\text { Acquire, retain, and attract } \\
\text { new talents }\end{array}$ & 4 & 17 & 4 & 24 & 4 & 17 & Digit* & $\begin{array}{l}\text { Holotiuk } \\
\text { and Beimborn }\end{array}$ & 2017 & success & explicit \\
\hline 67 & $\begin{array}{l}\text { Tight feedback loops and aspi- } \\
\text { ration to improvements }\end{array}$ & 4 & 19 & 4 & 19 & 4 & 19 & Digit* & $\begin{array}{l}\text { Holotiuk } \\
\text { and Beimborn }\end{array}$ & 2017 & success & explicit \\
\hline 68 & $\begin{array}{l}\text { Data-driven and digitally auto- } \\
\text { mated process }\end{array}$ & 4 & 20 & 4 & 20 & 4 & 19 & Digit* & $\begin{array}{l}\text { Holotiuk } \\
\text { and Beimborn }\end{array}$ & 2017 & success & explicit \\
\hline
\end{tabular}




\begin{tabular}{|c|c|c|c|c|c|c|c|c|c|c|c|c|}
\hline 69 & $\begin{array}{l}\text { Blending human and digital } \\
\text { resources }\end{array}$ & 4 & 24 & 4 & 24 & 4 & 24 & Digit* & $\begin{array}{l}\text { Holotiuk } \\
\text { and Beimborn }\end{array}$ & 2017 & success & explicit \\
\hline 70 & $\begin{array}{l}\text { Outstanding customer experi- } \\
\text { ence and satisfaction }\end{array}$ & 2 & 6 & 2 & 6 & 2 & 6 & Digit* & $\begin{array}{l}\text { Holotiuk } \\
\text { and Beimborn }\end{array}$ & 2017 & success & explicit \\
\hline 71 & $\begin{array}{l}\text { Digitalization of customer in- } \\
\text { teraction and productsandser- } \\
\text { vices }\end{array}$ & 3 & 8 & 3 & 8 & 3 & 8 & Digit* & $\begin{array}{l}\text { Holotiuk } \\
\text { and Beimborn }\end{array}$ & 2017 & success & explicit \\
\hline 72 & $\begin{array}{l}\text { Direct contact for customer } \\
\text { centricity }\end{array}$ & 3 & 8 & 3 & 8 & 3 & 8 & Digit* & $\begin{array}{l}\text { Holotiuk } \\
\text { and Beimborn }\end{array}$ & 2017 & success & explicit \\
\hline 73 & $\begin{array}{l}\text { Customer integration with } \\
\text { open innovation }\end{array}$ & 3 & 8 & 3 & 8 & 3 & 8 & Digit* & $\begin{array}{l}\text { Holotiuk } \\
\text { and Beimborn }\end{array}$ & 2017 & success & explicit \\
\hline 74 & Provide financial resources & 3 & 13 & 3 & 13 & 3 & 13 & Digit* & $\begin{array}{l}\text { Holotiuk } \\
\text { and Beimborn }\end{array}$ & 2017 & success & explicit \\
\hline 75 & $\begin{array}{l}\text { Network effects with open } \\
\text { systems and partner integra- } \\
\text { tion }\end{array}$ & 3 & 12 & 3 & 12 & 3 & 12 & Digit* & $\begin{array}{l}\text { Holotiuk } \\
\text { and Beimborn }\end{array}$ & 2017 & success & explicit \\
\hline 76 & External partners & 3 & 12 & 3 & 12 & 3 & 12 & Digit* & $\begin{array}{l}\text { Holotiuk } \\
\text { and Beimborn }\end{array}$ & 2017 & success & explicit \\
\hline 77 & $\begin{array}{l}\text { Organizational alignment to- } \\
\text { wards digital }\end{array}$ & 5 & 26 & 5 & 26 & 5 & 26 & Digit* & $\begin{array}{l}\text { Holotiuk } \\
\text { and Beimborn }\end{array}$ & 2017 & success & explicit \\
\hline 78 & $\begin{array}{l}\text { Common set of values with } \\
\text { digital as value creation }\end{array}$ & 5 & 26 & 5 & 26 & 5 & 26 & Digit* & $\begin{array}{l}\text { Holotiuk } \\
\text { and Beimborn }\end{array}$ & 2017 & success & explicit \\
\hline 79 & $\begin{array}{l}\text { Not just business but operating } \\
\text { models change }\end{array}$ & 5 & 26 & 5 & 26 & 1 & 1 & Digit* & $\begin{array}{l}\text { Holotiuk } \\
\text { and Beimborn }\end{array}$ & 2017 & success & explicit \\
\hline 80 & $\begin{array}{l}\text { Seamlessly integrated of- } \\
\text { fline(physical) and online } \\
\text { (digital) channels }\end{array}$ & 6 & 27 & 6 & 27 & 6 & 27 & Digit* & $\begin{array}{l}\text { Holotiuk } \\
\text { and Beimborn }\end{array}$ & 2017 & success & explicit \\
\hline 81 & $\begin{array}{l}\text { Multi-level and multi-speed } \\
\text { organization for faster reaction }\end{array}$ & 6 & 28 & 6 & 28 & 6 & 28 & Digit* & $\begin{array}{l}\text { Holotiuk } \\
\text { and Beimborn }\end{array}$ & 2017 & success & explicit \\
\hline 82 & $\begin{array}{l}\text { Organizational separation --> } \\
\text { Spin-off }\end{array}$ & 6 & 28 & 6 & 28 & 6 & 28 & Digit* & $\begin{array}{l}\text { Holotiuk } \\
\text { and Beimborn }\end{array}$ & 2017 & success & explicit \\
\hline 83 & Lean decision-making & 6 & 28 & 6 & 28 & 6 & 28 & Digit* & $\begin{array}{l}\text { Holotiuk } \\
\text { and Beimborn }\end{array}$ & 2017 & success & explicit \\
\hline 84 & $\begin{array}{l}\text { Establish a clear vision with } \\
\text { future positioning }\end{array}$ & 6 & 28 & 6 & 28 & 6 & 28 & Digit* & $\begin{array}{l}\text { Holotiuk } \\
\text { and Beimborn }\end{array}$ & 2017 & success & explicit \\
\hline 85 & $\begin{array}{l}\text { Use data and information from } \\
\text { central source }\end{array}$ & 6 & 27 & 6 & 27 & 6 & 27 & Digit* & $\begin{array}{l}\text { Holotiuk } \\
\text { and Beimborn }\end{array}$ & 2017 & success & explicit \\
\hline 86 & $\begin{array}{l}\text { Fundamentally different role } \\
\text { of IT with two-speed IT }\end{array}$ & 6 & 27 & 6 & 27 & 6 & 27 & Digit* & $\begin{array}{l}\text { Holotiuk } \\
\text { and Beimborn }\end{array}$ & 2017 & success & explicit \\
\hline 87 & $\begin{array}{l}\text { Real-time and large-scale data } \\
\text { processing }\end{array}$ & 6 & 27 & 6 & 27 & 6 & 27 & Digit* & $\begin{array}{l}\text { Holotiuk } \\
\text { and Beimborn }\end{array}$ & 2017 & success & explicit \\
\hline 88 & Modular IT platform & 6 & 27 & 6 & 27 & 6 & 27 & Digit* & $\begin{array}{l}\text { Holotiuk } \\
\text { and Beimborn }\end{array}$ & 2017 & success & explicit \\
\hline 89 & $\begin{array}{l}\text { [Benchmarking }] \text { Is limited in } \\
\text { ambition by the best in prac- } \\
\text { tice which may not be best in a } \\
\text { changing world }\end{array}$ & 1 & 2 & 1 & 2 & 1 & 2 & BPI & $\begin{array}{l}\text { Siha } \\
\text { and Saad }\end{array}$ & 2008 & failure & explicit \\
\hline 90 & $\begin{array}{l}\text { Strategic selection of six } \\
\text { sigma projects and participants }\end{array}$ & 4 & 17 & 4 & 17 & 4 & 17 & BPI & $\begin{array}{l}\text { Siha } \\
\text { and Saad }\end{array}$ & 2008 & success & explicit \\
\hline 91 & $\begin{array}{l}\text { Focus on improve of func- } \\
\text { tional area which does not lead } \\
\text { to profit increase }\end{array}$ & 4 & 23 & 4 & 23 & 2 & 7 & BPI & $\begin{array}{l}\text { Siha } \\
\text { and Saad }\end{array}$ & 2008 & failure & explicit \\
\hline 92 & $\begin{array}{l}\text { Use of 6sigma assumes the } \\
\text { process is sound and just } \\
\text { needs improvement, yet the } \\
\text { process may need redesign, } \\
\text { and creative orientation that }\end{array}$ & 4 & 19 & 4 & 19 & 4 & 19 & BPI & $\begin{array}{l}\text { Siha } \\
\text { and Saad }\end{array}$ & 2008 & failure & explicit \\
\hline
\end{tabular}




\begin{tabular}{|c|c|c|c|c|c|c|c|c|c|c|c|c|}
\hline & $\begin{array}{l}\text { six sigma is not equipped to } \\
\text { fulfil }\end{array}$ & & & & & & & & & & & \\
\hline 93 & $\begin{array}{l}\text { Ease of monitoring one di- } \\
\text { mensional gap analysis }\end{array}$ & 4 & 19 & 4 & 19 & 4 & 19 & BPI & \begin{tabular}{|l|} 
Siha \\
and Saad
\end{tabular} & 2008 & success & explicit \\
\hline 94 & $\begin{array}{l}\text { Mgt. of proprietary info. and } \\
\text { antitrust laws can pose prob- } \\
\text { lems }\end{array}$ & 4 & 17 & 4 & 17 & 4 & 17 & BPI & $\begin{array}{l}\text { Siha } \\
\text { and Saad }\end{array}$ & 2008 & failure & explicit \\
\hline 95 & $\begin{array}{l}\text { Deployment of the most tal- } \\
\text { ented, competent and creative } \\
\text { people in the project }\end{array}$ & 4 & 24 & 4 & 24 & 4 & 24 & BPI & $\begin{array}{l}\text { Siha } \\
\text { and Saad }\end{array}$ & 2008 & success & explicit \\
\hline 96 & $\begin{array}{l}\text { Negligence of the work envi- } \\
\text { ronment aspects of the design } \\
\text { process }\end{array}$ & 4 & 20 & 4 & 20 & 4 & 20 & BPI & $\begin{array}{l}\text { Siha } \\
\text { and Saad }\end{array}$ & 2008 & failure & explicit \\
\hline 97 & $\begin{array}{l}\text { Consideration of human fac- } \\
\text { tors as cost that needs to be re- } \\
\text { duced, rather than a resource } \\
\text { to be developed }\end{array}$ & 4 & 17 & 4 & 17 & 4 & 17 & BPI & $\begin{array}{l}\text { Siha } \\
\text { and Saad }\end{array}$ & 2008 & failure & explicit \\
\hline 98 & $\begin{array}{l}\text { The focus on the measures of } \\
\text { process success }\end{array}$ & 4 & 21 & 4 & 21 & 4 & 21 & BPI & $\begin{array}{l}\text { Siha } \\
\text { and Saad }\end{array}$ & 2008 & success & explicit \\
\hline 99 & $\begin{array}{l}\text { Focus on both the process's } \\
\text { efficiency and effectiveness }\end{array}$ & 4 & 19 & 4 & 19 & 4 & 21 & BPI & \begin{tabular}{|l|} 
Siha \\
and Saad
\end{tabular} & 2008 & success & explicit \\
\hline 100 & $\begin{array}{l}\text { Accuracy in collecting the } \\
\text { process data }\end{array}$ & 4 & 19 & 4 & 19 & 4 & 19 & BPI & $\begin{array}{l}\text { Siha } \\
\text { and Saad }\end{array}$ & 2008 & success & explicit \\
\hline 101 & $\begin{array}{l}\text { Creation of autonomous and } \\
\text { cross-functional teams ac- } \\
\text { countable for the results }\end{array}$ & 4 & 24 & 4 & 24 & 6 & 28 & BPI & $\begin{array}{l}\text { Siha } \\
\text { and Saad }\end{array}$ & 2008 & success & explicit \\
\hline 102 & $\begin{array}{l}\text { The inclusion of the decision } \\
\text { points which are a key to ef- } \\
\text { fective analysis }\end{array}$ & 4 & 19 & 4 & 19 & 4 & 19 & BPI & $\begin{array}{l}\text { Siha } \\
\text { and Saad }\end{array}$ & 2008 & success & explicit \\
\hline 103 & $\begin{array}{l}\text { Failure to define a beginning } \\
\text { and end to the process }\end{array}$ & 4 & 20 & 4 & 20 & 4 & 20 & BPI & \begin{tabular}{|l|} 
Siha \\
and Saad
\end{tabular} & 2008 & failure & explicit \\
\hline 104 & $\begin{array}{l}\text { Inability of defining the pro- } \\
\text { cess boundaries; its beginning } \\
\text { and end }\end{array}$ & 4 & 20 & 4 & 20 & 4 & 20 & BPI & $\begin{array}{l}\text { Siha } \\
\text { and Saad }\end{array}$ & 2008 & failure & explicit \\
\hline 105 & Process improvement & 4 & 19 & 4 & 19 & 4 & 19 & BPI & $\begin{array}{l}\text { Siha } \\
\text { and Saad }\end{array}$ & 2008 & success & implicit \\
\hline 106 & Human resources & 4 & 24 & 4 & 24 & 4 & 24 & BPI & $\begin{array}{l}\text { Siha } \\
\text { and Saad }\end{array}$ & 2008 & success & implicit \\
\hline 107 & Performance measures & 4 & 21 & 4 & 21 & 4 & 22 & BPI & $\begin{array}{l}\text { Siha } \\
\text { and Saad }\end{array}$ & 2008 & success & implicit \\
\hline 108 & $\begin{array}{l}\text { Lack of direct impact on cus- } \\
\text { tomer }\end{array}$ & 2 & 6 & 2 & 6 & 2 & 6 & BPI & \begin{tabular}{|l|} 
Siha \\
and Saad
\end{tabular} & 2008 & failure & explicit \\
\hline 109 & $\begin{array}{l}\text { Questioning the fundamental } \\
\text { assumptions of a process }\end{array}$ & 2 & 7 & 2 & 7 & 2 & 7 & BPI & $\begin{array}{l}\begin{array}{l}\text { Siha } \\
\text { and Saad }\end{array} \\
\end{array}$ & 2008 & success & explicit \\
\hline 110 & $\begin{array}{l}\text { The process chosen for reengi- } \\
\text { neering should be in the center } \\
\text { of the organization for the im- } \\
\text { provement to be felt }\end{array}$ & 2 & 7 & 2 & 7 & 5 & 25 & BPI & $\begin{array}{l}\text { Siha } \\
\text { and Saad }\end{array}$ & 2008 & success & explicit \\
\hline 111 & The focus on the customer & 2 & 6 & 2 & 6 & 2 & 6 & BPI & \begin{tabular}{|l|} 
Siha \\
and Saad
\end{tabular} & 2008 & success & explicit \\
\hline 112 & Management involvement & 3 & 15 & 3 & 15 & 3 & 15 & BPI & $\begin{array}{l}\begin{array}{l}\text { Siha } \\
\text { and Saad }\end{array} \\
\end{array}$ & 2008 & success & explicit \\
\hline 113 & $\begin{array}{l}\text { Training on six sigma method- } \\
\text { ology, tools and project mgt. } \\
\text { skills }\end{array}$ & 3 & 11 & 3 & 11 & 3 & 11 & BPI & $\begin{array}{l}\text { Siha } \\
\text { and Saad }\end{array}$ & 2008 & success & explicit \\
\hline 114 & $\begin{array}{l}\text { Use of appropriate incentive } \\
\text { systems and training }\end{array}$ & 3 & 9 & 3 & 9 & 3 & 9 & BPI & $\begin{array}{l}\text { Siha } \\
\text { and Saad }\end{array}$ & 2008 & success & explicit \\
\hline
\end{tabular}




\begin{tabular}{|c|c|c|c|c|c|c|c|c|c|c|c|c|}
\hline 115 & $\begin{array}{l}\text { Failure to involve both suppli- } \\
\text { ers and customers }\end{array}$ & 3 & 8 & 3 & 8 & 3 & 8 & BPI & $\begin{array}{l}\text { Siha } \\
\text { and Saad }\end{array}$ & 2008 & failure & explicit \\
\hline 116 & $\begin{array}{l}\text { Sharing forums among com- } \\
\text { parative firms proves very ef- } \\
\text { fective for improving practice }\end{array}$ & 3 & 12 & 3 & 12 & 3 & 12 & BPI & $\begin{array}{l}\text { Siha } \\
\text { and Saad }\end{array}$ & 2008 & success & explicit \\
\hline 117 & $\begin{array}{l}\text { Linking best practice to the } \\
\text { delivery of corporate objec- } \\
\text { tives }\end{array}$ & 3 & 11 & 3 & 11 & 3 & 11 & BPI & $\begin{array}{l}\text { Siha } \\
\text { and Saad }\end{array}$ & 2008 & success & explicit \\
\hline 118 & $\begin{array}{l}\text { Emphasis of knowledge shar- } \\
\text { ing and communication }\end{array}$ & 3 & 11 & 3 & 11 & 3 & 11 & BPI & $\begin{array}{l}\text { Siha } \\
\text { and Saad }\end{array}$ & 2008 & success & explicit \\
\hline 119 & Lack of top mgt. support & 3 & 15 & 3 & 15 & 3 & 15 & BPI & $\begin{array}{l}\text { Siha } \\
\text { and Saad }\end{array}$ & 2008 & failure & explicit \\
\hline 120 & $\begin{array}{l}\text { It requires the cooperation } \\
\text { among varies companies }\end{array}$ & 3 & 12 & 3 & 12 & 3 & 12 & BPI & $\begin{array}{l}\text { Siha } \\
\text { and Saad }\end{array}$ & 2008 & failure & explicit \\
\hline 121 & $\begin{array}{l}\text { Total commitment of the lead- } \\
\text { ership }\end{array}$ & 3 & 15 & 3 & 15 & 3 & 15 & BPI & $\begin{array}{l}\text { Siha } \\
\text { and Saad }\end{array}$ & 2008 & success & explicit \\
\hline 122 & $\begin{array}{l}\text { Strong communication among } \\
\text { the participating team }\end{array}$ & 3 & 11 & 3 & 11 & 3 & 11 & BPI & $\begin{array}{l}\text { Siha } \\
\text { and Saad }\end{array}$ & 2008 & success & explicit \\
\hline 123 & $\begin{array}{l}\text { Assuring full participation of } \\
\text { every one in the process }\end{array}$ & 3 & 9 & 3 & 9 & 3 & 9 & BPI & $\begin{array}{l}\text { Siha } \\
\text { and Saad }\end{array}$ & 2008 & success & explicit \\
\hline 124 & Top management & 3 & 15 & 3 & 15 & 3 & 15 & BPI & $\begin{array}{l}\text { Siha } \\
\text { and Saad }\end{array}$ & 2008 & success & implicit \\
\hline 125 & $\begin{array}{l}\text { Linking six sigma to the corp. } \\
\text { strategy, human resources, } \\
\text { customers and suppliers }\end{array}$ & 5 & 26 & 5 & 26 & 5 & 26 & BPI & $\begin{array}{l}\text { Siha } \\
\text { and Saad }\end{array}$ & 2008 & success & explicit \\
\hline 126 & $\begin{array}{l}\text { Set Challenging goals for six } \\
\text { sigma }\end{array}$ & 5 & 25 & 5 & 25 & 5 & 25 & BPI & $\begin{array}{l}\text { Siha } \\
\text { and Saad }\end{array}$ & 2008 & success & explicit \\
\hline 127 & $\begin{array}{l}\text { No linkage with to overall } \\
\text { business goals and objectives }\end{array}$ & 5 & 26 & 5 & 26 & 5 & 26 & BPI & $\begin{array}{l}\text { Siha } \\
\text { and Saad }\end{array}$ & 2008 & failure & explicit \\
\hline 128 & $\begin{array}{l}\text { Viewing it as a tool, not as a } \\
\text { complete PI methodology }\end{array}$ & 5 & 26 & 5 & 26 & 4 & 23 & BPI & $\begin{array}{l}\text { Siha } \\
\text { and Saad }\end{array}$ & 2008 & failure & explicit \\
\hline 129 & $\begin{array}{l}\text { Lack of clear association be- } \\
\text { tween benchmarking and } \\
\text { profit increase }\end{array}$ & 5 & 25 & 5 & 25 & 5 & 25 & BPI & $\begin{array}{l}\text { Siha } \\
\text { and Saad }\end{array}$ & 2008 & failure & explicit \\
\hline 130 & $\begin{array}{l}\text { benchmarking's focus is on the } \\
\text { tactical issues not on the issues } \\
\text { that affect the entire business }\end{array}$ & 5 & 26 & 5 & 26 & 5 & 26 & BPI & $\begin{array}{l}\text { Siha } \\
\text { and Saad }\end{array}$ & 2008 & failure & explicit \\
\hline 131 & $\begin{array}{l}\text { Integration of BPR with the } \\
\text { Corp. Strategy }\end{array}$ & 5 & 26 & 5 & 26 & 5 & 26 & BPI & $\begin{array}{l}\text { Siha } \\
\text { and Saad }\end{array}$ & 2008 & success & explicit \\
\hline 132 & $\begin{array}{l}\text { The ambitious goals of the } \\
\text { reengineering process }\end{array}$ & 5 & 25 & 5 & 25 & 5 & 25 & BPI & $\begin{array}{l}\text { Siha } \\
\text { and Saad }\end{array}$ & 2008 & success & explicit \\
\hline 133 & $\begin{array}{l}\text { The importance of BPR pro- } \\
\text { jects }\end{array}$ & 5 & 25 & 5 & 25 & 5 & 26 & BPI & $\begin{array}{l}\text { Siha } \\
\text { and Saad }\end{array}$ & 2008 & failure & explicit \\
\hline 134 & $\begin{array}{l}\text { Emphasis on achieving the } \\
\text { company's goals }\end{array}$ & 5 & 25 & 5 & 25 & 5 & 25 & BPI & $\begin{array}{l}\text { Siha } \\
\text { and Saad }\end{array}$ & 2008 & success & explicit \\
\hline 135 & $\begin{array}{l}\text { Failure to link the goal of PI to } \\
\text { the organization competitive } \\
\text { priorities }\end{array}$ & 5 & 25 & 5 & 25 & 5 & 25 & BPI & $\begin{array}{l}\text { Siha } \\
\text { and Saad }\end{array}$ & 2008 & failure & explicit \\
\hline 136 & Strategic alignment & 5 & 26 & 5 & 26 & 5 & 26 & BPI & $\begin{array}{l}\text { Siha } \\
\text { and Saad }\end{array}$ & 2008 & success & implicit \\
\hline 137 & $\begin{array}{l}\text { Adjustment of culture and em- } \\
\text { ployees' attitude }\end{array}$ & 6 & 28 & 6 & 28 & 6 & 28 & BPI & $\begin{array}{l}\text { Siha } \\
\text { and Saad }\end{array}$ & 2008 & success & explicit \\
\hline 138 & A supporting infrastructure & 6 & 27 & 6 & 27 & 6 & 27 & BPI & $\begin{array}{l}\text { Siha } \\
\text { and Saad }\end{array}$ & 2008 & success & explicit \\
\hline 139 & $\begin{array}{l}\text { The rigidity of the infrastruc- } \\
\text { ture system }\end{array}$ & 6 & 28 & 6 & 28 & 6 & 28 & BPI & $\begin{array}{l}\text { Siha } \\
\text { and Saad }\end{array}$ & 2008 & failure & explicit \\
\hline
\end{tabular}




\begin{tabular}{|c|c|c|c|c|c|c|c|c|c|c|c|c|}
\hline 140 & Business environment & 6 & 28 & 6 & 28 & 6 & 28 & BPI & $\begin{array}{l}\text { Siha } \\
\text { and Saad }\end{array}$ & 2008 & success & implicit \\
\hline 141 & $\begin{array}{l}\text { The effective use of infor- } \\
\text { mation and communication } \\
\text { technology }\end{array}$ & 7 & 33 & 7 & 33 & 6 & 27 & BPI & $\begin{array}{l}\text { Siha } \\
\text { and Saad }\end{array}$ & 2008 & success & explicit \\
\hline 142 & $\begin{array}{l}\text { Company-wide education on } \\
\text { risk management }\end{array}$ & 1 & 5 & 1 & 5 & 1 & 5 & PPM & Costantino et al. & 2015 & success & implicit \\
\hline 143 & $\begin{array}{l}\text { Organization's processes for } \\
\text { assigning ownership of risks }\end{array}$ & 1 & 5 & 1 & 5 & 1 & 5 & PPM & Costantino et al. & 2015 & success & implicit \\
\hline 144 & Risk register & 1 & 5 & 1 & 5 & 1 & 5 & PPM & Costantino et al. & 2015 & success & implicit \\
\hline 145 & Project schedule/plan & 4 & 23 & 4 & 23 & 4 & 23 & PPM & Costantino et al. & 2015 & success & explicit \\
\hline 146 & Personnel & 4 & 24 & 4 & 24 & 4 & 24 & PPM & Costantino et al. & 2015 & success & explicit \\
\hline 147 & Monitoring and feedback & 4 & 22 & 4 & 22 & 3 & 11 & PPM & Costantino et al. & 2015 & success & explicit \\
\hline 148 & Troubleshooting & 4 & 17 & 1 & 5 & 1 & 5 & PPM & Costantino et al. & 2015 & success & explicit \\
\hline 149 & Human resource management & 4 & 24 & 4 & 24 & 4 & 24 & PPM & Costantino et al. & 2015 & success & implicit \\
\hline 150 & $\begin{array}{l}\text { Up-to-date risk management } \\
\text { plan }\end{array}$ & 4 & 23 & 4 & 23 & 4 & 23 & PPM & Costantino et al. & 2015 & success & implicit \\
\hline 151 & Client acceptance & 2 & 6 & 2 & 6 & 2 & 6 & PPM & Costantino et al. & 2015 & success & xplicit \\
\hline 152 & Top management support & 3 & 15 & 3 & 15 & 3 & 15 & PPM & Costantino et al. & 2015 & success & explicit \\
\hline 153 & Client consultation & 3 & 8 & 3 & 8 & 3 & 8 & PPM & Costantino et al. & 2015 & success & explicit \\
\hline 154 & Communication & 3 & 10 & 3 & 10 & 3 & 10 & PPM & Costantino et al. & 2015 & success & explicit \\
\hline 155 & Project sponsorship & 3 & 13 & 3 & 13 & 3 & 13 & PPM & Costantino et al. & 2015 & success & implicit \\
\hline 156 & Project mission & 6 & 28 & 6 & 28 & 6 & 28 & PPM & Costantino et al. & 2015 & success & explicit \\
\hline 157 & Technical tasks & 7 & 29 & 7 & 29 & 7 & 29 & PPM & Costantino et al. & 2015 & success & xplicit \\
\hline 158 & Risk management & 1 & 5 & 1 & 5 & 1 & 5 & PM & $\begin{array}{l}\text { Pankratz } \\
\text { and Loebbecke }\end{array}$ & 2011 & success & implicit \\
\hline 159 & Agile development & 1 & 1 & 1 & 1 & 6 & 28 & PM & $\begin{array}{l}\text { Pankratz } \\
\text { and Loebbecke }\end{array}$ & 2011 & success & implicit \\
\hline 160 & Safety in Project & 1 & 5 & 1 & 5 & 5 & 25 & PM & $\begin{array}{l}\text { Pankratz } \\
\text { and Loebbecke }\end{array}$ & 2011 & success & explicit \\
\hline 161 & Size and value of a project & 4 & 23 & 4 & 23 & 4 & 23 & PM & $\begin{array}{l}\text { Pankratz } \\
\text { and Loebbecke }\end{array}$ & 2011 & success & implicit \\
\hline 162 & $\begin{array}{l}\text { Uniqueness of project activi- } \\
\text { ties }\end{array}$ & 4 & 23 & 4 & 23 & 4 & 23 & PM & $\begin{array}{l}\text { Pankratz } \\
\text { and Loebbecke }\end{array}$ & 2011 & success & implicit \\
\hline 163 & Team member's skills & 4 & 16 & 4 & 16 & 4 & 24 & PM & $\begin{array}{l}\text { Pankratz } \\
\text { and Loebbecke }\end{array}$ & 2011 & success & implicit \\
\hline 164 & Project manager's skills & 4 & 17 & 4 & 17 & 4 & 17 & PM & $\begin{array}{l}\text { Pankratz } \\
\text { and Loebbecke }\end{array}$ & 2011 & success & implicit \\
\hline 165 & $\begin{array}{l}\text { Project manager skills and } \\
\text { competencies }\end{array}$ & 4 & 17 & 4 & 17 & 4 & 17 & PM & $\begin{array}{l}\text { Pankratz } \\
\text { and Loebbecke }\end{array}$ & 2011 & success & explicit \\
\hline 166 & $\begin{array}{l}\text { Performance measurement } \\
\text { systems }\end{array}$ & 4 & 22 & 4 & 22 & 4 & 22 & PM & $\begin{array}{l}\text { Pankratz } \\
\text { and Loebbecke }\end{array}$ & 2011 & success & explicit \\
\hline 167 & Start on the right foot & 4 & 23 & 4 & 23 & 4 & 23 & PM & $\begin{array}{l}\text { Pankratz } \\
\text { and Loebbecke }\end{array}$ & 2011 & success & explicit \\
\hline 168 & Maintain momentum & 4 & 17 & 4 & 17 & 1 & 3 & $\mathrm{PM}$ & $\begin{array}{l}\text { Pankratz } \\
\text { and Loebbecke }\end{array}$ & 2011 & success & explicit \\
\hline 169 & Track progress & 4 & 21 & 4 & 21 & 4 & 22 & $\mathrm{PM}$ & $\begin{array}{l}\text { Pankratz } \\
\text { and Loebbecke }\end{array}$ & 2011 & success & explicit \\
\hline 170 & Make smart decisions & 4 & 17 & 4 & 17 & 4 & 17 & $\mathrm{PM}$ & $\begin{array}{l}\text { Pankratz } \\
\text { and Loebbecke }\end{array}$ & 2011 & success & explicit \\
\hline 171 & $\begin{array}{l}\text { Insitutionalize post-mortem } \\
\text { analyses }\end{array}$ & 4 & 19 & 4 & 19 & 3 & 11 & $\mathrm{PM}$ & $\begin{array}{l}\text { Pankratz } \\
\text { and Loebbecke }\end{array}$ & 2011 & success & explicit \\
\hline 172 & Management practices & 4 & 17 & 4 & 17 & 4 & 17 & PM & $\begin{array}{l}\text { Pankratz } \\
\text { and Loebbecke }\end{array}$ & 2011 & success & implicit \\
\hline
\end{tabular}




\begin{tabular}{|c|c|c|c|c|c|c|c|c|c|c|c|c|}
\hline 173 & Metrics & 4 & 21 & 4 & 21 & 4 & 22 & PM & $\begin{array}{l}\text { Pankratz } \\
\text { and Loebbecke }\end{array}$ & 2011 & success & implicit \\
\hline 174 & $\begin{array}{l}\text { Control of the development } \\
\text { process }\end{array}$ & 4 & 21 & 4 & 21 & 4 & 21 & PM & $\begin{array}{l}\text { Pankratz } \\
\text { and Loebbecke }\end{array}$ & 2011 & success & mplicit \\
\hline 175 & Staged delivery & 4 & 22 & 4 & 22 & 4 & 23 & PM & $\begin{array}{l}\text { Pankratz } \\
\text { and Loebbecke }\end{array}$ & 2011 & success & implicit \\
\hline 176 & Planning maturity & 4 & 23 & 4 & 23 & 4 & 23 & PM & $\begin{array}{l}\text { Pankratz } \\
\text { and Loebbecke }\end{array}$ & 2011 & success & implicit \\
\hline 177 & $\begin{array}{l}\text { Formal definition and meas- } \\
\text { urement of IS project success }\end{array}$ & 4 & 22 & 4 & 22 & 4 & 22 & PM & $\begin{array}{l}\text { Pankratz } \\
\text { and Loebbecke }\end{array}$ & 2011 & success & implicit \\
\hline 178 & $\begin{array}{l}\text { Control mechanisms in inter- } \\
\text { nal and outsourced IS projects }\end{array}$ & 4 & 22 & 4 & 22 & 4 & 22 & PM & $\begin{array}{l}\text { Pankratz } \\
\text { and Loebbecke }\end{array}$ & 2011 & success & implicit \\
\hline 179 & $\begin{array}{l}\text { Efficiency of Project Perfor- } \\
\text { mance }\end{array}$ & 4 & 22 & 4 & 22 & 4 & 22 & PM & $\begin{array}{l}\text { Pankratz } \\
\text { and Loebbecke }\end{array}$ & 2011 & success & explicit \\
\hline 180 & Team Members' Qualification & 4 & 24 & 4 & 24 & 4 & 24 & PM & $\begin{array}{l}\text { Pankratz } \\
\text { and Loebbecke }\end{array}$ & 2011 & success & explicit \\
\hline 181 & Right Mix of Team Members & 4 & 24 & 4 & 24 & 4 & 24 & PM & $\begin{array}{l}\text { Pankratz } \\
\text { and Loebbecke }\end{array}$ & 2011 & success & explicit \\
\hline 182 & $\begin{array}{l}\text { Team Members' Responsibil- } \\
\text { ity }\end{array}$ & 4 & 24 & 4 & 24 & 4 & 24 & PM & $\begin{array}{l}\text { Pankratz } \\
\text { and Loebbecke }\end{array}$ & 2011 & success & xplicit \\
\hline 183 & Monitoring, Controls & 4 & 21 & 4 & 21 & 4 & 22 & PM & $\begin{array}{l}\text { Pankratz } \\
\text { and Loebbecke }\end{array}$ & 2011 & success & explicit \\
\hline 184 & Planning & 4 & 23 & 4 & 23 & 4 & 23 & PM & $\begin{array}{l}\text { Pankratz } \\
\text { and Loebbecke }\end{array}$ & 2011 & success & xplicit \\
\hline 185 & Systematic Approach & 4 & 23 & 4 & 23 & 4 & 23 & PM & $\begin{array}{l}\text { Pankratz } \\
\text { and Loebbecke }\end{array}$ & 2011 & success & xplicit \\
\hline 186 & $\begin{array}{l}\text { Project Manager's Characteris- } \\
\text { tics }\end{array}$ & 4 & 17 & 4 & 24 & 4 & 17 & PM & $\begin{array}{l}\text { Pankratz } \\
\text { and Loebbecke }\end{array}$ & 2011 & success & xplicit \\
\hline 187 & Project characteristics & 2 & 7 & 2 & 7 & 4 & 23 & PM & $\begin{array}{l}\text { Pankratz } \\
\text { and Loebbecke }\end{array}$ & 2011 & success & implicit \\
\hline 188 & Work processes & 2 & 7 & 2 & 7 & 2 & 7 & PM & $\begin{array}{l}\text { Pankratz } \\
\text { and Loebbecke }\end{array}$ & 2011 & success & implicit \\
\hline 189 & Standards and procedures & 2 & 7 & 2 & 7 & 2 & 7 & PM & $\begin{array}{l}\text { Pankratz } \\
\text { and Loebbecke }\end{array}$ & 2011 & success & implicit \\
\hline 190 & Assuring Product Quality & 2 & 6 & 4 & 22 & 4 & 22 & PM & $\begin{array}{l}\text { Pankratz } \\
\text { and Loebbecke }\end{array}$ & 2011 & success & explicit \\
\hline 191 & Top management support & 3 & 15 & 3 & 15 & 3 & 15 & PM & $\begin{array}{l}\text { Pankratz } \\
\text { and Loebbecke }\end{array}$ & 2011 & success & implicit \\
\hline 192 & $\begin{array}{l}\text { Supporting management prac- } \\
\text { tices }\end{array}$ & 3 & 15 & 3 & 15 & 3 & 15 & $\mathrm{PM}$ & $\begin{array}{l}\text { Pankratz } \\
\text { and Loebbecke }\end{array}$ & 2011 & success & explicit \\
\hline 193 & Team dynamics & 3 & 14 & 3 & 14 & 3 & 14 & PM & $\begin{array}{l}\text { Pankratz } \\
\text { and Loebbecke }\end{array}$ & 2011 & success & implicit \\
\hline 194 & $\begin{array}{l}\text { Senior management support } \\
\text { for strategic projects }\end{array}$ & 3 & 15 & 3 & 15 & 3 & 15 & $\mathrm{PM}$ & $\begin{array}{l}\text { Pankratz } \\
\text { and Loebbecke }\end{array}$ & 2011 & success & implicit \\
\hline 195 & User participation & 3 & 8 & 3 & 8 & 3 & 8 & PM & $\begin{array}{l}\text { Pankratz } \\
\text { and Loebbecke }\end{array}$ & 2011 & success & implicit \\
\hline 196 & Joint application development & 3 & 12 & 3 & 12 & 3 & 8 & PM & $\begin{array}{l}\text { Pankratz } \\
\text { and Loebbecke }\end{array}$ & 2011 & success & implicit \\
\hline 197 & User partnering & 3 & 8 & 3 & 8 & 3 & 11 & PM & $\begin{array}{l}\text { Pankratz } \\
\text { and Loebbecke }\end{array}$ & 2011 & success & implicit \\
\hline 198 & (End-user) training & 3 & 8 & 3 & 8 & 3 & 11 & PM & $\begin{array}{l}\text { Pankratz } \\
\text { and Loebbecke }\end{array}$ & 2011 & success & implicit \\
\hline 199 & Top management support & 3 & 15 & 3 & 15 & 3 & 15 & PM & $\begin{array}{l}\text { Pankratz } \\
\text { and Loebbecke }\end{array}$ & 2011 & success & implicit \\
\hline 200 & $\begin{array}{l}\text { Relationship Client - Contrac- } \\
\text { tor }\end{array}$ & 3 & 8 & 3 & 8 & 2 & 6 & PM & $\begin{array}{l}\text { Pankratz } \\
\text { and Loebbecke }\end{array}$ & 2011 & success & explicit \\
\hline
\end{tabular}




\begin{tabular}{|c|c|c|c|c|c|c|c|c|c|c|c|c|}
\hline 201 & $\begin{array}{l}\text { Relationship Management- } \\
\text { Project }\end{array}$ & 3 & 15 & 3 & 15 & 3 & 15 & PM & $\begin{array}{l}\text { Pankratz } \\
\text { and Loebbecke }\end{array}$ & 2011 & success & explicit \\
\hline 202 & Team Members' Motivation & 3 & 14 & 3 & 14 & 3 & 14 & PM & $\begin{array}{l}\text { Pankratz } \\
\text { and Loebbecke }\end{array}$ & 2011 & success & explicit \\
\hline 203 & $\begin{array}{l}\text { Team Members' Focus on Pro- } \\
\text { ject }\end{array}$ & 3 & 14 & 3 & 14 & 3 & 9 & PM & $\begin{array}{l}\text { Pankratz } \\
\text { and Loebbecke }\end{array}$ & 2011 & success & explicit \\
\hline 204 & Communication in Project & 3 & 11 & 3 & 11 & 3 & 11 & PM & $\begin{array}{l}\text { Pankratz } \\
\text { and Loebbecke }\end{array}$ & 2011 & success & explicit \\
\hline 205 & Urgency of a project outcome & 5 & 25 & 5 & 25 & 5 & 25 & PM & $\begin{array}{l}\text { Pankratz } \\
\text { and Loebbecke }\end{array}$ & 2011 & success & implicit \\
\hline 206 & Political factors & 5 & 26 & 5 & 26 & 5 & 26 & PM & $\begin{array}{l}\text { Pankratz } \\
\text { and Loebbecke }\end{array}$ & 2011 & success & implicit \\
\hline 207 & Economic factors & 5 & 26 & 5 & 26 & 5 & 26 & PM & $\begin{array}{l}\text { Pankratz } \\
\text { and Loebbecke }\end{array}$ & 2011 & success & implicit \\
\hline 208 & Different project goals & 5 & 25 & 5 & 25 & 5 & 25 & PM & $\begin{array}{l}\text { Pankratz } \\
\text { and Loebbecke }\end{array}$ & 2011 & success & implicit \\
\hline 209 & $\begin{array}{l}\text { Management of user expecta- } \\
\text { tions }\end{array}$ & 5 & 25 & 5 & 25 & 3 & 8 & $\mathrm{PM}$ & $\begin{array}{l}\text { Pankratz } \\
\text { and Loebbecke }\end{array}$ & 2011 & success & implicit \\
\hline 210 & $\begin{array}{l}\text { Management of Team Mem- } \\
\text { bers' Expectations }\end{array}$ & 5 & 25 & 5 & 25 & 4 & 17 & $\mathrm{PM}$ & $\begin{array}{l}\text { Pankratz } \\
\text { and Loebbecke }\end{array}$ & 2011 & success & explicit \\
\hline 211 & Clear Objective & 5 & 25 & 5 & 25 & 5 & 25 & PM & $\begin{array}{l}\text { Pankratz } \\
\text { and Loebbecke }\end{array}$ & 2011 & success & explicit \\
\hline 212 & Transparency in Project & 5 & 25 & 5 & 25 & 5 & 25 & PM & $\begin{array}{l}\text { Pankratz } \\
\text { and Loebbecke }\end{array}$ & 2011 & success & explicit \\
\hline 213 & Social factors & 6 & 28 & 6 & 28 & 6 & 28 & PM & $\begin{array}{l}\text { Pankratz } \\
\text { and Loebbecke }\end{array}$ & 2011 & success & implicit \\
\hline 214 & $\begin{array}{l}\text { Organizational } \\
\text { the project leve }\end{array}$ & 6 & 28 & 6 & 28 & 6 & 28 & PM & $\begin{array}{l}\text { Pankratz } \\
\text { and Loebbecke }\end{array}$ & 2011 & success & explicit \\
\hline 215 & Organizational characteristics & 6 & 28 & 6 & 28 & 6 & 28 & $\mathrm{PM}$ & $\begin{array}{l}\text { Pankratz } \\
\text { and Loebbecke }\end{array}$ & 2011 & success & implicit \\
\hline 216 & Organizational practices & 6 & 28 & 6 & 28 & 6 & 28 & PM & $\begin{array}{l}\text { Pankratz } \\
\text { and Loebbecke }\end{array}$ & 2011 & success & implicit \\
\hline 217 & Social integration & 6 & 28 & 6 & 28 & 6 & 28 & PM & $\begin{array}{l}\text { Pankratz } \\
\text { and Loebbecke }\end{array}$ & 2011 & success & implicit \\
\hline 218 & Technology characteristics & 7 & 32 & 7 & 32 & 7 & 33 & PM & $\begin{array}{l}\text { Pankratz } \\
\text { and Loebbecke }\end{array}$ & 2011 & success & implicit \\
\hline 219 & Tools and technology & 7 & 32 & 7 & 33 & 7 & 32 & $\mathrm{PM}$ & $\begin{array}{l}\text { Pankratz } \\
\text { and Loebbecke }\end{array}$ & 2011 & success & implicit \\
\hline 220 & Missing role clarity & T & 24 & . & 24 & 6 & 28 & PPM & Beringer et al. & 2013 & failure & implicit \\
\hline 221 & Role Clarity & 4 & 24 & 4 & 24 & 6 & 28 & PPM & Beringer et al. & 2013 & success & implicit \\
\hline 222 & $\begin{array}{l}\text { Increased engagement in the } \\
\text { PPM process }\end{array}$ & 3 & 15 & 3 & 15 & 3 & 9 & PPM & Beringer et al. & 2013 & success & implicit \\
\hline 223 & $\begin{array}{l}\text { Engagement on project suc- } \\
\text { cess }\end{array}$ & 3 & 14 & 3 & 14 & 3 & 15 & PPM & Beringer et al. & 2013 & success & implicit \\
\hline 224 & $\begin{array}{l}\text { Engagement on success of } \\
\text { projects that are personally } \\
\text { important to the senior man- } \\
\text { ager }\end{array}$ & 3 & 15 & 3 & 15 & 3 & 15 & PPM & Beringer et al. & 2013 & failure & implicit \\
\hline 225 & $\begin{array}{l}\text { Engagement in portfolio struc- } \\
\text { turing in terms of missing } \\
\text { knowledge of the PPManager }\end{array}$ & 3 & 15 & 3 & 15 & 3 & 15 & PPM & Beringer et al. & 2013 & failure & implicit \\
\hline 226 & $\begin{array}{l}\text { Positive influence on strategic } \\
\text { fit }\end{array}$ & 5 & 26 & 5 & 26 & 5 & 26 & PPM & Beringer et al. & 2013 & success & implicit \\
\hline 227 & $\begin{array}{l}\text { aware of changes in schedul- } \\
\text { ing, new requirements or } \\
\text { guideline updates }\end{array}$ & 1 & 3 & 1 & 3 & 1 & 3 & DT & Abollado et al. & 2017 & success & implicit \\
\hline 228 & Workflow Automation & 1 & 1 & 1 & 1 & 4 & 19 & DT & Abollado et al. & 2017 & success & explicit \\
\hline
\end{tabular}




\begin{tabular}{|c|c|c|c|c|c|c|c|c|c|c|c|c|}
\hline 229 & Workflow status tracking tool & 4 & 21 & 4 & 21 & 3 & 11 & DT & Abollado et al. & 2017 & success & explicit \\
\hline 230 & Error-proofed, pre-filled forms & 4 & 23 & 4 & 23 & 3 & 11 & DT & Abollado et al. & 2017 & success & explicit \\
\hline 231 & $\begin{array}{l}\text { Workflow progress tracking } \\
\text { tool }\end{array}$ & 4 & 21 & 4 & 21 & 4 & 21 & DT & Abollado et al. & 2017 & success & explicit \\
\hline 232 & $\begin{array}{l}\text { Workflow Management Soft- } \\
\text { ware }\end{array}$ & 4 & 21 & 4 & 21 & 4 & 21 & DT & Abollado et al. & 2017 & success & explicit \\
\hline 233 & Implement in phases & 4 & 23 & 4 & 23 & 4 & 23 & DT & Abollado et al. & 2017 & success & explicit \\
\hline 234 & Use metrics & 4 & 21 & 4 & 21 & 4 & 21 & DT & Abollado et al. & 2017 & success & explicit \\
\hline 235 & $\begin{array}{l}\text { Analysis of the underlying } \\
\text { process and improve it where } \\
\text { necessary, prior to digitising } \\
\text { the process }\end{array}$ & 2 & 7 & 4 & 19 & 4 & 19 & DT & Abollado et al. & 2017 & success & implicit \\
\hline 236 & $\begin{array}{l}\text { choosing the right processes to } \\
\text { be digitised }\end{array}$ & 2 & 7 & 2 & 7 & 2 & 7 & DT & Abollado et al. & 2017 & failure & implicit \\
\hline 237 & $\begin{array}{l}\text { Focus first on processes that } \\
\text { are fully understood }\end{array}$ & 2 & 7 & 2 & 7 & 2 & 7 & DT & Abollado et al. & 2017 & success & explicit \\
\hline 238 & \begin{tabular}{|l|} 
Embedding bad practices on \\
the system while digitising the \\
process
\end{tabular} & 3 & 11 & 3 & 11 & 3 & 11 & DT & Abollado et al. & 2017 & failure & implicit \\
\hline 239 & Automatic Notifications & 3 & 11 & 3 & 11 & 6 & 27 & DT & Abollado et al. & 2017 & success & explicit \\
\hline 240 & $\begin{array}{l}\text { to map the process heavily de- } \\
\text { pends on employees' commit- } \\
\text { ment }\end{array}$ & 3 & 9 & 3 & 9 & 3 & 9 & DT & Abollado et al. & 2017 & failure & implicit \\
\hline 241 & $\begin{array}{l}\text { Involving the right people } \\
\text { from the beginning }\end{array}$ & 3 & 12 & 3 & 12 & 3 & 12 & DT & Abollado et al. & 2017 & success & implicit \\
\hline 242 & $\begin{array}{l}\text { managing the business and } \\
\text { technical aspects of the system } \\
\text { will create additional work }\end{array}$ & 3 & 15 & 3 & 15 & 3 & 15 & DT & Abollado et al. & 2017 & failure & implicit \\
\hline 243 & support of senior management & 3 & 15 & 3 & 15 & 3 & 15 & DT & Abollado et al. & 2017 & success & explicit \\
\hline 244 & Get the support of end users & 3 & 8 & 3 & 8 & 3 & 8 & DT & Abollado et al. & 2017 & success & explicit \\
\hline 245 & Workflow Automation & 6 & 27 & 6 & 27 & 4 & 19 & DT & Abollado et al. & 2017 & success & explicit \\
\hline 246 & Role assignment tool & 6 & 27 & 6 & 27 & 6 & 27 & DT & Abollado et al. & 2017 & success & explicit \\
\hline 247 & \begin{tabular}{|l|} 
allow data to be pulled from \\
an upstream process or task, to \\
use it as input for another ac- \\
tivity located downstream \\
\end{tabular} & 6 & 27 & 6 & 27 & 4 & 20 & DT & Abollado et al. & 2017 & success & implicit \\
\hline 248 & Tool and databases integration & 6 & 27 & 5 & 27 & 6 & 27 & DT & Abollado et al. & 2017 & success & explicit \\
\hline 249 & $\begin{array}{l}\text { Integrate the digital workflow } \\
\text { with current systems }\end{array}$ & 6 & 27 & 6 & 27 & 6 & 27 & DT & Abollado et al. & 2017 & success & explicit \\
\hline 250 & $\begin{array}{l}\text { workflow management as a } \\
\text { way of reducing their deci- } \\
\text { sion-making power }\end{array}$ & 7 & 33 & 7 & 33 & 7 & 33 & DT & Abollado et al. & 2017 & failure & implicit \\
\hline 251 & Urgency & 4 & 5 & 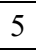 & 25 & 4 & 23 & PPM & $1 \mathrm{Ka}$ & 009 & success & xplicit \\
\hline 252 & $\begin{array}{l}\text { characteristics of the project } \\
\text { team leader }\end{array}$ & 4 & 17 & 4 & 17 & 4 & 17 & PPM & Ika & 2009 & success & explicit \\
\hline 253 & $\begin{array}{l}\text { selecting a development ap- } \\
\text { proach that fits the characteris- } \\
\text { tics of the project }\end{array}$ & 4 & 23 & 4 & 23 & 4 & 23 & $\mathrm{PM}$ & $\begin{array}{l}\text { Kirsch } \\
\text { and Slaughter }\end{array}$ & 2013 & success & explicit \\
\hline 254 & $\begin{array}{l}\text { understanding success factors } \\
\text { of projects that are large, com- } \\
\text { plex, and uncertain }\end{array}$ & 4 & 17 & 4 & 17 & 4 & 17 & PM & $\begin{array}{l}\text { Kirsch } \\
\text { and Slaughter }\end{array}$ & 2013 & failure & explicit \\
\hline 255 & $\begin{array}{l}\text { poorly understood or ill-struc- } \\
\text { tured project requirements }\end{array}$ & 4 & 23 & 4 & 23 & 4 & 23 & $\mathrm{PM}$ & $\begin{array}{l}\text { Kirsch } \\
\text { and Slaughter }\end{array}$ & 2013 & failure & explicit \\
\hline 256 & power differences & 4 & 24 & 4 & 24 & 6 & 28 & $\mathrm{PM}$ & $\begin{array}{l}\text { Kirsch } \\
\text { and Slaughter }\end{array}$ & 2013 & failure & explicit \\
\hline
\end{tabular}




\begin{tabular}{|c|c|c|c|c|c|c|c|c|c|c|c|c|}
\hline 257 & Experience & 4 & 24 & 4 & 24 & 4 & 24 & $\mathrm{PM}$ & $\begin{array}{l}\text { Kirsch } \\
\text { and Slaughter }\end{array}$ & 2013 & success & implicit \\
\hline 258 & Formal and informal control & 4 & 21 & 4 & 21 & 4 & 21 & PM & $\begin{array}{l}\text { Kirsch } \\
\text { and Slaughter }\end{array}$ & 2013 & success & implicit \\
\hline 259 & Coordination mechanisms & 4 & 21 & 4 & 21 & 4 & 21 & $\mathrm{PM}$ & $\begin{array}{l}\text { Kirsch } \\
\text { and Slaughter }\end{array}$ & 2013 & success & implicit \\
\hline 260 & Methodologies & 4 & 17 & 4 & 17 & 4 & 17 & $\mathrm{PM}$ & $\begin{array}{l}\text { Kirsch } \\
\text { and Slaughter }\end{array}$ & 2013 & success & implicit \\
\hline 261 & $\begin{array}{l}\text { the number and diversity of } \\
\text { stakeholders }\end{array}$ & 2 & 6 & 2 & 6 & 2 & 6 & $\mathrm{PM}$ & $\begin{array}{l}\text { Kirsch } \\
\text { and Slaughter }\end{array}$ & 2013 & failure & explicit \\
\hline 262 & $\begin{array}{l}\text { ineffective communication be- } \\
\text { haviors }\end{array}$ & 3 & 11 & 3 & 11 & 3 & 11 & PM & $\begin{array}{l}\text { Kirsch } \\
\text { and Slaughter }\end{array}$ & 2013 & failure & explicit \\
\hline 263 & $\begin{array}{l}\text { differences in priorities, goals, } \\
\text { and agendas of project stake- } \\
\text { holders }\end{array}$ & 5 & 25 & 5 & 25 & 5 & 25 & $\mathrm{PM}$ & $\begin{array}{l}\text { Kirsch } \\
\text { and Slaughter }\end{array}$ & 2013 & failure & explicit \\
\hline 264 & Uncertain project environment & 6 & 28 & 6 & 28 & 6 & 28 & PM & $\begin{array}{l}\text { Kirsch } \\
\text { and Slaughter }\end{array}$ & 2013 & success & implicit \\
\hline 265 & Complex project environment & 6 & 28 & 6 & 28 & 6 & 28 & PM & $\begin{array}{l}\text { Kirsch } \\
\text { and Slaughter }\end{array}$ & 2013 & success & implicit \\
\hline 266 & $\begin{array}{l}\text { Computer-aided software en- } \\
\text { gineering tools }\end{array}$ & 6 & 27 & 6 & 27 & 6 & 27 & PM & \begin{tabular}{|l|} 
Kirsch \\
and Slaughter
\end{tabular} & 2013 & success & implicit \\
\hline 267 & Maturity of Software & 7 & 30 & 7 & 30 & 7 & 30 & PM & $\begin{array}{l}\text { Kirsch } \\
\text { and Slaughter }\end{array}$ & 2013 & success & implicit \\
\hline 268 & Time Compliance & 4 & 23 & 4 & 23 & 4 & 22 & $\mathrm{PM}$ & $\begin{array}{l}\text { Varajão } \\
\text { and Trigo }\end{array}$ & 2016 & $\begin{array}{c}\text { success } \\
\text { measures }\end{array}$ & explicit \\
\hline 269 & Scope Compliance & 4 & 23 & 5 & 25 & 4 & 22 & PM & $\begin{array}{l}\text { Varajão } \\
\text { and Trigo }\end{array}$ & 2016 & $\begin{array}{c}\text { success } \\
\text { measures }\end{array}$ & explicit \\
\hline 270 & Key Performance Indicators & 4 & 22 & 4 & 22 & 4 & 22 & $\mathrm{PM}$ & $\begin{array}{l}\text { Varajão } \\
\text { and Trigo }\end{array}$ & 2016 & $\begin{array}{c}\text { success } \\
\text { measures }\end{array}$ & explicit \\
\hline 271 & $\begin{array}{l}\text { details on the requirements } \\
\text { that are not well perceived at } \\
\text { the initial stages of project }\end{array}$ & 4 & 23 & 4 & 22 & 5 & 25 & $\mathrm{PM}$ & $\begin{array}{l}\text { Varajão } \\
\text { and Trigo }\end{array}$ & 2016 & failure & implicit \\
\hline 272 & $\begin{array}{l}\text { compliance with the client's } \\
\text { business objectives }\end{array}$ & 2 & 6 & 2 & 6 & 2 & 6 & $\mathrm{PM}$ & & 2016 & $\begin{array}{c}\text { success } \\
\text { measures }\end{array}$ & explicit \\
\hline 273 & user satisfaction & 2 & 6 & 2 & 6 & 2 & 6 & $\mathrm{PM}$ & $\begin{array}{l}\text { Varajão } \\
\text { and Trigo }\end{array}$ & 2016 & $\begin{array}{c}\text { success } \\
\text { measures }\end{array}$ & explicit \\
\hline 274 & customer satisfaction & 2 & 6 & 2 & 6 & 2 & 6 & PM & $\begin{array}{l}\text { Varajão } \\
\text { and Trigo }\end{array}$ & 2016 & $\begin{array}{c}\text { success } \\
\text { measures }\end{array}$ & explicit \\
\hline 275 & $\begin{array}{l}\text { quality of resulting prod- } \\
\text { ucts/services (deliverables) }\end{array}$ & 2 & 6 & 2 & 6 & 2 & 6 & PM & $\begin{array}{l}\text { Varajão } \\
\text { and Trigo }\end{array}$ & 2016 & $\begin{array}{c}\text { success } \\
\text { measures }\end{array}$ & explicit \\
\hline 276 & Budget Compliance & 3 & 13 & 3 & 13 & 3 & 13 & PM & $\begin{array}{l}\text { Varajão } \\
\text { and Trigo }\end{array}$ & 2016 & $\begin{array}{c}\text { success } \\
\text { measures }\end{array}$ & explicit \\
\hline 277 & operational team satisfaction & 3 & 14 & 3 & 14 & 3 & 14 & PM & \begin{tabular}{|l|} 
Varajão \\
and Trigo \\
\end{tabular} & 2016 & $\begin{array}{c}\text { success } \\
\text { measures }\end{array}$ & explicit \\
\hline 278 & sponsor satisfaction & 3 & 8 & 3 & 8 & 2 & 6 & PM & $\begin{array}{l}\text { Varajão } \\
\text { and Trigo } \\
\end{array}$ & 2016 & $\begin{array}{c}\text { success } \\
\text { measures }\end{array}$ & explicit \\
\hline 279 & $\begin{array}{l}\begin{array}{l}\text { use of IS solutions by the cus- } \\
\text { tomer }\end{array} \\
\end{array}$ & 3 & 8 & 7 & 31 & 3 & 7 & $\mathrm{PM}$ & $\begin{array}{l}\text { Varajão } \\
\text { and Trigo }\end{array}$ & 2016 & $\begin{array}{c}\text { success } \\
\text { measures }\end{array}$ & explicit \\
\hline 280 & $\begin{array}{l}\text { changes in the scope requested } \\
\text { by the customer }\end{array}$ & 3 & 8 & 3 & 8 & 3 & 8 & $\mathrm{PM}$ & $\begin{array}{l}\text { Varajão } \\
\text { and Trigo }\end{array}$ & 2016 & failure & implicit \\
\hline 281 & $\begin{array}{l}\text { compliance with the business } \\
\text { goals set for the project }\end{array}$ & 5 & 26 & 5 & 26 & 5 & 26 & PM & $\begin{array}{l}\text { Varajão } \\
\text { and Trigo }\end{array}$ & 2016 & $\begin{array}{c}\text { success } \\
\text { measures }\end{array}$ & explicit \\
\hline 282 & $\begin{array}{l}\text { contribution to the develop- } \\
\text { ment of the organization }\end{array}$ & 5 & 26 & 5 & 26 & 3 & 11 & PM & $\begin{array}{l}\text { Varajão } \\
\text { and Trigo }\end{array}$ & 2016 & $\begin{array}{c}\text { success } \\
\text { measures }\end{array}$ & explicit \\
\hline 283 & $\begin{array}{l}\text { intangible benefits (for exam- } \\
\text { ple, improvement of compa- } \\
\text { ny's market image) }\end{array}$ & 5 & 25 & 5 & 25 & 7 & 33 & $\mathrm{PM}$ & $\begin{array}{l}\text { Varajão } \\
\text { and Trigo }\end{array}$ & 2016 & $\begin{array}{l}\text { success } \\
\text { measures }\end{array}$ & explicit \\
\hline
\end{tabular}




\begin{tabular}{|c|c|c|c|c|c|c|c|c|c|c|c|c|}
\hline 284 & $\begin{array}{l}\text { Adequacy of company-wide } \\
\text { education on the concepts of } \\
\text { risk management }\end{array}$ & 1 & 5 & 1 & 5 & 1 & 5 & PM & Cooke-Davis & 2002 & success & explicit \\
\hline 285 & $\begin{array}{l}\text { Adequacy with which a visible } \\
\text { risk register is maintained }\end{array}$ & 1 & 5 & 1 & 5 & 1 & 5 & $\mathrm{PM}$ & Cooke-Davis & 2002 & success & explicit \\
\hline 286 & $\begin{array}{l}\text { Adequacy of an up-to-date } \\
\text { risk management plan }\end{array}$ & 1 & 5 & 1 & 5 & 1 & 5 & PM & Cooke-Davis & 2002 & success & explicit \\
\hline 287 & $\begin{array}{l}\text { Allow changes to scope only } \\
\text { through a mature scope } \\
\text { change control process }\end{array}$ & 1 & 2 & 1 & 2 & 4 & 22 & $\mathrm{PM}$ & Cooke-Davis & 2002 & success & explicit \\
\hline 288 & $\begin{array}{l}\text { Keep project (or project stage } \\
\text { duration) as far below } 3 \text { years } \\
\text { as possible ( } 1 \text { year is better) }\end{array}$ & 4 & 23 & 4 & 23 & 4 & 23 & $\mathrm{PM}$ & Cooke-Davis & 2002 & success & explicit \\
\hline 289 & $\begin{array}{l}\text { Maintain the integrity of the } \\
\text { performance measurement } \\
\text { baseline }\end{array}$ & 4 & 21 & 4 & 22 & 4 & 22 & $\mathrm{PM}$ & Cooke-Davis & 2002 & success & explicit \\
\hline 290 & $\begin{array}{l}\text { A suite of project, programme } \\
\text { and portfolio metrics that pro- } \\
\text { vides direct "line of sight"' } \\
\text { feedback on current project } \\
\text { performance, and anticipated } \\
\text { future success, so that project, } \\
\text { portfolio and corporate deci- } \\
\text { sions can be aligned }\end{array}$ & 4 & 22 & 4 & 22 & 5 & 26 & $\mathrm{PM}$ & Cooke-Davis & 2002 & success & explicit \\
\hline 291 & $\begin{array}{l}\text { Adequacy of documentation of } \\
\text { organisational responsibilities } \\
\text { on the project }\end{array}$ & 3 & 11 & 1 & 5 & 3 & 11 & $\mathrm{PM}$ & Cooke-Davis & 2002 & success & explicit \\
\hline 292 & $\begin{array}{l}\text { An effective means of "learn- } \\
\text { ing from experience" on pro- } \\
\text { jects, that combines explicit } \\
\text { knowledge with tacit } \\
\text { knowledge in a way that en- } \\
\text { courages people to learn and } \\
\text { to embed that learning into } \\
\text { continuous improvement of } \\
\text { project management processes } \\
\text { and practices }\end{array}$ & 3 & 11 & 3 & 11 & 3 & 11 & $\mathrm{PM}$ & Cooke-Davis & 2002 & success & explicit \\
\hline 293 & $\begin{array}{l}\text { Portfolio- and programme } \\
\text { management practices that al- } \\
\text { low the enterprise to resource } \\
\text { fully a suite of projects that } \\
\text { are thoughtfully and dynami- } \\
\text { cally matched to the corporate } \\
\text { strategy and business objec- } \\
\text { tives }\end{array}$ & 5 & 26 & 5 & 26 & 5 & 26 & PM & Cooke-Davis & 2002 & success & explicit \\
\hline 294 & $\begin{array}{l}\text { Maturity of an organisation's } \\
\text { processes for assigning owner- } \\
\text { ship of risks }\end{array}$ & 6 & 28 & 6 & 28 & 6 & 28 & PM & Cooke-Davis & 2002 & success & explicit \\
\hline 295 & $\begin{array}{l}\text { The existence of an effective } \\
\text { benefits delivery and manage- } \\
\text { ment process that involves the } \\
\text { mutual co-operation of project } \\
\text { management and line manage- } \\
\text { ment functions }\end{array}$ & 6 & 28 & 6 & 28 & 6 & 28 & PM & Cooke-Davis & 2002 & success & explicit \\
\hline 296 & $\begin{array}{l}\text { using project plans as living } \\
\text { documents }\end{array}$ & 1 & 3 & 1 & 3 & 3 & 11 & PM & $\begin{array}{l}\text { Jugdev } \\
\text { and Müller }\end{array}$ & 2005 & success & implicit \\
\hline 297 & change management & 1 & 2 & 1 & 2 & 1 & 2 & $\mathrm{PM}$ & $\begin{array}{l}\text { Jugdev } \\
\text { and Müller }\end{array}$ & 2005 & success & implicit \\
\hline 298 & agile requirements & 1 & 3 & 1 & 3 & 5 & 25 & $\mathrm{PM}$ & $\begin{array}{l}\text { Jugdev } \\
\text { and Müller }\end{array}$ & 2005 & success & implicit \\
\hline
\end{tabular}




\begin{tabular}{|c|c|c|c|c|c|c|c|c|c|c|c|c|}
\hline 299 & $\begin{array}{l}\text { The project manager should be } \\
\text { empowered with flexibility to } \\
\text { deal with unforeseen circum- } \\
\text { stances as they see best, and } \\
\text { with the owner giving guid- } \\
\text { ance as to how they think the } \\
\text { project should be best } \\
\text { achieved }\end{array}$ & 1 & 3 & 1 & 3 & 1 & 3 & PM & $\begin{array}{l}\text { Jugdev } \\
\text { and Müller }\end{array}$ & 2005 & success & explicit \\
\hline 300 & project scope is managed & 4 & 17 & 5 & 25 & 4 & 23 & $\mathrm{PM}$ & $\begin{array}{l}\text { Jugdev } \\
\text { and Müller }\end{array}$ & 2005 & success & implicit \\
\hline 301 & team & 4 & 24 & 4 & 24 & 4 & 24 & PM & $\begin{array}{l}\text { Jugdev } \\
\text { and Müller }\end{array}$ & 2005 & success & implicit \\
\hline 302 & $\begin{array}{l}\text { project manager selection cri- } \\
\text { teria }\end{array}$ & 4 & 24 & 4 & 24 & 4 & 24 & $\mathrm{PM}$ & $\begin{array}{l}\text { Jugdev } \\
\text { and Müller }\end{array}$ & 2005 & success & implicit \\
\hline 303 & $\begin{array}{l}\text { project manager leadership } \\
\text { style }\end{array}$ & 4 & 17 & 4 & 17 & 4 & 17 & $\mathrm{PM}$ & $\begin{array}{l}\text { Jugdev } \\
\text { and Müller }\end{array}$ & 2005 & success & implicit \\
\hline 304 & commitment to planning & 4 & 23 & 4 & 23 & 4 & 23 & $\mathrm{PM}$ & $\begin{array}{l}\text { Jugdev } \\
\text { and Müller }\end{array}$ & 2005 & success & implicit \\
\hline 305 & commitment to control & 4 & 21 & 4 & 21 & 4 & 21 & PM & $\begin{array}{l}\text { Jugdev } \\
\text { and Müller }\end{array}$ & 2005 & success & implicit \\
\hline 306 & Project schedule/plan & 4 & 23 & 4 & 23 & 4 & 23 & PM & $\begin{array}{l}\text { Jugdev } \\
\text { and Müller }\end{array}$ & 2005 & success & explicit \\
\hline 307 & Personnel & 4 & 24 & 4 & 24 & 3 & 9 & $\mathrm{PM}$ & $\begin{array}{l}\text { Jugdev } \\
\text { and Müller }\end{array}$ & 2005 & success & explicit \\
\hline 308 & Monitoring and feedback & 4 & 22 & 4 & 22 & 3 & 11 & $\mathrm{PM}$ & $\begin{array}{l}\text { Jugdev } \\
\text { and Müller }\end{array}$ & 2005 & success & explicit \\
\hline 309 & Troubleshooting Expertise & 4 & 17 & 1 & 5 & 4 & 19 & PM & $\begin{array}{l}\text { Jugdev } \\
\text { and Müller }\end{array}$ & 2005 & success & explicit \\
\hline 310 & experienced project managers & 4 & 17 & 4 & 24 & 4 & 17 & PM & $\begin{array}{l}\text { Jugdev } \\
\text { and Müller }\end{array}$ & 2005 & success & implicit \\
\hline 311 & minimizing scope & 4 & 23 & 4 & 23 & 4 & 23 & PM & $\begin{array}{l}\text { Jugdev } \\
\text { and Müller }\end{array}$ & 2005 & success & implicit \\
\hline 312 & formal methodology & 4 & 23 & 4 & 23 & 4 & 23 & $\mathrm{PM}$ & $\begin{array}{l}\text { Jugdev } \\
\text { and Müller }\end{array}$ & 2005 & success & implicit \\
\hline 313 & reliable estimates & 4 & 23 & 4 & 23 & 4 & 23 & PM & $\begin{array}{l}\text { Jugdev } \\
\text { and Müller }\end{array}$ & 2005 & success & implicit \\
\hline 314 & skilled staff & 4 & 16 & 4 & 24 & 4 & 16 & PM & $\begin{array}{l}\text { Jugdev } \\
\text { and Müller }\end{array}$ & 2005 & success & implicit \\
\hline 315 & $\begin{array}{l}\text { process improvement using } \\
\text { software development capabil- } \\
\text { ity maturity models }\end{array}$ & 4 & 19 & 4 & 19 & 4 & 19 & PM & $\begin{array}{l}\text { Jugdev } \\
\text { and Müller }\end{array}$ & 2005 & success & implicit \\
\hline 316 & client satisfaction & 2 & 6 & 3 & 8 & 2 & 6 & PM & $\begin{array}{l}\text { Jugdev } \\
\text { and Müller }\end{array}$ & 2005 & success & implicit \\
\hline 317 & Customer satisfaction & 2 & 6 & 2 & 6 & 2 & 6 & PM & $\begin{array}{l}\text { Jugdev } \\
\text { and Müller }\end{array}$ & 2005 & success & implicit \\
\hline 318 & Client acceptance & 2 & 6 & 3 & 8 & 3 & 8 & PM & $\begin{array}{l}\text { Jugdev } \\
\text { and Müller }\end{array}$ & 2005 & success & explicit \\
\hline 319 & regenerative projects & 2 & 7 & 4 & 23 & 3 & 11 & PM & $\begin{array}{l}\text { Jugdev } \\
\text { and Müller }\end{array}$ & 2005 & success & implicit \\
\hline 320 & management support & 3 & 15 & 3 & 15 & 3 & 15 & $\mathrm{PM}$ & $\begin{array}{l}\text { Jugdev } \\
\text { and Müller }\end{array}$ & 2005 & success & implicit \\
\hline 321 & stakeholder satisfaction & 3 & 12 & 3 & 12 & 2 & 6 & $\mathrm{PM}$ & $\begin{array}{l}\text { Jugdev } \\
\text { and Müller }\end{array}$ & 2005 & success & implicit \\
\hline 322 & staff training and education & 3 & 11 & 3 & 11 & 3 & 11 & $\mathrm{PM}$ & $\begin{array}{l}\text { Jugdev } \\
\text { and Müller }\end{array}$ & 2005 & success & implicit \\
\hline 323 & dedicated resources & 3 & 13 & 3 & 13 & 3 & 13 & PM & $\begin{array}{l}\text { Jugdev } \\
\text { and Müller }\end{array}$ & 2005 & success & implicit \\
\hline
\end{tabular}




\begin{tabular}{|c|c|c|c|c|c|c|c|c|c|c|c|c|}
\hline 324 & $\begin{array}{l}\text { strong leadership and manage- } \\
\text { ment }\end{array}$ & 3 & 15 & 3 & 15 & 6 & 28 & PM & $\begin{array}{l}\text { Jugdev } \\
\text { and Müller }\end{array}$ & 2005 & success & implicit \\
\hline 325 & $\begin{array}{l}\text { concurrent development of the } \\
\text { individual }\end{array}$ & 3 & 11 & 3 & 11 & 3 & 9 & PM & \begin{tabular}{|l} 
Jugdev \\
and Müller
\end{tabular} & 2005 & success & implicit \\
\hline 326 & effective communication & 3 & 10 & 3 & 10 & 3 & 10 & PM & \begin{tabular}{|l} 
Jugdev \\
and Müller
\end{tabular} & 2005 & success & implicit \\
\hline 327 & $\begin{array}{l}\text { corporate understanding of } \\
\text { project management by every- } \\
\text { one involved }\end{array}$ & 3 & 11 & 3 & 11 & 3 & 11 & PM & $\begin{array}{l}\text { Jugdev } \\
\text { and Müller }\end{array}$ & 2005 & success & implicit \\
\hline 328 & $\begin{array}{l}\text { executive commitment to pro- } \\
\text { ject management }\end{array}$ & 3 & 15 & 3 & 15 & 3 & 15 & PM & \begin{tabular}{|l|} 
Jugdev \\
and Müller
\end{tabular} & 2005 & success & implicit \\
\hline 329 & Top management support & 3 & 15 & 3 & 15 & 3 & 15 & PM & \begin{tabular}{|l} 
Jugdev \\
and Müller
\end{tabular} & 2005 & success & explicit \\
\hline 330 & Client consultation & 3 & 8 & 3 & 8 & 3 & 8 & PM & \begin{tabular}{|l} 
Jugdev \\
and Müller
\end{tabular} & 2005 & success & explicit \\
\hline 331 & Channels of Communication & 3 & 10 & 3 & 10 & 3 & 10 & PM & $\begin{array}{l}\text { Jugdev } \\
\text { and Müller }\end{array}$ & 2005 & success & explicit \\
\hline 332 & user involvement & 3 & 8 & 3 & 8 & 3 & 8 & $\mathrm{PM}$ & $\begin{array}{l}\text { Jugdev } \\
\text { and Müller }\end{array}$ & 2005 & success & implicit \\
\hline 333 & $\begin{array}{l}\text { executive management sup- } \\
\text { port }\end{array}$ & 3 & 15 & 3 & 15 & 3 & 15 & $\mathrm{PM}$ & $\begin{array}{l}\text { Jugdev } \\
\text { and Müller }\end{array}$ & 2005 & success & implicit \\
\hline 334 & $\begin{array}{l}\text { Communication the the right } \\
\text { level }\end{array}$ & 3 & 10 & 3 & 10 & 3 & 10 & PM & \begin{tabular}{|l} 
Jugdev \\
and Müller
\end{tabular} & 2005 & success & implicit \\
\hline 335 & $\begin{array}{l}\text { Communication with the right } \\
\text { people }\end{array}$ & 3 & 10 & 3 & 10 & 3 & 10 & PM & \begin{tabular}{|l} 
Jugdev \\
and Müller
\end{tabular} & 2005 & success & implicit \\
\hline 336 & $\begin{array}{l}\text { A collaborative working rela- } \\
\text { tionship should be maintained } \\
\text { between the project owner (or } \\
\text { sponsor) and project manager, } \\
\text { with both viewing the project } \\
\text { as a partnership }\end{array}$ & 3 & 12 & 4 & 24 & 3 & 11 & PM & $\begin{array}{l}\text { Jugdev } \\
\text { and Müller }\end{array}$ & 2005 & success & explicit \\
\hline 337 & clear mission & 5 & 25 & 5 & 25 & 6 & 28 & PM & \begin{tabular}{|l|} 
Jugdev \\
and Müller
\end{tabular} & 2005 & success & implicit \\
\hline 338 & clear objective and scope & 5 & 25 & 5 & 25 & 5 & 25 & PM & \begin{tabular}{|l|} 
Jugdev \\
and Müller
\end{tabular} & 2005 & success & implicit \\
\hline 339 & $\begin{array}{l}\text { dividing the project into man- } \\
\text { ageable components }\end{array}$ & 5 & 25 & 4 & 23 & 4 & 23 & $\mathrm{PM}$ & \begin{tabular}{|l|l} 
Jugdev \\
and Müller
\end{tabular} & 2005 & success & implicit \\
\hline 340 & $\begin{array}{l}\text { alignment between project } \\
\text { management and strategic } \\
\text { management }\end{array}$ & 5 & 26 & 5 & 26 & 5 & 26 & PM & $\begin{array}{l}\text { Jugdev } \\
\text { and Müller }\end{array}$ & 2005 & success & implicit \\
\hline 341 & Project mission & 5 & 25 & 5 & 25 & 4 & 23 & PM & \begin{tabular}{|l|} 
Jugdev \\
and Müller
\end{tabular} & 2005 & success & explicit \\
\hline 342 & clear business objectives & 5 & 25 & 5 & 25 & 5 & 25 & PM & \begin{tabular}{|l|} 
Jugdev \\
and Müller
\end{tabular} & 2005 & success & implicit \\
\hline 343 & strategically managed projects & 5 & 26 & 5 & 26 & 5 & 26 & PM & \begin{tabular}{|l|} 
Jugdev \\
and Müller
\end{tabular} & 2005 & success & implicit \\
\hline 344 & aligned projects & 5 & 26 & 5 & 26 & 5 & 26 & PM & $\begin{array}{l}\text { Jugdev } \\
\text { and Müller }\end{array}$ & 2005 & success & implicit \\
\hline 345 & $\begin{array}{l}\text { projects that involve transi- } \\
\text { tional management }\end{array}$ & 5 & 26 & 5 & 26 & 1 & 3 & PM & $\begin{array}{l}\text { Jugdev } \\
\text { and Müller }\end{array}$ & 2005 & success & implicit \\
\hline 346 & $\begin{array}{l}\text { Success criteria should be } \\
\text { agreed on the stakeholders be- } \\
\text { fore the start of the project, } \\
\text { and repeatedly at configura- } \\
\text { tion review points thoughout } \\
\text { the project }\end{array}$ & 5 & 25 & 4 & 23 & 3 & 8 & PM & $\begin{array}{l}\text { Jugdev } \\
\text { and Müller }\end{array}$ & 2005 & success & explicit \\
\hline
\end{tabular}




\begin{tabular}{|c|c|c|c|c|c|c|c|c|c|c|c|c|}
\hline 347 & $\begin{array}{l}\text { The owner should take an in- } \\
\text { terest in the performance of } \\
\text { the project }\end{array}$ & 5 & 26 & 3 & 14 & 3 & 15 & PM & $\begin{array}{l}\text { Jugdev } \\
\text { and Müller }\end{array}$ & 2005 & success & explicit \\
\hline 348 & $\begin{array}{l}\text { understanding of project man- } \\
\text { agement as a strategic asset }\end{array}$ & 5 & 26 & 5 & 26 & 4 & 17 & PM & $\begin{array}{l}\text { Jugdev } \\
\text { and Müller }\end{array}$ & 2005 & success & implicit \\
\hline 349 & organization & 6 & 28 & 6 & 28 & 6 & 28 & PM & $\begin{array}{l}\text { Jugdev } \\
\text { and Müller }\end{array}$ & 2005 & success & implicit \\
\hline 350 & organizational effectiveness & 6 & 28 & 6 & 28 & 6 & 28 & PM & $\begin{array}{l}\text { Jugdev } \\
\text { and Müller }\end{array}$ & 2005 & success & implicit \\
\hline 351 & organizational adaptability & 6 & 28 & 6 & 28 & 6 & 28 & PM & $\begin{array}{l}\text { Jugdev } \\
\text { and Müller }\end{array}$ & 2005 & success & implicit \\
\hline 352 & $\begin{array}{l}\text { standard software infrastruc- } \\
\text { ture }\end{array}$ & 6 & 27 & 6 & 27 & 6 & 27 & PM & $\begin{array}{l}\text { Jugdev } \\
\text { and Müller }\end{array}$ & 2005 & success & implicit \\
\hline 353 & good tools & 7 & 32 & 7 & 32 & 7 & 32 & PM & $\begin{array}{l}\text { Jugdev } \\
\text { and Müller }\end{array}$ & 2005 & success & implicit \\
\hline 354 & $\begin{array}{l}\text { Technology to Support the } \\
\text { Project }\end{array}$ & 7 & 33 & 7 & 33 & 7 & 33 & PM & $\begin{array}{l}\text { Jugdev } \\
\text { and Müller }\end{array}$ & 2005 & success & explicit \\
\hline 355 & $\begin{array}{l}\text { Assisted in early trouble- } \\
\text { shooting }\end{array}$ & 1 & 5 & 1 & 5 & 1 & 5 & PM & Dvir et al. & 1998 & success & explicit \\
\hline 356 & Encouraging new ideas & 1 & 2 & 1 & 2 & 1 & 2 & PM & Dvir et al. & 1998 & success & explicit \\
\hline 357 & $\begin{array}{l}\text { Willingness to consider } \\
\text { changes and new approaches }\end{array}$ & 1 & 3 & 1 & 3 & 1 & 2 & PM & Dvir et al. & 1998 & success & explicit \\
\hline 358 & $\begin{array}{l}\text { Criteria for operational effec- } \\
\text { tiveness }\end{array}$ & 4 & 21 & 4 & 21 & 4 & 22 & PM & Dvir et al. & 1998 & success & explicit \\
\hline 359 & Method for use in battle & 4 & 23 & 4 & 23 & 4 & 23 & $\mathrm{PM}$ & Dvir et al. & 1998 & success & explicit \\
\hline 360 & $\begin{array}{l}\text { Check for existence of alterna- } \\
\text { tives }\end{array}$ & 4 & 23 & 4 & 23 & 1 & 2 & PM & Dvir et al. & 1998 & success & explicit \\
\hline 361 & Operational specifications & 4 & 23 & 4 & 23 & 4 & 23 & PM & Dvir et al. & 1998 & success & explicit \\
\hline 362 & Reliability specifications & 4 & 22 & 4 & 23 & 4 & 23 & PM & Dvir et al. & 1998 & success & explicit \\
\hline 363 & Project plan & 4 & 23 & 4 & 23 & 4 & 23 & PM & Dvir et al. & 1998 & success & explicit \\
\hline 364 & $\begin{array}{l}\text { Cost estimation for the entire } \\
\text { project }\end{array}$ & 4 & 23 & 4 & 23 & 3 & 13 & PM & Dvir et al. & 1998 & success & explicit \\
\hline 365 & $\begin{array}{l}\text { Negotiations with alternative } \\
\text { contractors }\end{array}$ & 4 & 23 & 4 & 23 & 3 & 12 & PM & Dvir et al. & 1998 & success & explicit \\
\hline 366 & Detailed payment milestones & 4 & 21 & 4 & 23 & 4 & 23 & $\mathrm{PM}$ & Dvir et al. & 1998 & success & explicit \\
\hline 367 & $\begin{array}{l}\text { Key personnel stayed through- } \\
\text { out the project }\end{array}$ & 4 & 24 & 4 & 24 & 3 & 9 & PM & Dvir et al. & 1998 & success & explicit \\
\hline 368 & $\begin{array}{l}\text { Overall responsibility for pro- } \\
\text { ject success }\end{array}$ & 4 & 22 & 3 & 14 & 6 & 28 & PM & Dvir et al. & 1998 & success & explicit \\
\hline 369 & $\begin{array}{l}\text { High professionell qualifica- } \\
\text { tions }\end{array}$ & 4 & 24 & 4 & 24 & 4 & 16 & PM & Dvir et al. & 1998 & success & explicit \\
\hline 370 & $\begin{array}{l}\text { Organizational and logistic } \\
\text { preparations }\end{array}$ & 4 & 23 & 4 & 23 & 6 & 28 & PM & Dvir et al. & 1998 & success & explicit \\
\hline 371 & Risk management & 4 & 17 & 1 & 5 & 1 & 5 & $\mathrm{PM}$ & Dvir et al. & 1998 & success & explicit \\
\hline 372 & $\begin{array}{l}\text { Resources and schedule con- } \\
\text { trol }\end{array}$ & 4 & 21 & 4 & 21 & 4 & 22 & PM & Dvir et al. & 1998 & success & explicit \\
\hline 373 & $\begin{array}{l}\text { Quality and reliability assur- } \\
\text { ance }\end{array}$ & 4 & 21 & 4 & 21 & 4 & 22 & PM & Dvir et al. & 1998 & success & explicit \\
\hline 374 & Test management & 4 & 19 & 4 & 23 & 4 & 17 & PM & Dvir et al. & 1998 & success & explicit \\
\hline 375 & Personnel management & 4 & 24 & 4 & 24 & 4 & 17 & PM & Dvir et al. & 1998 & success & explicit \\
\hline 376 & Decision-making procedures & 4 & 17 & 4 & 17 & 6 & 28 & PM & Dvir et al. & 1998 & success & explicit \\
\hline 377 & Conceptual prototype & 4 & 23 & 4 & 23 & 4 & 23 & PM & Dvir et al. & 1998 & success & explicit \\
\hline 378 & Prototype of field tests & 4 & 23 & 4 & 23 & 4 & 23 & PM & Dvir et al. & 1998 & success & explicit \\
\hline 379 & For the whole system & 4 & 19 & 4 & 19 & 4 & 19 & PM & Dvir et al. & 1998 & success & explicit \\
\hline 380 & Producibility & 4 & 20 & 4 & 20 & 4 & 20 & PM & Dvir et al. & 1998 & success & explicit \\
\hline
\end{tabular}




\begin{tabular}{|c|c|c|c|c|c|c|c|c|c|c|c|c|}
\hline 381 & Maintainability & 4 & 20 & 4 & 20 & 4 & 20 & PM & Dvir et al. & 1998 & success & explicit \\
\hline 382 & Quality and reliability & 4 & 20 & 4 & 20 & 4 & 20 & PM & Dvir et al. & 1998 & success & explicit \\
\hline 383 & Human engineering & 4 & 16 & 4 & 23 & 4 & 16 & PM & Dvir et al. & 1998 & success & explicit \\
\hline 384 & Final system requirements & 4 & 23 & 4 & 23 & 4 & 23 & $\mathrm{PM}$ & Dvir et al. & 1998 & success & explicit \\
\hline 385 & System concept & 4 & 23 & 4 & 23 & 4 & 23 & PM & Dvir et al. & 1998 & success & explicit \\
\hline 386 & System configuration & 4 & 16 & 4 & 16 & 4 & 16 & PM & Dvir et al. & 1998 & success & explicit \\
\hline 387 & Subsystem specifications & 4 & 23 & 4 & 23 & 4 & 23 & PM & Dvir et al. & 1998 & success & explicit \\
\hline 388 & Presentation of prototype & 4 & 23 & 4 & 23 & 4 & 23 & PM & Dvir et al. & 1998 & success & explicit \\
\hline 389 & Design freeze & 4 & 19 & 4 & 23 & 4 & 23 & PM & Dvir et al. & 1998 & success & explicit \\
\hline 390 & Qualification tests & 4 & 22 & 4 & 22 & 4 & 22 & PM & Dvir et al. & 1998 & success & explicit \\
\hline 391 & Final test and delivery & 4 & 22 & 4 & 22 & 4 & 22 & PM & Dvir et al. & 1998 & success & explicit \\
\hline 392 & Schedule and milestones & 4 & 21 & 4 & 23 & 4 & 22 & PM & Dvir et al. & 1998 & success & explicit \\
\hline 393 & Configuration control & 4 & 21 & 4 & 23 & 4 & 22 & PM & Dvir et al. & 1998 & success & explicit \\
\hline 394 & $\begin{array}{l}\text { Used as a tool for senior man- } \\
\text { agement }\end{array}$ & 4 & 22 & 3 & 15 & 4 & 22 & $\mathrm{PM}$ & Dvir et al. & 1998 & success & explicit \\
\hline 395 & Day-to-day follow-up & 4 & 22 & 4 & 22 & 4 & 22 & PM & Dvir et al. & 1998 & success & explicit \\
\hline 396 & $\begin{array}{l}\text { Personal supervision of perfor- } \\
\text { mance }\end{array}$ & 4 & 17 & 4 & 24 & 4 & 17 & PM & Dvir et al. & 1998 & success & explicit \\
\hline 397 & Involvement with workers & 4 & 17 & 4 & 17 & 3 & 11 & PM & Dvir et al. & 1998 & success & explicit \\
\hline 398 & $\begin{array}{l}\text { Involving workers in decision- } \\
\text { making }\end{array}$ & 4 & 17 & 4 & 17 & 3 & 11 & $\mathrm{PM}$ & Dvir et al. & 1998 & success & explicit \\
\hline 399 & Frequent updating of status & 4 & 22 & 3 & 11 & 3 & 11 & $\mathrm{PM}$ & Dvir et al. & 1998 & success & explicit \\
\hline 400 & $\begin{array}{l}\text { Key personnel in the project } \\
\text { for its entire duration }\end{array}$ & 4 & 24 & 4 & 24 & 4 & 24 & $\mathrm{PM}$ & Dvir et al. & 1998 & success & explicit \\
\hline 401 & $\begin{array}{l}\text { Key personnel with strong } \\
\text { managerial qualifications }\end{array}$ & 4 & 17 & 4 & 24 & 4 & 24 & PM & Dvir et al. & 1998 & success & explicit \\
\hline 402 & $\begin{array}{l}\text { Some team members with op- } \\
\text { erational experience }\end{array}$ & 4 & 24 & 4 & 24 & 4 & 24 & $\mathrm{PM}$ & Dvir et al. & 1998 & success & explicit \\
\hline 403 & Professionally experienced & 4 & 17 & 4 & 17 & 4 & 17 & $\mathrm{PM}$ & Dvir et al. & 1998 & success & explicit \\
\hline 404 & $\begin{array}{l}\text { Extensive managerial experi- } \\
\text { ence }\end{array}$ & 4 & 17 & 4 & 24 & 4 & 17 & PM & Dvir et al. & 1998 & success & explicit \\
\hline 405 & $\begin{array}{l}\text { Recognition of need by end- } \\
\text { user }\end{array}$ & 2 & 6 & 2 & 6 & 3 & 8 & PM & Dvir et al. & 1998 & success & explicit \\
\hline 406 & Detailed contract documents & 2 & 7 & 4 & 23 & 3 & 12 & PM & Dvir et al. & 1998 & success & explicit \\
\hline 407 & Specifications & 2 & 7 & 4 & 23 & 4 & 22 & PM & Dvir et al. & 1998 & success & explicit \\
\hline 408 & $\begin{array}{l}\text { level of authority entrusted to } \\
\text { the project manager }\end{array}$ & 3 & 15 & 4 & 24 & 6 & 28 & $\mathrm{PM}$ & Dvir et al. & 1998 & success & implicit \\
\hline 409 & $\begin{array}{l}\text { integration of numerous man- } \\
\text { agement functions }\end{array}$ & 3 & 15 & 3 & 15 & 6 & 28 & PM & Dvir et al. & 1998 & success & implicit \\
\hline 410 & $\begin{array}{l}\text { Project acknowledged as being } \\
\text { urgent }\end{array}$ & 3 & 15 & 3 & 15 & 4 & 23 & PM & Dvir et al. & 1998 & success & explicit \\
\hline 411 & $\begin{array}{l}\text { Team includes end-user repre- } \\
\text { sentatives }\end{array}$ & 3 & 8 & 4 & 24 & 4 & 24 & PM & Dvir et al. & 1998 & success & explicit \\
\hline 412 & $\begin{array}{l}\text { Active participation in devel- } \\
\text { opment activities }\end{array}$ & 3 & 8 & 3 & 8 & 3 & 8 & PM & Dvir et al. & 1998 & success & explicit \\
\hline 413 & $\begin{array}{l}\text { Main contractor involved in } \\
\text { system definition }\end{array}$ & 3 & 10 & 3 & 10 & 3 & 12 & PM & Dvir et al. & 1998 & success & explicit \\
\hline 414 & Communication with customer & 3 & 8 & 3 & 8 & 3 & 8 & PM & Dvir et al. & 1998 & success & explicit \\
\hline 415 & Communication and reports & 3 & 11 & 3 & 11 & 3 & 11 & PM & Dvir et al. & 1998 & success & explicit \\
\hline 416 & Design to cost & 3 & 13 & 3 & 13 & 3 & 13 & PM & Dvir et al. & 1998 & success & explicit \\
\hline 417 & Budget utilization & 3 & 13 & 4 & 23 & 4 & 22 & PM & Dvir et al. & 1998 & success & explicit \\
\hline 418 & Profit and loss report & 3 & 11 & 3 & 11 & 3 & 11 & PM & Dvir et al. & 1998 & success & explicit \\
\hline 419 & Cash-flow report & 3 & 11 & 3 & 11 & 3 & 11 & PM & Dvir et al. & 1998 & success & explicit \\
\hline
\end{tabular}




\begin{tabular}{|c|c|c|c|c|c|c|c|c|c|c|c|c|}
\hline 420 & With all subcontractors & 3 & 10 & 3 & 10 & 3 & 10 & PM & Dvir et al. & 1998 & success & explicit \\
\hline 421 & To higher management & 3 & 11 & 3 & 11 & 3 & 11 & PM & Dvir et al. & 1998 & success & explicit \\
\hline 422 & Existence of unit spirit & 3 & 14 & 3 & 14 & 6 & 28 & PM & Dvir et al. & 1998 & uccess & explicit \\
\hline 423 & $\begin{array}{l}\text { Social activities out of work- } \\
\text { ing hours }\end{array}$ & 3 & 14 & 3 & 14 & 3 & 14 & PM & Dvir et al. & 1998 & success & explicit \\
\hline 424 & Room for professional growth & 3 & 11 & 3 & 11 & 3 & 11 & PM & Dvir et al. & 1998 & success & explicit \\
\hline 425 & \begin{tabular}{|l|} 
Possibilities for consulting \\
with experienced professionals
\end{tabular} & 3 & 10 & 3 & 10 & 3 & 11 & PM & Dvir et al. & 1998 & success & explicit \\
\hline 426 & $\begin{array}{l}\text { Acts to increase workers' mo- } \\
\text { tivation }\end{array}$ & 3 & 14 & 3 & 14 & 3 & 14 & PM & Dvir et al. & 1998 & success & explicit \\
\hline 427 & Open communication & 3 & 10 & 3 & 10 & 3 & 10 & $\mathrm{PM}$ & Dvir et al. & 1998 & success & explicit \\
\hline 428 & $\begin{array}{l}\text { Involvement of manager in } \\
\text { day-to-day problem solving }\end{array}$ & 3 & 11 & 3 & 11 & 3 & 11 & PM & Dvir et al. & 1998 & success & explicit \\
\hline 429 & $\begin{array}{l}\text { Application of lessons learned } \\
\text { during project execution }\end{array}$ & 3 & 11 & 3 & 11 & 3 & 11 & PM & Dvir et al. & 1998 & success & explicit \\
\hline 430 & $\begin{array}{l}\text { Detailed operational require- } \\
\text { ments }\end{array}$ & 5 & 25 & 5 & 25 & 5 & 25 & PM & Dvir et al. & 1998 & success & explicit \\
\hline 431 & Technical specifications & 5 & 25 & 4 & 23 & 7 & 29 & PM & Dvir et al. & 1998 & success & explicit \\
\hline 432 & Criteria for acceptance & 5 & 25 & 4 & 23 & 2 & 6 & PM & Dvir et al. & 1998 & success & explicit \\
\hline 433 & Exact specification of tasks & 5 & 25 & 5 & 25 & 3 & 11 & PM & Dvir et al. & 1998 & success & explicit \\
\hline 434 & $\begin{array}{l}\text { Definition of organizational } \\
\text { structure }\end{array}$ & 6 & 28 & 4 & 23 & 6 & 28 & PM & Dvir et al. & 1998 & success & explicit \\
\hline 435 & $\begin{array}{l}\text { Type of organizational struc- } \\
\text { ture }\end{array}$ & 6 & 28 & 4 & 23 & 6 & 28 & PM & Dvir et al. & 1998 & success & explicit \\
\hline 436 & Fit of organizational structure & 6 & 28 & 4 & 23 & 6 & 28 & PM & al. & 1998 & & xplicit \\
\hline 437 & \begin{tabular}{|l|} 
Infrastructure from earlier pro- \\
jects
\end{tabular} & 6 & 27 & 6 & 27 & 6 & 27 & PM & Dvir et al. & 1998 & success & explicit \\
\hline 438 & $\begin{array}{l}\text { Acquired from external } \\
\text { sources }\end{array}$ & 6 & 27 & 7 & 32 & 7 & 33 & PM & Dvir et al. & 1998 & success & explicit \\
\hline 439 & System integration & 6 & 27 & 6 & 27 & 6 & 27 & PM & & 1998 & success & explicit \\
\hline 440 & $\begin{array}{l}\text { Planning of activities with } \\
\text { Work Breakdown Structure }\end{array}$ & 6 & 27 & 4 & 23 & 4 & 23 & PM & Dvir et al. & 1998 & success & explicit \\
\hline 441 & Managers as role models & 6 & 28 & 6 & 28 & 6 & 28 & PM & Dvir et al. & 1998 & success & explicit \\
\hline 442 & $\begin{array}{l}\text { Setting general policy and } \\
\text { goals }\end{array}$ & 6 & 28 & 6 & 28 & 5 & 25 & PM & Dvir et al. & 1998 & success & explicit \\
\hline 443 & $\begin{array}{l}\text { Higher operational value than } \\
\text { other systems }\end{array}$ & 7 & 33 & 6 & 27 & 2 & 6 & $\mathrm{PM}$ & Dvir et al. & 1998 & success & explicit \\
\hline 444 & Technical feasibility checked & 7 & 32 & 4 & 23 & 7 & 32 & $\mathrm{PM}$ & Dvir et al. & 1998 & success & explicit \\
\hline 445 & $\begin{array}{l}\text { Alternative technical solution } \\
\text { checked }\end{array}$ & 7 & 32 & 4 & 23 & 7 & 32 & PM & Dvir et al. & 1998 & success & explicit \\
\hline 446 & $\begin{array}{l}\text { Proposal based on existing } \\
\text { technological infrastructure }\end{array}$ & 7 & 33 & 4 & 23 & 6 & 27 & PM & Dvir et al. & 1998 & success & explicit \\
\hline 447 & $\begin{array}{l}\text { Developed during the project's } \\
\text { execution }\end{array}$ & 7 & 33 & 7 & 33 & 6 & 27 & $\mathrm{PM}$ & Dvir et al. & 1998 & success & explicit \\
\hline 448 & Technical issues managed & 7 & 29 & 7 & 30 & 7 & 29 & PM & Dvir et al. & 1998 & success & xplicit \\
\hline 449 & $\begin{array}{l}\text { Constant follow-up of techno- } \\
\text { logical developments }\end{array}$ & 7 & 33 & 7 & 33 & 3 & 11 & $\mathrm{PM}$ & Dvir et al. & 1998 & success & explicit \\
\hline 450 & High technical level & 7 & 29 & 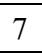 & 29 & 7 & 29 & $\mathrm{PM}$ & Dvir et al. & 1998 & success & explicit \\
\hline 451 & A technical leader & 7 & 30 & 7 & 30 & 7 & 30 & $\mathrm{PM}$ & Dvir et al. & 1998 & success & explicit \\
\hline 452 & $\begin{array}{l}\text { Revising reward and motiva- } \\
\text { tion systems }\end{array}$ & 1 & 2 & 1 & 2 & 1 & 2 & BPR & $\begin{array}{l}\text { Al-Mashari } \\
\text { and Zairi }\end{array}$ & 1999 & success & explicit \\
\hline 453 & $\begin{array}{l}\text { Creating an effective culture } \\
\text { for organisational change }\end{array}$ & 1 & 2 & 1 & 2 & 1 & 2 & BPR & $\begin{array}{l}\text { Al-Mashari } \\
\text { and Zairi }\end{array}$ & 1999 & success & explicit \\
\hline
\end{tabular}




\begin{tabular}{|c|c|c|c|c|c|c|c|c|c|c|c|c|}
\hline 454 & $\begin{array}{l}\text { Stimulating the organisation's } \\
\text { receptiveness to change }\end{array}$ & 1 & 2 & 1 & 2 & 1 & 2 & $\mathrm{BPR}$ & $\begin{array}{l}\text { Al-Mashari } \\
\text { and Zairi }\end{array}$ & 1999 & success & explicit \\
\hline 455 & $\begin{array}{l}\text { Lack of organisational readi- } \\
\text { ness for change }\end{array}$ & 1 & 2 & 1 & 2 & 1 & 2 & $\mathrm{BPR}$ & $\begin{array}{l}\text { Al-Mashari } \\
\text { and Zairi }\end{array}$ & 1999 & failure & explicit \\
\hline 456 & $\begin{array}{l}\text { Problems related to creating a } \\
\text { culture for change }\end{array}$ & 1 & 2 & 1 & 2 & 1 & 2 & $\mathrm{BPR}$ & $\begin{array}{l}\text { Al-Mashari } \\
\text { and Zairi }\end{array}$ & 1999 & failure & explicit \\
\hline 457 & effective management of risks & 4 & 17 & 1 & 5 & 1 & 5 & $\mathrm{BPR}$ & $\begin{array}{l}\text { Al-Mashari } \\
\text { and Zairi }\end{array}$ & 1999 & success & explicit \\
\hline 458 & Effective BPR teams & 4 & 24 & 4 & 24 & 4 & 24 & $\mathrm{BPR}$ & $\begin{array}{l}\text { Al-Mashari } \\
\text { and Zairi }\end{array}$ & 1999 & success & explicit \\
\hline 459 & $\begin{array}{l}\text { Effective planning and use of } \\
\text { project management tech- } \\
\text { niques }\end{array}$ & 4 & 23 & 4 & 23 & 4 & 17 & $\mathrm{BPR}$ & $\begin{array}{l}\text { Al-Mashari } \\
\text { and Zairi }\end{array}$ & 1999 & success & explicit \\
\hline 460 & $\begin{array}{l}\text { Appropriate use of methodol- } \\
\text { ogy }\end{array}$ & 4 & 23 & 4 & 23 & 4 & 23 & BPR & $\begin{array}{l}\text { Al-Mashari } \\
\text { and Zairi }\end{array}$ & 1999 & success & explicit \\
\hline 461 & Effective process redesign & 4 & 19 & 4 & 19 & 4 & 19 & $\mathrm{BPR}$ & $\begin{array}{l}\text { Al-Mashari } \\
\text { and Zairi }\end{array}$ & 1999 & success & explicit \\
\hline 462 & $\begin{array}{l}\text { Integrating BPR with other } \\
\text { improvement approaches }\end{array}$ & 4 & 19 & 4 & 19 & 4 & 19 & $\mathrm{BPR}$ & $\begin{array}{l}\text { Al-Mashari } \\
\text { and Zairi }\end{array}$ & 1999 & success & explicit \\
\hline 463 & $\begin{array}{l}\text { Adequate identification of } \\
\text { BPR values }\end{array}$ & 4 & 19 & 4 & 19 & 5 & 25 & $\mathrm{BPR}$ & $\begin{array}{l}\text { Al-Mashari } \\
\text { and Zairi }\end{array}$ & 1999 & success & explicit \\
\hline 464 & $\begin{array}{l}\text { The effective re-engineering } \\
\text { of legacy IS }\end{array}$ & 4 & 16 & 4 & 16 & 7 & 29 & $\mathrm{BPR}$ & $\begin{array}{l}\text { Al-Mashari } \\
\text { and Zairi }\end{array}$ & 1999 & success & explicit \\
\hline 465 & Ineffective BPR teams & 4 & 24 & 4 & 24 & 4 & 24 & BPR & $\begin{array}{l}\text { Al-Mashari } \\
\text { and Zairi }\end{array}$ & 1999 & failure & explicit \\
\hline 466 & $\begin{array}{l}\text { Problems related to the inte- } \\
\text { gration mechanism, job defini- } \\
\text { tion, and allocation of respon- } \\
\text { sibilities }\end{array}$ & 4 & 24 & 4 & 24 & 6 & 28 & $\mathrm{BPR}$ & $\begin{array}{l}\text { Al-Mashari } \\
\text { and Zairi }\end{array}$ & 1999 & failure & explicit \\
\hline 467 & $\begin{array}{l}\text { Problems related to planning } \\
\text { and project management }\end{array}$ & 4 & 23 & 4 & 23 & 4 & 23 & BPR & $\begin{array}{l}\text { Al-Mashari } \\
\text { and Zairi }\end{array}$ & 1999 & failure & explicit \\
\hline 468 & Ineffective process redesign & 4 & 19 & 4 & 19 & 4 & 20 & $\mathrm{BPR}$ & $\begin{array}{l}\text { Al-Mashari } \\
\text { and Zairi }\end{array}$ & 1999 & failure & explicit \\
\hline 469 & Unrealistic expectations & 4 & 23 & 5 & 25 & 3 & 9 & BPR & $\begin{array}{l}\text { Al-Mashari } \\
\text { and Zairi }\end{array}$ & 1999 & failure & explicit \\
\hline 470 & $\begin{array}{l}\text { Ineffective re-engineering of } \\
\text { legacy IS }\end{array}$ & 4 & 16 & 4 & 16 & 7 & 29 & BPR & $\begin{array}{l}\text { Al-Mashari } \\
\text { and Zairi }\end{array}$ & 1999 & failure & explicit \\
\hline 471 & Effective communication & 3 & 10 & 3 & 10 & 3 & 10 & $\mathrm{BPR}$ & $\begin{array}{l}\text { Al-Mashari } \\
\text { and Zairi }\end{array}$ & 1999 & success & explicit \\
\hline 472 & $\begin{array}{l}\text { Empowerment of both individ- } \\
\text { uals and teams }\end{array}$ & 3 & 14 & 3 & 14 & 3 & 14 & $\mathrm{BPR}$ & $\begin{array}{l}\text { Al-Mashari } \\
\text { and Zairi }\end{array}$ & 1999 & success & explicit \\
\hline 473 & Human involvement & 3 & 9 & 3 & 9 & 3 & 9 & BPR & $\begin{array}{l}\text { Al-Mashari } \\
\text { and Zairi }\end{array}$ & 1999 & success & explicit \\
\hline 474 & Training and education & 3 & 11 & 3 & 11 & 3 & 11 & BPR & $\begin{array}{l}\text { Al-Mashari } \\
\text { and Zairi }\end{array}$ & 1999 & success & explicit \\
\hline 475 & $\begin{array}{l}\text { top management support and } \\
\text { commitment }\end{array}$ & 3 & 15 & 3 & 15 & 3 & 15 & BPR & $\begin{array}{l}\text { Al-Mashari } \\
\text { and Zairi }\end{array}$ & 1999 & success & explicit \\
\hline 476 & championship and sponsorship & 3 & 15 & 3 & 15 & 4 & 24 & $\mathrm{BPR}$ & $\begin{array}{l}\text { Al-Mashari } \\
\text { and Zairi }\end{array}$ & 1999 & success & explicit \\
\hline 477 & Adequate resources & 3 & 13 & 3 & 13 & 3 & 13 & BPR & $\begin{array}{l}\text { Al-Mashari } \\
\text { and Zairi }\end{array}$ & 1999 & success & explicit \\
\hline 478 & $\begin{array}{l}\text { External orientation and learn- } \\
\text { ing }\end{array}$ & 3 & 10 & 3 & 10 & 3 & 10 & BPR & $\begin{array}{l}\text { Al-Mashari } \\
\text { and Zairi }\end{array}$ & 1999 & success & explicit \\
\hline 479 & Effective use of consultants & 3 & 10 & 3 & 10 & 3 & 10 & BPR & $\begin{array}{l}\text { Al-Mashari } \\
\text { and Zairi }\end{array}$ & 1999 & success & explicit \\
\hline 480 & $\begin{array}{l}\text { Adequate IT investment and } \\
\text { sourcing decisions }\end{array}$ & 3 & 13 & 3 & 13 & 3 & 13 & $\mathrm{BPR}$ & $\begin{array}{l}\text { Al-Mashari } \\
\text { and Zairi }\end{array}$ & 1999 & success & explicit \\
\hline
\end{tabular}




\begin{tabular}{|c|c|c|c|c|c|c|c|c|c|c|c|c|}
\hline 481 & $\begin{array}{l}\text { Increasing the IT function } \\
\text { competency }\end{array}$ & 3 & 11 & 3 & 11 & 6 & 27 & BPR & $\begin{array}{l}\text { Al-Mashari } \\
\text { and Zairi }\end{array}$ & 1999 & success & explicit \\
\hline 482 & Problems in communication & 3 & 11 & 3 & 11 & 3 & 11 & $\mathrm{BPR}$ & $\begin{array}{l}\text { Al-Mashari } \\
\text { and Zairi }\end{array}$ & 1999 & failure & explicit \\
\hline 483 & Organisational resistance & 3 & 9 & 3 & 9 & 3 & 9 & $\mathrm{BPR}$ & $\begin{array}{l}\text { Al-Mashari } \\
\text { and Zairi }\end{array}$ & 1999 & failure & explicit \\
\hline 484 & Lack of training and education & 3 & 11 & 3 & 14 & 3 & 11 & $\mathrm{BPR}$ & $\begin{array}{l}\text { Al-Mashari } \\
\text { and Zairi }\end{array}$ & 1999 & failure & explicit \\
\hline 485 & $\begin{array}{l}\text { Problems related to commit- } \\
\text { ment, support, and leadership }\end{array}$ & 3 & 15 & 3 & 15 & 3 & 15 & BPR & $\begin{array}{l}\text { Al-Mashari } \\
\text { and Zairi }\end{array}$ & 1999 & failure & explicit \\
\hline 486 & $\begin{array}{l}\text { Problems related to champion- } \\
\text { ship and sponsorship }\end{array}$ & 3 & 15 & 3 & 15 & 4 & 24 & $\mathrm{BPR}$ & $\begin{array}{l}\text { Al-Mashari } \\
\text { and Zairi }\end{array}$ & 1999 & failure & explicit \\
\hline 487 & $\begin{array}{l}\begin{array}{l}\text { Problems related to BPR re- } \\
\text { sources }\end{array} \\
\end{array}$ & 3 & 13 & 3 & 13 & 3 & 13 & $\mathrm{BPR}$ & $\begin{array}{l}\text { Al-Mashari } \\
\text { and Zairi }\end{array}$ & 1999 & failure & explicit \\
\hline 488 & Ineffective use of consultants & 3 & 10 & 3 & 10 & 3 & 10 & $\mathrm{BPR}$ & $\begin{array}{l}\text { Al-Mashari } \\
\text { and Zairi }\end{array}$ & 1999 & failure & explicit \\
\hline 489 & $\begin{array}{l}\text { Problems related to IT invest- } \\
\text { ment and sourcing decisions }\end{array}$ & 3 & 13 & 3 & 13 & 3 & 13 & $\mathrm{BPR}$ & $\begin{array}{l}\text { Al-Mashari } \\
\text { and Zairi }\end{array}$ & 1999 & failure & explicit \\
\hline 490 & $\begin{array}{l}\text { Aligning BPR strategy with } \\
\text { corporate strategy }\end{array}$ & 5 & 26 & 5 & 26 & 5 & 26 & $\mathrm{BPR}$ & $\begin{array}{l}\text { Al-Mashari } \\
\text { and Zairi }\end{array}$ & 1999 & success & explicit \\
\hline 491 & Building a BPR vision & 5 & 26 & 5 & 26 & 6 & 28 & $\mathrm{BPR}$ & $\begin{array}{l}\text { Al-Mashari } \\
\text { and Zairi }\end{array}$ & 1999 & success & explicit \\
\hline 492 & $\begin{array}{l}\text { Adequate alignment of IT in- } \\
\text { frastructure and BPR strategy }\end{array}$ & 5 & 26 & 5 & 26 & 5 & 26 & $\mathrm{BPR}$ & $\begin{array}{l}\text { Al-Mashari } \\
\text { and Zairi }\end{array}$ & 1999 & success & explicit \\
\hline 493 & $\begin{array}{l}\text { Problems related to goals and } \\
\text { measures }\end{array}$ & 5 & 25 & 5 & 25 & 5 & 25 & $\mathrm{BPR}$ & $\begin{array}{l}\text { Al-Mashari } \\
\text { and Zairi }\end{array}$ & 1999 & failure & explicit \\
\hline 494 & $\begin{array}{l}\text { Inadequate focus and objec- } \\
\text { tives }\end{array}$ & 5 & 25 & 4 & 19 & 5 & 25 & $\mathrm{BPR}$ & $\begin{array}{l}\text { Al-Mashari } \\
\text { and Zairi }\end{array}$ & 1999 & failure & explicit \\
\hline 495 & $\begin{array}{l}\text { An adequate job integration } \\
\text { approach }\end{array}$ & 6 & 28 & 6 & 28 & 6 & 28 & $\mathrm{BPR}$ & $\begin{array}{l}\text { Al-Mashari } \\
\text { and Zairi }\end{array}$ & 1999 & success & explicit \\
\hline 496 & $\begin{array}{l}\text { Appropriate job definitions } \\
\text { and allocation of responsibili- } \\
\text { ties }\end{array}$ & 6 & 28 & 6 & 28 & 6 & 28 & $\mathrm{BPR}$ & $\begin{array}{l}\text { Al-Mashari } \\
\text { and Zairi }\end{array}$ & 1999 & success & explicit \\
\hline 497 & $\begin{array}{l}\text { Building an effective IT infra- } \\
\text { structure }\end{array}$ & 6 & 27 & 6 & 27 & 6 & 27 & $\mathrm{BPR}$ & $\begin{array}{l}\text { Al-Mashari } \\
\text { and Zairi }\end{array}$ & 1999 & success & explicit \\
\hline 498 & $\begin{array}{l}\text { Adequate measurement of IT } \\
\text { infrastructure effectiveness on } \\
\text { BPR }\end{array}$ & 6 & 27 & 6 & 27 & 6 & 27 & $\mathrm{BPR}$ & $\begin{array}{l}\text { Al-Mashari } \\
\text { and Zairi }\end{array}$ & 1999 & success & explicit \\
\hline 499 & Proper IS integration & 6 & 27 & 6 & 27 & 6 & 27 & BPR & $\begin{array}{l}\text { Al-Mashari } \\
\text { and Zairi }\end{array}$ & 1999 & success & explicit \\
\hline 500 & Improper IS integration & 6 & 27 & 6 & 27 & 6 & 27 & $\mathrm{BPR}$ & $\begin{array}{l}\text { Al-Mashari } \\
\text { and Zairi }\end{array}$ & 1999 & failure & explicit \\
\hline 501 & Effective use of software tools & 7 & 33 & 7 & 33 & 6 & 27 & $\mathrm{BPR}$ & $\begin{array}{l}\text { Al-Mashari } \\
\text { and Zairi }\end{array}$ & 1999 & success & explicit \\
\hline 502 & Inadequate IS development & 7 & 30 & 7 & 30 & 6 & 27 & BPR & $\begin{array}{l}\text { Al-Mashari } \\
\text { and Zairi }\end{array}$ & 1999 & failure & explicit \\
\hline 503 & $\begin{array}{l}\text { Risk analysis / portfolio bal- } \\
\text { ance }\end{array}$ & 1 & 5 & 1 & 5 & 1 & 5 & PPM & $\begin{array}{l}\text { Frey } \\
\text { and Buxmann }\end{array}$ & 2012 & success & explicit \\
\hline 504 & $\begin{array}{l}\text { Consideration of project inter- } \\
\text { dependencies }\end{array}$ & 4 & 23 & 4 & 23 & 4 & 23 & PPM & \begin{tabular}{|l} 
Frey \\
and Buxmann
\end{tabular} & 2012 & success & explicit \\
\hline 505 & $\begin{array}{l}\text { Portfolio segmented by asset } \\
\text { classes }\end{array}$ & 4 & 23 & 4 & 23 & 4 & 23 & PPM & $\begin{array}{l}\text { Frey } \\
\text { and Buxmann }\end{array}$ & 2012 & success & explicit \\
\hline 506 & $\begin{array}{l}\text { Measurement of costs and } \\
\text { benefits }\end{array}$ & 4 & 22 & 4 & 22 & 4 & 22 & PPM & $\begin{array}{l}\text { Frey } \\
\text { and Buxmann }\end{array}$ & 2012 & success & explicit \\
\hline 507 & $\begin{array}{l}\text { Consideration of multiple con- } \\
\text { straints (budget capacity, staff } \\
\text { capabilities, etc.) }\end{array}$ & 4 & 23 & 4 & 17 & 4 & 23 & PPM & $\begin{array}{l}\text { Frey } \\
\text { and Buxmann }\end{array}$ & 2012 & success & explicit \\
\hline
\end{tabular}




\begin{tabular}{|c|c|c|c|c|c|c|c|c|c|c|c|c|}
\hline 508 & Financial analysis & 3 & 13 & 4 & 23 & 3 & 13 & PPM & $\begin{array}{l}\text { Frey } \\
\text { and Buxmann }\end{array}$ & 2012 & success & explicit \\
\hline 509 & Top-leadership commitment & 3 & 15 & 3 & 15 & 3 & 15 & PPM & $\begin{array}{l}\text { Frey } \\
\text { and Buxmann }\end{array}$ & 2012 & success & explicit \\
\hline 510 & $\begin{array}{l}\text { Strategic fit / Strategic align- } \\
\text { ment }\end{array}$ & 5 & 26 & 5 & 26 & 5 & 26 & PPM & $\begin{array}{l}\text { Frey } \\
\text { and Buxmann }\end{array}$ & 2012 & success & explicit \\
\hline 511 & Centralized view & 5 & 26 & 5 & 26 & 6 & 28 & PPM & $\begin{array}{l}\text { Frey } \\
\text { and Buxmann }\end{array}$ & 2012 & success & explicit \\
\hline 512 & Accountability for results & 5 & 25 & 4 & 23 & 6 & 28 & PPM & $\begin{array}{l}\text { Frey } \\
\text { and Buxmann }\end{array}$ & 2012 & success & explicit \\
\hline 513 & $\begin{array}{l}\text { Experiment with or explore } \\
\text { new technology }\end{array}$ & 1 & 2 & 1 & 2 & 1 & 2 & PM & Avital & 2003 & success & explicit \\
\hline 514 & $\begin{array}{l}\text { Creative, innovative, visionary } \\
\text { thinking is encouraged }\end{array}$ & 1 & 2 & 1 & 2 & 1 & 2 & PM & Avital & 2003 & success & explicit \\
\hline 515 & Managing scope and priorities & 4 & 17 & 4 & 17 & 4 & 23 & $\mathrm{PM}$ & Avital & 2003 & success & explicit \\
\hline 516 & Dedicated hard-working staff & 4 & 24 & 4 & 24 & 4 & 24 & PM & vital & 2003 & success & explicit \\
\hline 517 & $\begin{array}{l}\text { People getting along with like- } \\
\text { able coworkers }\end{array}$ & 4 & 24 & 4 & 24 & 3 & 12 & PM & Avital & 2003 & success & explicit \\
\hline 518 & $\begin{array}{l}\text { Focus on objectives to get job } \\
\text { done }\end{array}$ & 4 & 16 & 4 & 16 & 4 & 16 & PM & Avital & 2003 & success & explicit \\
\hline 519 & $\begin{array}{l}\text { Being able to make a differ- } \\
\text { ence }\end{array}$ & 4 & 16 & 4 & 16 & 4 & 16 & PM & Avital & 2003 & success & explicit \\
\hline 520 & $\begin{array}{l}\text { Overcoming a challenge, solv- } \\
\text { ing puzzles, solving problems }\end{array}$ & 4 & 16 & 4 & 16 & 4 & 16 & PM & Avital & 2003 & success & explicit \\
\hline 521 & $\begin{array}{l}\text { Diversity of roles and respon- } \\
\text { sibilities, doing something } \\
\text { "new" }\end{array}$ & 4 & 24 & 4 & 24 & 6 & 28 & PM & Avital & 2003 & success & explicit \\
\hline 522 & $\begin{array}{l}\text { Structured process/competent } \\
\text { planning }\end{array}$ & 2 & 7 & 4 & 23 & 4 & 23 & PM & Avital & 2003 & success & explicit \\
\hline 523 & $\begin{array}{l}\text { Knowledge of the business } \\
\text { process affected by the project }\end{array}$ & 2 & 7 & 2 & 7 & 2 & 7 & PM & Avital & 2003 & success & explicit \\
\hline 524 & $\begin{array}{l}\text { Being recognized as valuable } \\
\text { by users }\end{array}$ & 2 & 6 & 3 & 8 & 2 & 6 & PM & Avital & 2003 & success & explicit \\
\hline 525 & $\begin{array}{l}\text { Top management sponsorship } \\
\text { and commitment }\end{array}$ & 3 & 15 & 3 & 15 & 3 & 15 & $\mathrm{PM}$ & Avital & 2003 & success & explicit \\
\hline 526 & Top management involvement & 3 & 15 & 3 & 15 & 3 & 15 & PM & Avital & 2003 & success & explicit \\
\hline 527 & Funds availability & 3 & 13 & 3 & 13 & 3 & 13 & PM & Avital & 2003 & success & explicit \\
\hline 528 & $\begin{array}{l}\text { Ongoing communication be- } \\
\text { tween IT and users }\end{array}$ & 3 & 11 & 3 & 11 & 3 & 10 & $\mathrm{PM}$ & Avital & 2003 & success & explicit \\
\hline 529 & $\begin{array}{l}\text { Ongoing users' involvement } \\
\text { and IT ownership }\end{array}$ & 3 & 8 & 3 & 8 & 3 & 8 & PM & Avital & 2003 & success & explicit \\
\hline 530 & Working together as a Team & 3 & 11 & 1 & 24 & 3 & 14 & PM & Avital & 2003 & success & explicit \\
\hline 531 & $\begin{array}{l}\text { Ongoing communication } \\
\text { among and within IT teams }\end{array}$ & 3 & 11 & 3 & 11 & 3 & 11 & PM & Avital & 2003 & success & explicit \\
\hline 532 & $\begin{array}{l}\text { Spirit of mutual support, shar- } \\
\text { ing, and collaboration }\end{array}$ & 3 & 11 & 3 & 14 & 3 & 11 & $\mathrm{PM}$ & Avital & 2003 & success & explicit \\
\hline 533 & $\begin{array}{l}\text { Users' buy in, organizational- } \\
\text { wide commitment }\end{array}$ & 3 & 8 & 3 & 8 & 3 & 8 & PM & Avital & 2003 & success & explicit \\
\hline 534 & Trust among stakeholders & 3 & 10 & 3 & 10 & 3 & 8 & $\mathrm{PM}$ & Avital & 2003 & success & explicit \\
\hline 535 & Personal growth potential & 3 & 11 & 3 & 11 & 3 & 9 & PM & Avital & 2003 & success & explicit \\
\hline 536 & $\begin{array}{l}\text { Career development opportu- } \\
\text { nities }\end{array}$ & 3 & 11 & 3 & 11 & 6 & 28 & PM & Avital & 2003 & success & explicit \\
\hline 537 & Learning new things & 3 & 11 & 3 & 11 & 3 & 11 & $\mathrm{PM}$ & Avital & 2003 & success & explicit \\
\hline 538 & $\begin{array}{l}\text { Having a sense of satisfaction } \\
\text { and achievement }\end{array}$ & 3 & 14 & 3 & 14 & 3 & 9 & PM & Avital & 2003 & success & explicit \\
\hline
\end{tabular}




\begin{tabular}{|c|c|c|c|c|c|c|c|c|c|c|c|c|}
\hline 539 & $\begin{array}{l}\text { Being recognized as valuable } \\
\text { for and needed to the company }\end{array}$ & 3 & 14 & 6 & 28 & 6 & 28 & PM & Avital & 2003 & success & explicit \\
\hline 540 & $\begin{array}{l}\text { Ongoing training and profes- } \\
\text { sional skills development }\end{array}$ & 3 & 11 & 3 & 11 & 3 & 11 & PM & Avital & 2003 & success & explicit \\
\hline 541 & $\begin{array}{l}\text { Learning of any kind is en- } \\
\text { couraged and supported }\end{array}$ & 3 & 11 & 1 & 2 & 3 & 11 & PM & Avital & 2003 & success & explicit \\
\hline 542 & $\begin{array}{l}\text { Focus on business objectives } \\
\text { (as opposed to technical objec- } \\
\text { tives) }\end{array}$ & 5 & 25 & 4 & 17 & 5 & 25 & PM & Avital & 2003 & success & explicit \\
\hline 543 & $\begin{array}{l}\text { Clear objectives and strategic } \\
\text { goals }\end{array}$ & 5 & 25 & 5 & 25 & 5 & 25 & PM & Avital & 2003 & success & explicit \\
\hline 544 & $\begin{array}{l}\text { Task has a clear and explicit } \\
\text { contribution or impact on the } \\
\text { business }\end{array}$ & 5 & 25 & 5 & 25 & 5 & 25 & PM & Avital & 2003 & success & explicit \\
\hline 545 & $\begin{array}{l}\text { Well-defined and bounded } \\
\text { task having clear deliverables }\end{array}$ & 5 & 25 & 5 & 25 & 4 & 23 & PM & Avital & 2003 & success & explicit \\
\hline 546 & $\begin{array}{l}\text { Sense of partnership between } \\
\text { IT and business }\end{array}$ & 5 & 26 & 5 & 26 & 3 & 8 & PM & Avital & 2003 & success & explicit \\
\hline 547 & $\begin{array}{l}\text { Infrastructure in place to sup- } \\
\text { port the developers }\end{array}$ & 6 & 27 & 6 & 27 & 6 & 27 & PM & Avital & 2003 & success & explicit \\
\hline 548 & $\begin{array}{l}\text { Proper space conducive to } \\
\text { work }\end{array}$ & 6 & 28 & 6 & 28 & 6 & 28 & PM & Avital & 2003 & success & explicit \\
\hline 549 & $\begin{array}{l}\text { Freedom to question, chal- } \\
\text { lenge or disagree }\end{array}$ & 6 & 28 & 6 & 28 & 6 & 28 & PM & Avital & 2003 & success & explicit \\
\hline 550 & $\begin{array}{l}\text { having the ability and oppor- } \\
\text { tunity to make a difference in } \\
\text { the organization }\end{array}$ & 6 & 28 & 6 & 28 & 6 & 28 & PM & Avital & 2003 & success & explicit \\
\hline 551 & $\begin{array}{l}\text { Stimulating environment, con- } \\
\text { tinuous stimulation }\end{array}$ & 6 & 28 & 6 & 28 & 6 & 28 & PM & Avital & 2003 & success & explicit \\
\hline 552 & $\begin{array}{l}\text { Knowledge of the technology } \\
\text { in use }\end{array}$ & 7 & 33 & 7 & 33 & 7 & 32 & PM & Avital & 2003 & success & explicit \\
\hline 553 & manage risk & 1 & 5 & 1 & 5 & 1 & 5 & SPM & Malinova et al. & 2014 & success & implicit \\
\hline 554 & adapt to external changes & 1 & 3 & 1 & 3 & 1 & 3 & BPM & Malinova et al. & 2014 & success & implicit \\
\hline 555 & introduce new products & 1 & 2 & 1 & 2 & 1 & 2 & BPM & Malinova et al. & 2014 & success & implicit \\
\hline 556 & optimize processes & 4 & 19 & 4 & 19 & 4 & 19 & BPM & Malinova et al. & 2014 & success & implicit \\
\hline 557 & reduce costs & 4 & 19 & 4 & 19 & 4 & 21 & BPM & Malinova et al. & 2014 & success & implicit \\
\hline 558 & reduce time & 4 & 19 & 4 & 19 & 4 & 21 & $\mathrm{BPM}$ & Malinova et al. & 2014 & success & implicit \\
\hline 559 & $\begin{array}{l}\text { assure continious [sic!] im- } \\
\text { provement }\end{array}$ & 4 & 19 & 4 & 19 & 4 & 19 & BPM & Malinova et al. & 2014 & success & implicit \\
\hline 560 & raise productivity & 4 & 16 & 4 & 16 & 4 & 16 & $\mathrm{BPM}$ & Malinova et al. & 2014 & success & implicit \\
\hline 561 & consolidate process inputs & 4 & 19 & 2 & 7 & 4 & 21 & BPM & Malinova et al. & 2014 & success & implicit \\
\hline 562 & measure & 4 & 21 & 4 & 21 & 4 & 22 & BPM & Malinova et al. & 2014 & success & implicit \\
\hline 563 & $\begin{array}{l}\text { identify and understand weak- } \\
\text { nesses of your processes }\end{array}$ & 2 & 7 & 4 & 19 & 4 & 19 & BPM & Malinova et al. & 2014 & success & implicit \\
\hline 564 & increase satisfaction & 2 & 6 & 2 & 6 & 3 & 8 & BPM & Malinova et al. & 2014 & success & implicit \\
\hline 565 & increase quality & 2 & 6 & 2 & 6 & 4 & 21 & BPM & Malinova et al. & 2014 & success & implicit \\
\hline 566 & increase process awareness & 2 & 7 & 2 & 7 & 2 & 7 & $\mathrm{BPM}$ & Malinova et al. & 2014 & success & implicit \\
\hline 567 & standardize & 2 & 7 & 2 & 7 & 2 & 7 & BPM & Malinova et al. & 2014 & success & implicit \\
\hline 568 & comply to standards & 2 & 7 & 2 & 7 & 2 & 7 & $\mathrm{BPM}$ & Malinova et al. & 2014 & success & implicit \\
\hline 569 & identify new processes & 2 & 7 & 2 & 7 & 2 & 7 & $\mathrm{BPM}$ & Malinova et al. & 2014 & success & implicit \\
\hline 570 & $\begin{array}{l}\text { faciliate employee communi- } \\
\text { cation }\end{array}$ & 3 & 11 & 3 & 11 & 3 & 11 & BPM & Malinova et al. & 2014 & success & implicit \\
\hline 571 & increase BPM knowledge & 3 & 11 & 4 & 19 & 4 & 16 & BPM & Malinova et al. & 2014 & success & implicit \\
\hline 572 & increase transparency & 3 & 11 & 3 & 11 & 3 & 11 & BPM & Malinova et al. & 2014 & success & implicit \\
\hline
\end{tabular}




\begin{tabular}{|c|c|c|c|c|c|c|c|c|c|c|c|c|}
\hline 573 & chieve proactivity & 3 & 8 & 3 & 8 & 3 & 8 & BPM & Malinova et al. & 2014 & success & implici \\
\hline 574 & ipport information system & 6 & 7 & & 27 & 6 & 27 & $\mathrm{PM}$ & Ialinova et al. & 2014 & uccess & nplici \\
\hline 575 & $\begin{array}{l}\text { rganizational change man- } \\
\text { gement }\end{array}$ & 1 & 2 & 1 & 2 & 1 & 2 & BPR & Umble et al. & 2003 & success & xplicit \\
\hline 576 & xcellent project management & 4 & 17 & 4 & 17 & 4 & 17 & BPR & Imble et al. & 2003 & success & explicit \\
\hline 577 & great implementation team & 4 & 24 & 4 & 24 & 4 & 24 & PR & nble et al. & 2003 & Iccess & plicit \\
\hline 578 & measures & 4 & 21 & 4 & 21 & 4 & 21 & BPR & Umble et al. & 2003 & uccess & xplicit \\
\hline 579 & 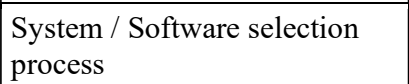 & 4 & 23 & 4 & 23 & 4 & 23 & BPR & Umble et al. & 2003 & uccess & xplicit \\
\hline 580 & & 4 & 23 & 4 & 23 & 4 & 23 & BPR & Umble et al. & 2003 & ailure & nplici \\
\hline 581 & & 3 & 15 & 3 & 15 & 3 & 15 & BPR & Umble et al. & 2003 & uccess & xplicit \\
\hline 582 & & 3 & 11 & 3 & 11 & 3 & 11 & BPR & Umble et al. & 2003 & success & explicit \\
\hline 583 & & 3 & 15 & 3 & 15 & 3 & 15 & BPR & Umble et al. & 2003 & failure & implici \\
\hline 584 & User $\mathrm{r}$ & 3 & 9 & 3 & 9 & 2 & 9 & BPR & Jmblestol & 2003 & ilune & aplici \\
\hline 585 & $\begin{array}{l}\text { Clear } \\
\text { gic goa }\end{array}$ & 5 & 25 & 5 & 25 & 5 & 25 & BPR & Umble et al. & 2003 & success & xplicit \\
\hline 586 & & 5 & 25 & 5 & 25 & 5 & 25 & BPR & Umble et al. & 2003 & failure & implici \\
\hline 587 & data & 6 & 27 & 6 & 27 & 6 & 27 & BPR & 1. & 2003 & ess & licit \\
\hline 588 & & 6 & 27 & 6 & 27 & 7 & 32 & BPR & Umble et al. & 2003 & failure & nplici \\
\hline 589 & sues & 1 & 29 & 1 & 29 & 7 & 29 & PR & Umble et al. & 003 & uccess & plicit \\
\hline 590 & - & 1 & 2 & 1 & 2 & 1 & 2 & $\overline{P R}$ & ant et al. & 2008 & uccess & plicit \\
\hline 591 & $\begin{array}{l}M \\
\text { lo }\end{array}$ & 1 & 5 & 1 & 5 & 1 & 5 & BPR & Abdolvant et al. & 2008 & failure & xplicit \\
\hline 592 & & 1 & 2 & 1 & 2 & 3 & 9 & BPR & Abdolvant et al. & 2008 & ailure & xplicit \\
\hline 593 & i- & 4 & 17 & 4 & 17 & 3 & 11 & BPR & Abdolvant et al. & 2008 & uccess & xplicit \\
\hline 594 & Teamwork performance & 4 & 24 & & 24 & 3 & 14 & BPR & Abdolvant et al. & 2008 & iccess & plicit \\
\hline 595 & Performance measurement & 4 & 22 & 4 & 22 & 4 & 21 & BPR & Abdolvant et al. & 2008 & uccess & xplicit \\
\hline 596 & Management performance & 4 & 17 & 4 & 17 & 4 & 17 & BPR & Abdolvant et al. & 2008 & uccess & aplici \\
\hline 597 & $\begin{array}{l}\text { Suffi } \\
\text { the B }\end{array}$ & 2 & 7 & 2 & 7 & 2 & 7 & BPR & t al. & 2008 & ess & licit \\
\hline 598 & Open communication & & 10 & & 10 & 1 & 10 & BPR & bdolvant et al. & 2008 & ess & xplicit \\
\hline 599 & 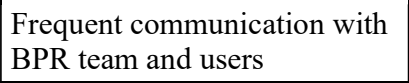 & 3 & 11 & 3 & 11 & 3 & 11 & BPR & Abdolv & 2008 & success & licit \\
\hline 600 & Employee empowerment & 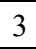 & 14 & & 14 & 2 & 9 & BPR & Abdolvant et al. & 2008 & uccess & xplicit \\
\hline 601 & Timely training and education & 3 & 11 & 3 & 11 & 2 & 11 & BPR & Abdolvant et al. & 2008 & success & explicit \\
\hline 602 & Skepticism about project result & 3 & 9 & 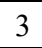 & 9 & 2 & 9 & BPR & Abdolvant et al. & 2008 & failure & explicit \\
\hline 603 & Shared vision/information & - & 25 & 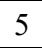 & 25 & 2 & 11 & BPR & Abdolvant et al. & 2008 & uccess & explicit \\
\hline 604 & $\begin{array}{l}\text { Realistic expectation of BPR } \\
\text { results }\end{array}$ & 5 & 25 & 5 & 25 & 3 & 9 & BPR & Abdolvant et al. & 2008 & success & xplicit \\
\hline 605 & $\begin{array}{l}\text { Confidence and trust in subor- } \\
\text { dinates }\end{array}$ & 6 & 28 & 6 & 28 & 6 & 28 & BPR & it et al. & 2008 & success & explicit \\
\hline 606 & Friendly interactions & 0 & 28 & 6 & 28 & 6 & 28 & BPR & Abdolvant et al. & 2008 & success & explicit \\
\hline 607 & Confidence and trust & 6 & 28 & 6 & 28 & 6 & 28 & BPR & Abdolvant et al. & 2008 & success & explicit \\
\hline 608 & Cooperative environment & 6 & 28 & 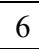 & 28 & 6 & 28 & BPR & Abdolvant et al. & 2008 & success & explicit \\
\hline 609 & Recognition among employees & 6 & 28 & 6 & 28 & 6 & 28 & BPR & Abdolvant et al. & 2008 & success & explicit \\
\hline
\end{tabular}




\begin{tabular}{|c|c|c|c|c|c|c|c|c|c|c|c|c|}
\hline 610 & Employees fear of losing job & 6 & 28 & 1 & 2 & 3 & 9 & BPR & Abdolvant et al. & 2008 & failure & explicit \\
\hline 611 & The role of IT & 7 & 33 & 7 & 33 & 6 & 27 & BPR & Abdolvant et al. & 2008 & success & explicit \\
\hline 612 & $\begin{array}{l}\text { Use of up-to-date communica- } \\
\text { tion technology }\end{array}$ & 7 & 33 & 7 & 33 & 7 & 33 & BPR & Abdolvant et al. & 2008 & success & explicit \\
\hline 613 & Adoption of IT & 7 & 33 & 7 & 33 & 7 & 33 & BPR & Abdolvant et al. & 2008 & success & explicit \\
\hline 614 & Effective Project Management & 4 & 17 & 4 & 17 & 4 & 22 & BPR & $\begin{array}{l}\text { Dezdar } \\
\text { and Ainin }\end{array}$ & 2011 & success & explicit \\
\hline 615 & $\begin{array}{l}\text { assign considerable time prior } \\
\text { to starting implementation to } \\
\text { prepare a project plan }\end{array}$ & 4 & 23 & 4 & 23 & 4 & 23 & BPR & $\begin{array}{l}\text { Dezdar } \\
\text { and Ainin }\end{array}$ & 2011 & success & implicit \\
\hline 616 & $\begin{array}{l}\text { establish the ERP implementa- } \\
\text { tion project scope and control } \\
\text { it }\end{array}$ & 4 & 22 & 4 & 22 & 4 & 22 & BPR & $\begin{array}{l}\text { Dezdar } \\
\text { and Ainin }\end{array}$ & 2011 & success & implicit \\
\hline 617 & $\begin{array}{l}\text { establish the project team and } \\
\text { their responsibilities with a } \\
\text { clear statement of work and } \\
\text { define the performance objec- } \\
\text { tives }\end{array}$ & 4 & 23 & 4 & 23 & 4 & 23 & BPR & $\begin{array}{l}\text { Dezdar } \\
\text { and Ainin }\end{array}$ & 2011 & success & implicit \\
\hline 618 & $\begin{array}{l}\text { Team Composition and Com- } \\
\text { petence }\end{array}$ & 4 & 24 & 4 & 24 & 4 & 24 & BPR & $\begin{array}{l}\text { Dezdar } \\
\text { and Ainin }\end{array}$ & 2011 & success & explicit \\
\hline 619 & $\begin{array}{l}\text { assign an experienced and rep- } \\
\text { utable project champion/man- } \\
\text { ager to lead the implementa- } \\
\text { tion }\end{array}$ & 4 & 17 & 4 & 17 & 4 & 24 & BPR & $\begin{array}{l}\text { Dezdar } \\
\text { and Ainin }\end{array}$ & 2011 & success & implicit \\
\hline 620 & $\begin{array}{l}\text { business skills of the project } \\
\text { team }\end{array}$ & 4 & 16 & 4 & 16 & 4 & 16 & BPR & $\begin{array}{l}\text { Dezdar } \\
\text { and Ainin }\end{array}$ & 2011 & success & implicit \\
\hline 621 & $\begin{array}{l}\text { requires the cooperation and } \\
\text { effort of end-users, business } \\
\text { professionals and technical ex- } \\
\text { perts }\end{array}$ & 3 & 12 & 3 & 12 & 3 & 12 & BPR & $\begin{array}{l}\text { Dezdar } \\
\text { and Ainin }\end{array}$ & 2011 & success & implicit \\
\hline 622 & $\begin{array}{l}\text { establish a team consisting of } \\
\text { all stakeholders }\end{array}$ & 3 & 12 & 4 & 24 & 4 & 24 & BPR & $\begin{array}{l}\text { Dezdar } \\
\text { and Ainin }\end{array}$ & 2011 & success & implicit \\
\hline 623 & $\begin{array}{l}\text { set up a detailed project plan } \\
\text { with clear objectives, delivera- } \\
\text { bles, realistic project mile- } \\
\text { stones and end-dates and en- } \\
\text { force them with measurable } \\
\text { results } \\
\end{array}$ & 5 & 25 & 4 & 23 & 4 & 23 & BPR & $\begin{array}{l}\text { Dezdar } \\
\text { and Ainin }\end{array}$ & 2011 & success & implicit \\
\hline 624 & $\begin{array}{l}\text { technical skills of the project } \\
\text { team }\end{array}$ & 7 & 29 & 7 & 29 & 7 & 29 & BPR & $\begin{array}{l}\text { Dezdar } \\
\text { and Ainin }\end{array}$ & 2011 & success & implicit \\
\hline 625 & $\begin{array}{l}\text { Ensure that projects can be } \\
\text { completed within four to six } \\
\text { months }\end{array}$ & 4 & 23 & 4 & 23 & 4 & 23 & BPI & Antony et al. & 2012 & success & implicit \\
\hline 626 & $\begin{array}{l}\text { Ensure that a tollgate review } \\
\text { must be performed at every } \\
\text { stage of the Six Sigma meth- } \\
\text { odology by the LSS deploy- } \\
\text { ment champion for ensuring a } \\
\text { smooth running of the projects }\end{array}$ & 4 & 22 & 4 & 22 & 4 & 23 & BPI & Antony et al. & 2012 & success & implicit \\
\hline 627 & $\begin{array}{l}\text { Select those projects which } \\
\text { have the ability to show meas- } \\
\text { urable improvements in the } \\
\text { delivery of quality associated } \\
\text { with education, operational } \\
\text { costs and timeliness parame- } \\
\text { ters }\end{array}$ & 4 & 17 & 4 & 23 & 4 & 23 & BPI & Antony et al. & 2012 & success & implicit \\
\hline 628 & $\begin{array}{l}\text { Uncompromising top manage- } \\
\text { ment support and commitment }\end{array}$ & 3 & 15 & 3 & 15 & 3 & 15 & BPI & Antony et al. & 2012 & success & explicit \\
\hline
\end{tabular}




\begin{tabular}{|c|c|c|c|c|c|c|c|c|c|c|c|c|}
\hline 629 & $\begin{array}{l}\text { Effective communication at all } \\
\text { levels vertically and horizon- } \\
\text { tally }\end{array}$ & 3 & 11 & 3 & 11 & 3 & 11 & BPI & Antony et al. & 2012 & success & explicit \\
\hline 630 & $\begin{array}{l}\text { commitment of both financial } \\
\text { and personnel resources for } \\
\text { the initiative }\end{array}$ & 3 & 13 & 5 & 26 & 5 & 25 & BPI & Antony et al. & 2012 & success & implicit \\
\hline 631 & $\begin{array}{l}\text { development of a communica- } \\
\text { tion plan }\end{array}$ & 3 & 11 & 3 & 11 & 3 & 11 & BPI & Antony et al. & 2012 & success & implicit \\
\hline 632 & reward and recognition system & 3 & 14 & 3 & 14 & 3 & 14 & BPI & Antony et al. & 2012 & success & implicit \\
\hline 633 & $\begin{array}{l}\text { build human capital by provid- } \\
\text { ing education and training to } \\
\text { employees }\end{array}$ & 3 & 11 & 3 & 11 & 3 & 11 & BPI & Antony et al. & 2012 & success & implicit \\
\hline 634 & $\begin{array}{l}\text { Projects must be aligned with } \\
\text { critical business and customer } \\
\text { issues }\end{array}$ & 3 & 8 & 3 & 8 & 3 & 8 & BPI & Antony et al. & 2012 & success & implicit \\
\hline 635 & $\begin{array}{l}\text { organisational culture is all } \\
\text { about changing the way we } \\
\text { take care of our customers } \\
\text { providing them with a world- } \\
\text { class experience }\end{array}$ & 3 & 8 & 6 & 28 & 2 & 6 & BPI & Antony et al. & 2012 & success & implicit \\
\hline 636 & $\begin{array}{l}\text { a clear strategic deployment } \\
\text { plan showing the tangible ob- } \\
\text { jectives and goals of the initia- } \\
\text { tive }\end{array}$ & 5 & 25 & 5 & 25 & 5 & 25 & BPI & Antony et al. & 2012 & success & implicit \\
\hline 637 & $\begin{array}{l}\text { clear direction and guidance } \\
\text { on deploying Six Sigma }\end{array}$ & 5 & 25 & 5 & 25 & 5 & 25 & BPI & Antony et al. & 2012 & success & implicit \\
\hline 638 & $\begin{array}{l}\text { Project objectives must be } \\
\text { clear to everyone involved in } \\
\text { the project }\end{array}$ & 5 & 25 & 5 & 25 & 5 & 25 & BPI & Antony et al. & 2012 & success & implicit \\
\hline 639 & $\begin{array}{l}\text { Developing organisational } \\
\text { readiness }\end{array}$ & 6 & 28 & 6 & 28 & 6 & 28 & BPI & Antony et al. & 2012 & success & explicit \\
\hline 640 & $\begin{array}{l}\text { Projects must be feasible to } \\
\text { execute from a resource and } \\
\text { data standpoint }\end{array}$ & 6 & 27 & 6 & 27 & 4 & 23 & BPI & Antony et al. & 2012 & success & implicit \\
\hline 641 & $\begin{array}{l}\text { Following agile-oriented pro- } \\
\text { ject management process }\end{array}$ & 1 & 3 & 2 & 7 & 1 & 3 & PM & Lam et al. & 2013 & success & explicit \\
\hline 642 & $\begin{array}{l}\text { Following agile-oriented con- } \\
\text { figuration management pro- } \\
\text { cess }\end{array}$ & 1 & 1 & 2 & 7 & 1 & 3 & PM & Lam et al. & 2013 & success & explicit \\
\hline 643 & $\begin{array}{l}\text { Project nature being non-life- } \\
\text { critical }\end{array}$ & 1 & 5 & 4 & 23 & 4 & 23 & PM & Lam et al. & 2013 & success & explicit \\
\hline 644 & $\begin{array}{l}\text { Lack of agile logistical ar- } \\
\text { rangements }\end{array}$ & 1 & 4 & 1 & 4 & 1 & 4 & PM & Lam et al. & 2013 & failure & explicit \\
\hline 645 & $\begin{array}{l}\text { Team members with high } \\
\text { competence and expertise }\end{array}$ & 4 & 24 & 4 & 24 & 4 & 24 & PM & Lam et al. & 2013 & success & explicit \\
\hline 646 & $\begin{array}{l}\text { Managers knowledgeable in } \\
\text { agile process }\end{array}$ & 4 & 17 & 4 & 17 & 1 & 3 & PM & Lam et al. & 2013 & success & explicit \\
\hline 647 & $\begin{array}{l}\text { Managers who have light- } \\
\text { touch or adaptive management } \\
\text { style }\end{array}$ & 4 & 17 & 4 & 17 & 4 & 17 & PM & Lam et al. & 2013 & success & explicit \\
\hline 648 & $\begin{array}{l}\text { Coherent, self-organizing } \\
\text { teamwork }\end{array}$ & 4 & 24 & 4 & 24 & 4 & 24 & PM & Lam et al. & 2013 & success & explicit \\
\hline 649 & Rigorous refactoring activities & 4 & 19 & 4 & 19 & 2 & 7 & $\mathrm{PM}$ & Lam et al. & 2013 & success & explicit \\
\hline 650 & Regular delivery of software & 4 & 23 & 7 & 33 & 2 & 7 & $\mathrm{PM}$ & Lam et al. & 2013 & success & explicit \\
\hline 651 & $\begin{array}{l}\text { Projects with dynamic, accel- } \\
\text { erated schedule }\end{array}$ & 4 & 23 & 4 & 23 & 1 & 2 & PM & Lam et al. & 2013 & success & explicit \\
\hline 652 & Projects with small team & 4 & 24 & 4 & 24 & 4 & 23 & PM & Lam et al. & 2013 & success & explicit \\
\hline
\end{tabular}




\begin{tabular}{|c|c|c|c|c|c|c|c|c|c|c|c|c|}
\hline 653 & $\begin{array}{l}\text { Projects with up-front cost } \\
\text { evaluation done }\end{array}$ & 4 & 23 & 4 & 23 & 4 & 23 & PM & Lam et al. & 2013 & success & explicit \\
\hline 654 & $\begin{array}{l}\text { Projects with up-front risk } \\
\text { analysis done }\end{array}$ & 4 & 23 & 4 & 23 & 1 & 5 & PM & Lam et al. & 2013 & success & explicit \\
\hline 655 & Lack of necessary skill-set & 4 & 16 & 4 & 24 & 4 & 16 & PM & Lam et al. & 2013 & failure & explicit \\
\hline 656 & $\begin{array}{l}\text { Lack of project management } \\
\text { competence }\end{array}$ & 4 & 17 & 4 & 17 & 4 & 17 & PM & Lam et al. & 2013 & failure & explicit \\
\hline 657 & Lack of team work & 4 & 24 & 4 & 24 & 3 & 14 & PM & Lam et al. & 2013 & failure & explicit \\
\hline 658 & Ill-defined project scope & 4 & 23 & 4 & 23 & 4 & 23 & $\mathrm{PM}$ & Lam et al. & 2013 & failure & explicit \\
\hline 659 & Ill-defined project planning & 4 & 23 & 4 & 23 & 4 & 23 & $\mathrm{PM}$ & Lam et al. & 2013 & failure & explicit \\
\hline 660 & $\begin{array}{l}\text { Lack of agile progress track- } \\
\text { ing mechanism }\end{array}$ & 4 & 22 & 4 & 21 & 4 & 21 & $\mathrm{PM}$ & Lam et al. & 2013 & failure & explicit \\
\hline 661 & Project Management Process & 4 & 17 & 4 & 17 & 4 & 17 & $\mathrm{PM}$ & Lam et al. & 2013 & success & explicit \\
\hline 662 & Project Definition Process & 4 & 23 & 4 & 23 & 4 & 23 & PM & Lam et al. & 2013 & success & explicit \\
\hline 663 & Ill-defined customer role & 2 & 6 & 3 & 8 & 2 & 6 & $\mathrm{PM}$ & Lam et al. & 2013 & failure & explicit \\
\hline 664 & Strong executive support & 3 & 15 & 3 & 15 & 3 & 15 & PM & & 2013 & success & explicit \\
\hline 665 & $\begin{array}{l}\text { Committed sponsor or man- } \\
\text { ager }\end{array}$ & 3 & 15 & 3 & 15 & 3 & 15 & PM & Lam et al. & 2013 & success & explicit \\
\hline 666 & $\begin{array}{l}\text { Oral culture placing high } \\
\text { value on face-to-face commu- } \\
\text { nication }\end{array}$ & 3 & 11 & 3 & 11 & 3 & 11 & PM & Lam et al. & 2013 & success & explicit \\
\hline 667 & Collocation of the whole team & 3 & 14 & 4 & 24 & 4 & 24 & PM & 1. & 2013 & success & explicit \\
\hline 668 & $\begin{array}{l}\text { Team members with great mo- } \\
\text { tivation }\end{array}$ & 3 & 14 & 3 & 14 & 3 & 14 & $\mathrm{PM}$ & Lam et al. & 2013 & success & explicit \\
\hline 669 & Good customer relationship & 3 & 8 & 3 & 8 & 2 & 6 & PM & Lam et al. & 2013 & success & explicit \\
\hline 670 & $\begin{array}{l}\text { Strong communication focus } \\
\text { with daily face-to-face meet- } \\
\text { ings }\end{array}$ & 3 & 11 & 3 & 11 & 3 & 11 & PM & Lam et al. & 2013 & success & explicit \\
\hline 671 & $\begin{array}{l}\text { Strong customer commitment } \\
\text { and presence }\end{array}$ & 3 & 8 & 3 & 8 & 3 & 8 & PM & Lam et al. & 2013 & success & explicit \\
\hline 672 & Customer having full authority & 3 & 8 & 3 & 8 & 3 & 8 & $\mathrm{PM}$ & Lam et al. & 2013 & success & explicit \\
\hline 673 & $\begin{array}{l}\text { Right amount of documenta- } \\
\text { tion }\end{array}$ & 3 & 11 & 3 & 11 & 2 & 7 & PM & Lam et al. & 2013 & success & explicit \\
\hline 674 & $\begin{array}{l}\text { Appropriate technical training } \\
\text { to team }\end{array}$ & 3 & 11 & 3 & 11 & 3 & 11 & PM & Lam et al. & 2013 & success & explicit \\
\hline 675 & Lack of executive sponsorship & 3 & 13 & 3 & 13 & 3 & 15 & $\mathrm{PM}$ & Lam et al. & 2013 & failure & explicit \\
\hline 676 & $\begin{array}{l}\text { Lack of management commit- } \\
\text { ment }\end{array}$ & 3 & 15 & 3 & 15 & 3 & 15 & PM & Lam et al. & 2013 & failure & explicit \\
\hline 677 & $\begin{array}{l}\text { Resistance from groups or in- } \\
\text { dividuals }\end{array}$ & 3 & 9 & 3 & 9 & 3 & 9 & PM & Lam et al. & 2013 & failure & explicit \\
\hline 678 & Bad customer relationship & 3 & 8 & 3 & 8 & 2 & 6 & $\mathrm{PM}$ & Lam et al. & 2013 & failure & explicit \\
\hline 679 & Lack of customer presence & 3 & 8 & 3 & 8 & 3 & 8 & $\mathrm{PM}$ & Lam et al. & 2013 & failure & explicit \\
\hline 680 & Customer Involvement & 3 & 8 & 3 & 8 & 3 & 8 & PM & Lam et al. & 2013 & success & explicit \\
\hline 681 & Management Commitment & 3 & 15 & 3 & 15 & 3 & 15 & PM & Lam et al. & 2013 & success & explicit \\
\hline 682 & $\begin{array}{l}\text { Following agile-oriented re- } \\
\text { quirement management pro- } \\
\text { cess }\end{array}$ & 5 & 25 & 2 & 7 & 1 & 3 & PM & Lam et al. & 2013 & success & explicit \\
\hline 683 & $\begin{array}{l}\text { Delivering most important fea- } \\
\text { tures first }\end{array}$ & 5 & 25 & 4 & 23 & 2 & 7 & PM & Lam et al. & 2013 & success & explicit \\
\hline 684 & $\begin{array}{l}\text { Project type being of variable } \\
\text { scope with emergent require- } \\
\text { ment }\end{array}$ & 5 & 25 & 4 & 23 & 4 & 23 & PM & Lam et al. & 2013 & success & explicit \\
\hline 685 & $\begin{array}{l}\text { Ill-defined project require- } \\
\text { ments }\end{array}$ & 5 & 25 & 4 & 23 & 5 & 25 & $\mathrm{PM}$ & Lam et al. & 2013 & failure & explicit \\
\hline
\end{tabular}




\begin{tabular}{|c|c|c|c|c|c|c|c|c|c|c|c|c|}
\hline 686 & $\begin{array}{l}\text { Cooperative organizational } \\
\text { culture instead of hierarchal }\end{array}$ & 6 & 28 & 6 & 28 & 6 & 28 & PM & Lam et al. & 2013 & success & explicit \\
\hline 687 & $\begin{array}{l}\text { Organizations where agile } \\
\text { methodology is universally ac- } \\
\text { cepted }\end{array}$ & 6 & 28 & 6 & 28 & 6 & 28 & PM & Lam et al. & 2013 & success & explicit \\
\hline 688 & \begin{tabular}{|l|} 
Facility with proper agile-style \\
work environment
\end{tabular} & 6 & 28 & 6 & 28 & 6 & 28 & $\mathrm{PM}$ & Lam et al. & 2013 & success & explicit \\
\hline 689 & $\begin{array}{l}\text { Reward system appropriate for } \\
\text { agile }\end{array}$ & 6 & 28 & 6 & 28 & 3 & 14 & $\mathrm{PM}$ & Lam et al. & 2013 & success & explicit \\
\hline 690 & $\begin{array}{l}\text { Honoring regular working } \\
\text { schedule - no overtime }\end{array}$ & 6 & 28 & 3 & 14 & 3 & 9 & $\mathrm{PM}$ & Lam et al. & 2013 & success & explicit \\
\hline 691 & $\begin{array}{l}\text { Well-defined coding standards } \\
\text { up front }\end{array}$ & 6 & 27 & 6 & 27 & 2 & 7 & $\mathrm{PM}$ & Lam et al. & 2013 & success & explicit \\
\hline 692 & $\begin{array}{l}\text { Projects with no multiple inde- } \\
\text { pendent teams }\end{array}$ & 6 & 28 & 4 & 24 & 4 & 23 & PM & Lam et al. & 2013 & success & explicit \\
\hline 693 & $\begin{array}{l}\text { Organizational culture too tra- } \\
\text { ditional }\end{array}$ & 6 & 28 & 6 & 28 & 6 & 28 & $\mathrm{PM}$ & Lam et al. & 2013 & failure & explicit \\
\hline 694 & $\begin{array}{l}\text { Organizational culture too po- } \\
\text { litical }\end{array}$ & 6 & 28 & 6 & 28 & 6 & 28 & PM & Lam et al. & 2013 & failure & explicit \\
\hline 695 & Organizational size too large & 6 & 28 & 6 & 28 & 6 & 28 & PM & Lam et al. & 2013 & failure & explicit \\
\hline 696 & $\begin{array}{l}\text { Lack of complete set of cor- } \\
\text { rect agile practices }\end{array}$ & 6 & 28 & 6 & 28 & 6 & 28 & $\mathrm{PM}$ & Lam et al. & 2013 & failure & explicit \\
\hline 697 & Pursuing simple design & 7 & 32 & 7 & 32 & 2 & 7 & PM & Lam et al. & 2013 & success & explicit \\
\hline 698 & Correct integration testing & 7 & 32 & 7 & 32 & 7 & 32 & PM & Lam et al. & 2013 & success & explicit \\
\hline 699 & $\begin{array}{l}\text { Inappropriateness of technol- } \\
\text { ogy and tools }\end{array}$ & 7 & 33 & 6 & 27 & 6 & 27 & PM & Lam et al. & 2013 & failure & explicit \\
\hline 700 & $\begin{array}{l}\text { avoid the selection of tradi- } \\
\text { tional thinkers as team mem- } \\
\text { bers }\end{array}$ & 1 & 2 & 4 & 24 & 4 & 24 & BPR & Guimaraes & 1997 & success & implicit \\
\hline 701 & $\begin{array}{l}\text { be completely open about } \\
\text { what you are doing, when and } \\
\text { why }\end{array}$ & 1 & 2 & 1 & 2 & 1 & 2 & BPR & Guimaraes & 1997 & success & implicit \\
\hline 702 & Use resources effectively & 4 & 17 & 4 & 17 & 4 & 21 & BPR & Guimaraes & 1997 & success & explicit \\
\hline 703 & $\begin{array}{l}\text { Implement new processes as } \\
\text { planned and on schedule }\end{array}$ & 4 & 20 & 4 & 22 & 4 & 22 & $\mathrm{BPR}$ & Guimaraes & 1997 & success & explicit \\
\hline 704 & use of project champions & 4 & 24 & 4 & 24 & 4 & 24 & $\mathrm{BPR}$ & Guimaraes & 1997 & success & implicit \\
\hline 705 & $\begin{array}{l}\text { Utilize hands-on experience in } \\
\text { reengineering diverse pro- } \\
\text { cesses }\end{array}$ & 4 & 20 & 4 & 23 & 4 & 19 & BPR & Guimaraes & 1997 & success & explicit \\
\hline 706 & $\begin{array}{l}\text { Use concept design phase to } \\
\text { develop a rough-cut design } \\
\text { and to identify major issues }\end{array}$ & 4 & 19 & 4 & 23 & 4 & 23 & BPR & Guimaraes & 1997 & success & explicit \\
\hline 707 & $\begin{array}{l}\text { Determine all setup details, } \\
\text { tooling, scheduling, mainte- } \\
\text { nance, storage, replenishment, } \\
\text { quality, etc. before implemen- } \\
\text { tation }\end{array}$ & 4 & 23 & 4 & 23 & 4 & 23 & BPR & Guimaraes & 1997 & success & explicit \\
\hline 708 & $\begin{array}{l}\text { Simplify material flow, logis- } \\
\text { tics, planning, and other dis- } \\
\text { tinct operations by using } \\
\text { group technology }\end{array}$ & 4 & 20 & 6 & 27 & 7 & 29 & BPR & Guimaraes & & success & explicit \\
\hline 709 & \begin{tabular}{|l|} 
Use process mapping to distin- \\
guish productive activities \\
from non-value-added activi- \\
ties \\
\end{tabular} & 4 & 19 & 4 & 19 & 4 & 19 & BPR & Guimaraes & & success & explicit \\
\hline 710 & $\begin{array}{l}\text { Reduce cost and response } \\
\text { times by automation }\end{array}$ & 4 & 19 & 4 & 19 & 4 & 19 & BPR & Guimaraes & 1997 & success & explicit \\
\hline
\end{tabular}




\begin{tabular}{|c|c|c|c|c|c|c|c|c|c|c|c|c|}
\hline 711 & $\begin{array}{l}\text { Desire for continuous perfor- } \\
\text { mance improvement }\end{array}$ & 4 & 19 & 4 & 19 & 4 & 19 & BPR & Guimaraes & 1997 & success & explicit \\
\hline 712 & $\begin{array}{l}\text { Use surveys to determine } \\
\text { what's working and what's not }\end{array}$ & 4 & 19 & 4 & 19 & 3 & 11 & BPR & Guimaraes & 1997 & success & explicit \\
\hline 713 & $\begin{array}{l}\text { BPR motivated by customer } \\
\text { demands and competitive } \\
\text { pressures }\end{array}$ & 2 & 6 & 2 & 6 & 3 & 8 & BPR & Guimaraes & 1997 & success & explicit \\
\hline 714 & $\begin{array}{l}\text { Focus on the outcome rather } \\
\text { than task }\end{array}$ & 2 & 6 & 2 & 6 & 2 & 6 & BPR & Guimaraes & 1997 & success & explicit \\
\hline 715 & $\begin{array}{l}\text { Use industry specialists and } \\
\text { outside assistance }\end{array}$ & 3 & 10 & 3 & 10 & 3 & 10 & BPR & Guimaraes & 1997 & success & explicit \\
\hline 716 & $\begin{array}{l}\text { Reeducate and retrain workers } \\
\text { on what BPR actually is }\end{array}$ & 3 & 11 & 3 & 11 & 3 & 11 & BPR & Guimaraes & 1997 & success & explicit \\
\hline 717 & $\begin{array}{l}\text { improve relationships with } \\
\text { suppliers }\end{array}$ & 3 & 12 & 3 & 12 & 3 & 12 & BPR & Guimaraes & 1997 & success & implicit \\
\hline 718 & education and re-education & 3 & 11 & 3 & 11 & 3 & 11 & BPR & Guimaraes & 1997 & success & implicit \\
\hline 719 & $\begin{array}{l}\text { reconsider mechanisms for re- } \\
\text { ward and recognition }\end{array}$ & 3 & 14 & 3 & 14 & 3 & 14 & $\mathrm{BPR}$ & Guimaraes & 1997 & success & implicit \\
\hline 720 & $\begin{array}{l}\text { Share and exchange infor- } \\
\text { mation willingly }\end{array}$ & 3 & 11 & 3 & 11 & 3 & 11 & $\mathrm{BPR}$ & Guimaraes & 1997 & success & explicit \\
\hline 721 & \begin{tabular}{|l|} 
Schedule meetings between \\
project manager and each \\
level of project structure regu- \\
larly
\end{tabular} & 3 & 11 & 3 & 11 & 3 & 11 & $\mathrm{BPR}$ & Guimaraes & & success & explicit \\
\hline 722 & \begin{tabular}{|l|} 
Revise procedures that focus \\
on satisfying internal demands \\
rather than the marketplace
\end{tabular} & 3 & 8 & 2 & 7 & 3 & 8 & $\mathrm{BPR}$ & Guimaraes & 1997 & success & explicit \\
\hline 723 & $\begin{array}{l}\text { Empower workers so that do- } \\
\text { ers are decision makers }\end{array}$ & 3 & 14 & 3 & 11 & 3 & 9 & BPR & Guimaraes & 1997 & success & explicit \\
\hline 724 & $\begin{array}{l}\text { BPR initiated and led from the } \\
\text { top-down by senior-level man- } \\
\text { agement }\end{array}$ & 3 & 15 & 3 & 15 & 3 & 15 & BPR & Guimaraes & 1997 & success & explicit \\
\hline 725 & $\begin{array}{l}\text { BPR motivated by chief exec- } \\
\text { utive willing to be held ac- } \\
\text { countable for project success }\end{array}$ & 3 & 15 & 3 & 15 & 3 & 15 & BPR & Guimaraes & & success & explicit \\
\hline 726 & $\begin{array}{l}\text { Develop and communicate } \\
\text { clear written mission and vi- } \\
\text { sion statements }\end{array}$ & 5 & 25 & 5 & 25 & 5 & 25 & BPR & Guimaraes & 1997 & success & explicit \\
\hline 727 & \begin{tabular}{|l|} 
Target only a few critical \\
(though cross-functional) busi- \\
ness processes
\end{tabular} & 5 & 25 & 2 & 7 & 1 & 2 & BPR & Guimaraes & 1997 & success & explicit \\
\hline 728 & $\begin{array}{l}\text { Create an enabling charter that } \\
\text { describes the BPR program } \\
\text { and support of management }\end{array}$ & 5 & 25 & 5 & 25 & 3 & 15 & BPR & Guimaraes & & success & explicit \\
\hline 729 & $\begin{array}{l}\text { Adopt an integrated approach } \\
\text { to IT and business planning }\end{array}$ & 5 & 26 & 5 & 26 & 5 & 26 & BPR & Guimaraes & 1997 & success & explicit \\
\hline 730 & $\begin{array}{l}\text { Develop a defined project or- } \\
\text { ganization }\end{array}$ & 6 & 28 & 4 & 23 & 6 & 28 & BPR & Guimaraes & 1997 & success & explicit \\
\hline 731 & $\begin{array}{l}\text { View technology as an ena- } \\
\text { bler, not as a solution }\end{array}$ & 6 & 27 & 6 & 27 & 6 & 27 & BPR & Guimaraes & 1997 & success & explicit \\
\hline 732 & $\begin{array}{l}\text { establishment and utilization } \\
\text { of a formal experimental } \\
\text { methodology }\end{array}$ & 1 & 5 & 1 & 5 & 4 & 23 & Digit* & $\begin{array}{l}\text { Siegel } \\
\text { and Madni }\end{array}$ & 2014 & success & explicit \\
\hline 733 & $\begin{array}{l}\text { empowerment of the develop- } \\
\text { ment team to implement a } \\
\text { number of methodological in- } \\
\text { novations, in conjunction with }\end{array}$ & 1 & 2 & 5 & 26 & 3 & 9 & Digit* & $\begin{array}{l}\text { Siegel } \\
\text { and Madni }\end{array}$ & 2014 & success & explicit \\
\hline
\end{tabular}




\begin{tabular}{|c|c|c|c|c|c|c|c|c|c|c|c|c|}
\hline & $\begin{array}{l}\text { all of the technological inno- } \\
\text { vations }\end{array}$ & & & & & & & & & & & \\
\hline 734 & $\begin{array}{l}\text { willingness to push experi- } \\
\text { ments to the point of failure, to } \\
\text { uncover behavioral boundaries }\end{array}$ & 1 & 5 & 1 & 5 & 1 & 5 & Digit* & $\begin{array}{l}\text { Siegel } \\
\text { and Madni }\end{array}$ & 2014 & success & explicit \\
\hline 735 & $\begin{array}{l}\text { willingness to carry multiple } \\
\text { options in the design } \\
\text { tradespace for an unusually } \\
\text { long time to mitigate a signifi- } \\
\text { cant portion of each risk item } \\
\text { before down-selecting to a sin- } \\
\text { gle preferred approach }\end{array}$ & 4 & 23 & 4 & 23 & 1 & 5 & Digit* & $\begin{array}{l}\text { Siegel } \\
\text { and Madni }\end{array}$ & 2014 & success & explicit \\
\hline 736 & $\begin{array}{l}\text { willingness to manage the pro- } \\
\text { gram with schedule as the in- } \\
\text { dependent variable, and being } \\
\text { willing to adjust the order and } \\
\text { timing of specific capabilities } \\
\text { to keep on schedule }\end{array}$ & 4 & 22 & 1 & 3 & 5 & 25 & Digit* & $\begin{array}{l}\text { Siegel } \\
\text { and Madni }\end{array}$ & 2014 & success & explicit \\
\hline 737 & $\begin{array}{l}\text { a strong, top-down commit- } \\
\text { ment }\end{array}$ & 3 & 15 & 3 & 15 & 3 & 15 & Digit* & $\begin{array}{l}\text { Siegel } \\
\text { and Madni }\end{array}$ & 2014 & success & explicit \\
\hline 738 & $\begin{array}{l}\text { Using project plans as work- } \\
\text { ing documents }\end{array}$ & 1 & 3 & 3 & 11 & 3 & 11 & PM & Clarke & 1999 & success & explicit \\
\hline 739 & $\begin{array}{l}\text { Breaking the project into 'bite } \\
\text { sized chunks' }\end{array}$ & 4 & 23 & 4 & 23 & 4 & 23 & PM & Clarke & 1999 & success & explicit \\
\hline 740 & $\begin{array}{l}\text { Develop an auditing tool for } \\
\text { project management }\end{array}$ & 4 & 22 & 4 & 22 & 4 & 22 & PM & Clarke & 1999 & success & implicit \\
\hline 741 & $\begin{array}{l}\text { Communicate the importance } \\
\text { of the final stages in a project }\end{array}$ & 4 & 17 & 5 & 25 & 5 & 25 & PM & Clarke & 1999 & success & implicit \\
\hline 742 & $\begin{array}{l}\text { Define the 'bite sized chunks' } \\
\text { at the outset of the project (to } \\
\text { counteract the project overload } \\
\text { syndrome) }\end{array}$ & 4 & 23 & 4 & 23 & 4 & 23 & PM & Clarke & 1999 & success & implicit \\
\hline 743 & $\begin{array}{l}\text { Focus on key success factors } \\
\text { as a first stage to standardisa- } \\
\text { tion }\end{array}$ & 2 & 7 & 2 & 7 & 2 & 7 & PM & Clarke & 1999 & success & implicit \\
\hline 744 & $\begin{array}{l}\text { Communication throughout } \\
\text { the project }\end{array}$ & 3 & 11 & 3 & 11 & 3 & 11 & PM & Clarke & 1999 & success & explicit \\
\hline 745 & $\begin{array}{l}\text { Improve information flows } \\
\text { thoughout the organisation }\end{array}$ & 3 & 11 & 3 & 11 & 3 & 11 & PM & Clarke & 1999 & success & implicit \\
\hline 746 & $\begin{array}{l}\text { Build individualism through } \\
\text { effective communication }\end{array}$ & 3 & 11 & 3 & 11 & 3 & 11 & PM & Clarke & 1999 & success & implicit \\
\hline 747 & $\begin{array}{l}\text { Build confidence through bet- } \\
\text { ter communication }\end{array}$ & 3 & 11 & 3 & 11 & 3 & 11 & PM & Clarke & 1999 & success & implicit \\
\hline 748 & Clear objectives and scope & 5 & 25 & 5 & 25 & 5 & 25 & PM & Clarke & 1999 & success & explicit \\
\hline 749 & $\begin{array}{l}\text { Increase awareness of both } \\
\text { perceived and actual benefits }\end{array}$ & 5 & 25 & 5 & 25 & 5 & 25 & PM & Clarke & 1999 & success & implicit \\
\hline 750 & project management & 4 & 17 & 4 & 17 & 4 & 17 & $\mathrm{BPM}$ & \begin{tabular}{|l} 
Bai \\
and Sarkis
\end{tabular} & 2013 & success & implicit \\
\hline 751 & Project management & 4 & 17 & 4 & 17 & 4 & 17 & BPM & $\begin{array}{l}\text { Bai } \\
\text { and Sarkis }\end{array}$ & 2013 & success & explicit \\
\hline 752 & Performance measurement & 4 & 21 & 4 & 21 & 4 & 21 & BPM & $\begin{array}{l}\text { Bai } \\
\text { and Sarkis }\end{array}$ & 2013 & success & explicit \\
\hline 753 & top management support & 3 & 15 & 3 & 15 & 3 & 15 & BPM & $\begin{array}{l}\text { Bai } \\
\text { and Sarkis }\end{array}$ & 2013 & success & implicit \\
\hline 754 & communication & 3 & 11 & 3 & 11 & 3 & 11 & BPM & $\begin{array}{l}\text { Bai } \\
\text { and Sarkis }\end{array}$ & 2013 & success & implicit \\
\hline 755 & Top management support & 3 & 15 & 3 & 15 & 3 & 15 & BPM & $\begin{array}{l}\text { Bai } \\
\text { and Sarkis }\end{array}$ & 2013 & success & explicit \\
\hline
\end{tabular}




\begin{tabular}{|c|c|c|c|c|c|c|c|c|c|c|c|c|}
\hline 756 & User Focus & 3 & 8 & 3 & 8 & 3 & 8 & $\mathrm{BPM}$ & $\begin{array}{l}\text { Bai } \\
\text { and Sarkis }\end{array}$ & 2013 & success & explicit \\
\hline 757 & Stategic alignment & 5 & 26 & 5 & 26 & 5 & 26 & BPM & $\begin{array}{l}\text { Bai } \\
\text { and Sarkis }\end{array}$ & 2013 & success & explicit \\
\hline 758 & inter-departmental cooperation & 6 & 28 & 5 & 26 & 3 & 11 & $\mathrm{BPM}$ & $\begin{array}{l}\text { Bai } \\
\text { and Sarkis }\end{array}$ & 2013 & success & implicit \\
\hline 759 & Information technology & 6 & 27 & 6 & 27 & 6 & 27 & $\mathrm{BPM}$ & $\begin{array}{l}\text { Bai } \\
\text { and Sarkis }\end{array}$ & 2013 & success & explicit \\
\hline 760 & Collaborative environment & 6 & 28 & 6 & 28 & 6 & 28 & $\mathrm{BPM}$ & $\begin{array}{l}\text { Bai } \\
\text { and Sarkis }\end{array}$ & 2013 & success & explicit \\
\hline 761 & Culture & 6 & 28 & 6 & 28 & 6 & 28 & $\mathrm{BPM}$ & $\begin{array}{l}\text { Bai } \\
\text { and Sarkis }\end{array}$ & 2013 & success & explicit \\
\hline 762 & $\begin{array}{l}\text { Organizations inability to be } \\
\text { open about IS failure }\end{array}$ & 1 & 5 & 1 & 5 & 1 & 5 & PM & Hughes et al. & 2017 & failure & explicit \\
\hline 763 & $\begin{array}{l}\text { Failure is multi-dimensional } \\
\text { with interconnected factors }\end{array}$ & 1 & 5 & 1 & 5 & 1 & 5 & PM & Hughes et al. & 2017 & failure & explicit \\
\hline 764 & $\begin{array}{l}\text { Poor assessment and manage- } \\
\text { ment of risks }\end{array}$ & 1 & 5 & 1 & 5 & 1 & 5 & PM & Hughes et al. & 2017 & failure & explicit \\
\hline 765 & $\begin{array}{l}\text { Factors relating to Poor } \\
\text { Change Management and User } \\
\text { Resistance }\end{array}$ & 1 & 2 & 1 & 2 & 1 & 2 & PM & Hughes et al. & 2017 & failure & explicit \\
\hline 766 & $\begin{array}{l}\text { Inadequate Management } \\
\text { Structure }\end{array}$ & 1 & 3 & 6 & 28 & 6 & 28 & PM & Hughes et al. & 2017 & failure & explicit \\
\hline 767 & $\begin{array}{l}\text { plan for users changing their } \\
\text { minds and ensure the required } \\
\text { controls are in place }\end{array}$ & 1 & 3 & 1 & 3 & 1 & 2 & PM & Hughes et al. & 2017 & success & implicit \\
\hline 768 & $\begin{array}{l}\text { plan for users changing their } \\
\text { minds and ensure the controls } \\
\text { are in place to manage this ef- } \\
\text { fectively }\end{array}$ & 1 & 3 & 1 & 3 & 1 & 2 & PM & Hughes et al. & 2017 & success & implicit \\
\hline 769 & $\begin{array}{l}\text { Poor project management is a } \\
\text { common failure factor }\end{array}$ & 4 & 17 & 4 & 17 & 4 & 17 & PM & Hughes et al. & 2017 & failure & explicit \\
\hline 770 & Poor management skills & 4 & 17 & 4 & 17 & 4 & 17 & $\mathrm{PM}$ & Hughes et al. & 2017 & failure & explicit \\
\hline 771 & $\begin{array}{l}\text { Project too big to fail, exec } \\
\text { lacking courage to stop project }\end{array}$ & 4 & 23 & 4 & 23 & 4 & 17 & PM & Hughes et al. & 2017 & failure & explicit \\
\hline 772 & $\begin{array}{l}\text { Difficulties faced by project } \\
\text { managers on complex projects }\end{array}$ & 4 & 17 & 4 & 17 & 4 & 23 & PM & Hughes et al. & 2017 & failure & explicit \\
\hline 773 & $\begin{array}{l}\text { Large projects have a virtual } \\
\text { zero chance of being success- } \\
\text { ful }\end{array}$ & 4 & 23 & 4 & 23 & 4 & 23 & PM & Hughes et al. & 2017 & failure & explicit \\
\hline 774 & Lack of PM methodology & 4 & 23 & 4 & 23 & 4 & 23 & $\mathrm{PM}$ & Hughes et al. & 2017 & failure & explicit \\
\hline 775 & Elongated time-scales & 4 & 23 & 4 & 23 & 4 & 22 & $\mathrm{PM}$ & Hughes et al. & 2017 & failure & explicit \\
\hline 776 & $\begin{array}{l}\text { Lack of time devoted to PM } \\
\text { based tasks }\end{array}$ & 4 & 23 & 4 & 23 & 4 & 23 & PM & Hughes et al. & 2017 & failure & explicit \\
\hline 777 & $\begin{array}{l}\text { Inaccurate estimating on large } \\
\text { projects }\end{array}$ & 4 & 23 & 4 & 23 & 4 & 23 & PM & Hughes et al. & 2017 & failure & explicit \\
\hline 778 & $\begin{array}{l}\text { If organizations paid attention } \\
\text { to EWS during first } 20 \% \text { of } \\
\text { project lifecycle, probability } \\
\text { of successful outcomes is } \\
\text { greatly increased }\end{array}$ & 4 & 22 & 4 & 22 & 4 & 22 & PM & Hughes et al. & 2017 & failure & explicit \\
\hline 779 & $\begin{array}{l}\text { Project management and pro- } \\
\text { cess failings were biggest con- } \\
\text { tributors to failure }\end{array}$ & 4 & 17 & 4 & 17 & 4 & 17 & PM & Hughes et al. & 2017 & failure & explicit \\
\hline 780 & $\begin{array}{l}\text { Failure linked to inability to } \\
\text { deliver desired value }\end{array}$ & 4 & 23 & 2 & 6 & 2 & 6 & PM & Hughes et al. & 2017 & failure & explicit \\
\hline
\end{tabular}




\begin{tabular}{|c|c|c|c|c|c|c|c|c|c|c|c|c|}
\hline 781 & $\begin{array}{l}\text { Project management seems to } \\
\text { have an over reliance on task } \\
\text { based activities }\end{array}$ & 4 & 17 & 4 & 17 & 4 & 17 & PM & Hughes et al. & 2017 & failure & explicit \\
\hline 782 & Poor Project Planning & 4 & 23 & 4 & 23 & 4 & 23 & PM & Hughes et al. & 2017 & failure & explicit \\
\hline 783 & $\begin{array}{l}\text { Poor Project Management and } \\
\text { Project Planning }\end{array}$ & 4 & 17 & 4 & 17 & 4 & 23 & PM & Hughes et al. & 2017 & failure & explicit \\
\hline 784 & $\begin{array}{l}\text { Project Too Large and Com- } \\
\text { plex }\end{array}$ & 4 & 23 & 4 & 23 & 4 & 23 & PM & Hughes et al. & 2017 & failure & explicit \\
\hline 785 & Staff Turnover & 4 & 24 & 4 & 24 & 3 & 9 & $\mathrm{PM}$ & Hughes et al. & 2017 & failure & explicit \\
\hline 786 & $\begin{array}{l}\text { Poor Business Case, Objec- } \\
\text { tives and Evaluation Stage }\end{array}$ & 4 & 23 & 4 & 23 & 4 & 23 & PM & Hughes et al. & 2017 & failure & explicit \\
\hline 787 & $\begin{array}{l}\text { Inadequate Post Mortem Pro- } \\
\text { cess }\end{array}$ & 4 & 19 & 4 & 19 & 3 & 11 & PM & Hughes et al. & 2017 & failure & explicit \\
\hline 788 & managerial leadership skills & 4 & 17 & 4 & 17 & 4 & 17 & PM & Hughes et al. & 2017 & failure & explicit \\
\hline 789 & $\begin{array}{l}\text { break up the project into } \\
\text { smaller separate projects each } \\
\text { with their individual business } \\
\text { case, infrastructure and re- } \\
\text { source requirements with de- } \\
\text { fined dependencies to align } \\
\text { deliverables and key benefits }\end{array}$ & 4 & 23 & 4 & 23 & 4 & 23 & PM & Hughes et al. & 2017 & success & implicit \\
\hline 790 & $\begin{array}{l}\text { embrace the project audit pro- } \\
\text { cess and not to resist the pro- } \\
\text { cess }\end{array}$ & 4 & 22 & 4 & 22 & 4 & 22 & PM & Hughes et al. & 2017 & success & implicit \\
\hline 791 & $\begin{array}{l}\text { Tailor the method to suit the } \\
\text { project and organization }\end{array}$ & 4 & 23 & 4 & 23 & 4 & 23 & PM & Hughes et al. & 2017 & success & implicit \\
\hline 792 & $\begin{array}{l}\text { Do not use time, cost and } \\
\text { quality as the measure of as- } \\
\text { sessing the success of a project }\end{array}$ & 4 & 22 & 4 & 22 & 4 & 22 & PM & Hughes et al. & 2017 & success & implicit \\
\hline 793 & $\begin{array}{l}\text { have sufficient knowledge and } \\
\text { experience of change manage- } \\
\text { ment principles and processes } \\
\text { to ensure the project has the } \\
\text { best chance of success }\end{array}$ & 4 & 17 & 4 & 17 & 1 & 2 & PM & Hughes et al. & 2017 & success & implicit \\
\hline 794 & $\begin{array}{l}\text { Key to identify early stage } \\
\text { threats to IS project }\end{array}$ & 2 & 7 & 4 & 21 & 4 & 23 & PM & Hughes et al. & 2017 & failure & explicit \\
\hline 795 & $\begin{array}{l}\text { Increasing range of complex } \\
\text { issues affecting IS projects }\end{array}$ & 2 & 7 & 4 & 23 & 5 & 25 & PM & Hughes et al. & 2017 & failure & explicit \\
\hline 796 & $\begin{array}{l}\text { Expect users to change their } \\
\text { minds }\end{array}$ & 2 & 6 & 2 & 6 & 2 & 6 & PM & Hughes et al. & 2017 & success & implicit \\
\hline 797 & \begin{tabular}{|l|} 
Poor communication skills \\
\end{tabular} & 3 & 11 & 3 & 11 & 3 & 11 & $\mathrm{PM}$ & Hughes et al. & 2017 & failure & explicit \\
\hline 798 & $\begin{array}{l}\text { Poor project sponsorship is a } \\
\text { key factor that leads to failure }\end{array}$ & 3 & 13 & 3 & 13 & 3 & 13 & PM & Hughes et al. & 2017 & failure & explicit \\
\hline 799 & $\begin{array}{l}\text { Assertion that organization put } \\
\text { price before quality }\end{array}$ & 3 & 13 & 6 & 28 & 4 & 23 & PM & Hughes et al. & 2017 & failure & explicit \\
\hline 800 & $\begin{array}{l}\text { Incomplete and inconsistent } \\
\text { training }\end{array}$ & 3 & 11 & 3 & 11 & 3 & 11 & PM & Hughes et al. & 2017 & failure & explicit \\
\hline 801 & $\begin{array}{l}\text { Over-reliance on an external } \\
\text { contractor }\end{array}$ & 3 & 12 & 3 & 12 & 3 & 12 & PM & Hughes et al. & 2017 & failure & explicit \\
\hline 802 & impact on staff commitment & 3 & 9 & 3 & 9 & 3 & 9 & $\mathrm{PM}$ & Hughes et al. & 2017 & failure & explicit \\
\hline 803 & $\begin{array}{l}\text { No effort made to fully under- } \\
\text { stand what went wrong }\end{array}$ & 3 & 11 & 1 & 5 & 3 & 11 & PM & Hughes et al. & 2017 & failure & explicit \\
\hline 804 & $\begin{array}{l}\text { Lack of management commit- } \\
\text { ment }\end{array}$ & 3 & 15 & 3 & 15 & 3 & 15 & PM & Hughes et al. & 2017 & failure & explicit \\
\hline 805 & $\begin{array}{l}\text { No effort made to find out } \\
\text { what went wrong and learn } \\
\text { from past mistakes }\end{array}$ & 3 & 11 & 1 & 5 & 3 & 11 & PM & Hughes et al. & 2017 & failure & explicit \\
\hline
\end{tabular}




\begin{tabular}{|c|c|c|c|c|c|c|c|c|c|c|c|c|}
\hline 806 & $\begin{array}{l}\text { Lack of top management com- } \\
\text { mitment }\end{array}$ & 3 & 15 & 3 & 15 & 3 & 15 & PM & Hughes et al. & 2017 & failure & explicit \\
\hline 807 & $\begin{array}{l}\text { Stakeholder support for sys- } \\
\text { tem is critical }\end{array}$ & 3 & 8 & 3 & 8 & 7 & 33 & PM & Hughes et al. & 2017 & failure & explicit \\
\hline 808 & $\begin{array}{l}\text { Social aspects associated with } \\
\text { IS failure ignored }\end{array}$ & 3 & 14 & 1 & 5 & 3 & 9 & PM & Hughes et al. & 2017 & failure & explicit \\
\hline 809 & $\begin{array}{l}\text { Top management support is } \\
\text { the key critical factor }\end{array}$ & 3 & 15 & 3 & 15 & 3 & 15 & PM & Hughes et al. & 2017 & failure & explicit \\
\hline 810 & $\begin{array}{l}\text { Risk and Budget Management } \\
\text { Failings }\end{array}$ & 3 & 13 & 3 & 13 & 3 & 13 & $\mathrm{PM}$ & Hughes et al. & 2017 & failure & explicit \\
\hline 811 & Poor Executive Support & 3 & 15 & 3 & 15 & 3 & 9 & $\mathrm{PM}$ & Hughes et al. & 2017 & failure & explicit \\
\hline 812 & Poor Executive Sponsorship & 3 & 13 & 3 & 13 & 3 & 13 & $\mathrm{PM}$ & Hughes et al. & 2017 & failure & explicit \\
\hline 813 & $\begin{array}{l}\text { Poor Contractor and Stake- } \\
\text { holder Relationship }\end{array}$ & 3 & 8 & 3 & 12 & 3 & 8 & PM & Hughes et al. & 2017 & failure & explicit \\
\hline 814 & Staff Commitment & 3 & 9 & 3 & 9 & 3 & 9 & $\mathrm{PM}$ & Hughes et al. & 2017 & failure & explicit \\
\hline 815 & Staff Motivation & 3 & 14 & 3 & 14 & 3 & 9 & $\mathrm{PM}$ & Hughes et al. & 2017 & failure & explicit \\
\hline 816 & strategic client interaction & 3 & 8 & 3 & 8 & 3 & 8 & PM & Hughes et al. & 2017 & failure & explicit \\
\hline 817 & $\begin{array}{l}\text { motivational and positive lead- } \\
\text { ership skills }\end{array}$ & 3 & 14 & 3 & 14 & 4 & 17 & PM & Hughes et al. & 2017 & failure & explicit \\
\hline 818 & $\begin{array}{l}\text { ensure that project post mor- } \\
\text { tems form a key element of } \\
\text { the project methodology }\end{array}$ & 3 & 11 & 4 & 23 & 3 & 11 & PM & Hughes et al. & 2017 & success & implicit \\
\hline 819 & $\begin{array}{l}\text { Ensure the suitability and ex- } \\
\text { perience of the nominated } \\
\text { sponsor is assessed at an early } \\
\text { stage and work to bridge any } \\
\text { gaps quickly }\end{array}$ & 3 & 13 & 3 & 13 & 4 & 23 & PM & Hughes et al. & 2017 & success & implicit \\
\hline 820 & $\begin{array}{l}\text { the people related aspects are } \\
\text { fundamental to benefit realiza- } \\
\text { tion and cannot be ignored }\end{array}$ & 3 & 14 & 3 & 14 & 3 & 9 & $\mathrm{PM}$ & Hughes et al. & 2017 & success & implicit \\
\hline 821 & $\begin{array}{l}\text { Poor Requirements Manage- } \\
\text { ment }\end{array}$ & 5 & 25 & 5 & 25 & 5 & 25 & PM & Hughes et al. & 2017 & failure & explicit \\
\hline 822 & $\begin{array}{l}\text { organizational and political } \\
\text { complexities are the key rea- } \\
\text { sons for high failure rates in } \\
\text { public sector projects }\end{array}$ & 6 & 28 & 6 & 28 & 6 & 28 & PM & Hughes et al. & 2017 & failure & explicit \\
\hline 823 & $\begin{array}{l}\text { High rates of failure in devel- } \\
\text { oping countries due to context } \\
\text { gaps and local actuality factors }\end{array}$ & 6 & 28 & 6 & 28 & 6 & 28 & PM & Hughes et al. & 2017 & failure & explicit \\
\hline 824 & $\begin{array}{l}\text { Do not underestimate the im- } \\
\text { portance of experience and } \\
\text { pragmatic application of pro- } \\
\text { ject management to fit with } \\
\text { the needs and cultural aspects } \\
\text { of the organization }\end{array}$ & 6 & 28 & 6 & 28 & 4 & 17 & PM & Hughes et al. & 2017 & success & implicit \\
\hline 825 & $\begin{array}{l}\text { Exec bias toward more risky } \\
\text { technology option }\end{array}$ & 7 & 30 & 7 & 30 & 1 & 5 & PM & Hughes et al. & 2017 & failure & explicit \\
\hline 826 & No feasibility undertaken & 7 & 32 & 7 & 32 & 4 & 23 & PM & Hughes et al. & 2017 & failure & explicit \\
\hline 827 & $\begin{array}{l}\text { Software was incomplete and } \\
\text { not stable }\end{array}$ & 7 & 33 & 7 & 33 & 7 & 33 & $\mathrm{PM}$ & Hughes et al. & 2017 & failure & explicit \\
\hline 828 & technical leadership skills & 1 & 30 & $T$ & 17 & 7 & 30 & $\mathrm{PM}$ & Hughes et al. & 2017 & failure & explicit \\
\hline 829 & Change management & 1 & 2 & 1 & 2 & 1 & 2 & PM & Remus & 2007 & success & explicit \\
\hline 830 & Flexible structure & 1 & 1 & 1 & 1 & 4 & 23 & PM & Remus & 2007 & success & explicit \\
\hline 831 & Change management & 1 & 2 & 1 & 2 & 1 & 2 & $\mathrm{PM}$ & Remus & 2007 & success & explicit \\
\hline 832 & Requirements analysis & 4 & 23 & 4 & 23 & 4 & 23 & PM & Remus & 2007 & success & explicit \\
\hline 833 & Project management & 4 & 17 & 4 & 17 & 4 & 17 & PM & Remus & 2007 & success & explicit \\
\hline
\end{tabular}




\begin{tabular}{|c|c|c|c|c|c|c|c|c|c|c|c|c|}
\hline 834 & Prototyping & 4 & 23 & 4 & 23 & 4 & 23 & PM & Remus & 2007 & success & explicit \\
\hline 835 & Team competencies and skills & 4 & 24 & 4 & 24 & 4 & 24 & PM & Remus & 2007 & success & explicit \\
\hline 836 & $\begin{array}{l}\text { Defining the portal architec- } \\
\text { ture }\end{array}$ & 4 & 23 & 4 & 23 & 6 & 27 & PM & Remus & 2007 & success & explicit \\
\hline 837 & $\begin{array}{l}\text { Business process reengineer- } \\
\text { ing }\end{array}$ & 4 & 19 & 4 & 19 & 4 & 19 & $\mathrm{PM}$ & Remus & 2007 & success & explicit \\
\hline 838 & Portal engineering roadmap & 4 & 23 & 4 & 23 & 4 & 23 & $\mathrm{PM}$ & Remus & 2007 & success & explicit \\
\hline 839 & $\begin{array}{l}\text { Project monitoring and con- } \\
\text { trolling }\end{array}$ & 4 & 22 & 4 & 22 & 4 & 22 & $\mathrm{PM}$ & Remus & 2007 & success & explicit \\
\hline 840 & Team competencies and skills & 4 & 24 & 4 & 24 & 4 & 24 & $\mathrm{PM}$ & Remus & 2007 & success & explicit \\
\hline 841 & Project management & 4 & 17 & 4 & 17 & 4 & 17 & $\mathrm{PM}$ & Remus & 2007 & success & explicit \\
\hline 842 & Project champion & 4 & 24 & 4 & 24 & 4 & 24 & $\mathrm{PM}$ & Remus & 2007 & success & explicit \\
\hline 843 & Careful package selection & 4 & 23 & 4 & 23 & 4 & 23 & PM & Remus & 2007 & success & explicit \\
\hline 844 & $\begin{array}{l}\text { Business Process Reengineer- } \\
\text { ing }\end{array}$ & 4 & 19 & 4 & 19 & 4 & 19 & PM & Remus & 2007 & success & explicit \\
\hline 845 & Minimal customization & 4 & 19 & 2 & 6 & 3 & 8 & PM & Remus & 2007 & success & explicit \\
\hline 846 & Architecture choice & 4 & 23 & 4 & 23 & 6 & 28 & PM & & 2007 & success & explicit \\
\hline 847 & User acceptance & 2 & 6 & 2 & 6 & 2 & 6 & $\mathrm{PM}$ & & 2007 & success & explicit \\
\hline 848 & Top management support & 3 & 15 & 3 & 15 & 3 & 15 & PM & Remus & 2007 & success & explicit \\
\hline 849 & $\begin{array}{l}\text { Strong communication in- } \\
\text { wards and outwards }\end{array}$ & 3 & 10 & 3 & 10 & 3 & 10 & PM & Remus & 2007 & success & explicit \\
\hline 850 & User training and education & 3 & 11 & 3 & 11 & 3 & 11 & PM & Remus & 2007 & success & explicit \\
\hline 851 & Dedicated resources & 3 & 13 & 3 & 13 & 3 & 13 & PM & Remus & 2007 & success & explicit \\
\hline 852 & Top management support & 3 & 15 & 3 & 15 & 3 & 15 & PM & Remus & 2007 & success & explicit \\
\hline 853 & $\begin{array}{l}\text { Interdepartmental communica- } \\
\text { tion }\end{array}$ & 3 & 11 & 3 & 11 & 3 & 11 & PM & Remus & 2007 & success & explicit \\
\hline 854 & Vendor support & 3 & 12 & 3 & 12 & 3 & 12 & PM & Remus & 2007 & success & explicit \\
\hline 855 & Dedicated resources & 3 & 13 & 3 & 13 & 3 & 13 & PM & Remus & 2007 & success & explicit \\
\hline 856 & Use of steering committee & 3 & 15 & 3 & 15 & 6 & 28 & PM & Remus & 2007 & success & explicit \\
\hline 857 & User training on software & 3 & 11 & 3 & 11 & 3 & 11 & PM & Remus & 2007 & success & explicit \\
\hline 858 & $\begin{array}{l}\text { Education on new business } \\
\text { processes }\end{array}$ & 3 & 11 & 3 & 11 & 3 & 11 & PM & Remus & 2007 & success & explicit \\
\hline 859 & Partnership with vendor & 3 & 12 & 3 & 12 & 3 & 12 & PM & Remus & 2007 & success & explicit \\
\hline 860 & Use of vendors' tools & 3 & 12 & 3 & 12 & 3 & 12 & $\mathrm{PM}$ & Remus & 2007 & success & explicit \\
\hline 861 & Clear goals and objectives & 5 & 25 & 5 & 25 & 5 & 25 & $\mathrm{PM}$ & Remus & 2007 & success & explicit \\
\hline 862 & Portal strategy & 5 & 26 & 5 & 26 & 5 & 26 & $\mathrm{PM}$ & Remus & 2007 & success & explicit \\
\hline 863 & Interdepartmental cooperation & 5 & 26 & 5 & 26 & 3 & 11 & $\mathrm{PM}$ & Remus & 2007 & success & explicit \\
\hline 864 & Clear goals and objectives & 5 & 25 & 5 & 25 & 5 & 25 & $\mathrm{PM}$ & Remus & 2007 & success & explicit \\
\hline 865 & Management of expectations & 5 & 25 & 5 & 25 & 5 & 25 & $\mathrm{PM}$ & Remus & 2007 & success & explicit \\
\hline 866 & $\begin{array}{l}\text { Process and application inte- } \\
\text { gration }\end{array}$ & 6 & 27 & 6 & 27 & 6 & 27 & PM & Remus & 2007 & success & explicit \\
\hline 867 & Organizational culture & 6 & 28 & 0 & 28 & 6 & 28 & $\mathrm{PM}$ & Remus & 2007 & success & explicit \\
\hline 868 & Data analysis and conversion & 6 & 27 & 6 & 27 & 6 & 27 & $\mathrm{PM}$ & Remus & 2007 & success & explicit \\
\hline 869 & $\begin{array}{l}\text { Selection of the appropriate } \\
\text { portal package }\end{array}$ & 7 & 33 & 4 & 23 & 6 & 27 & PM & Remus & 2007 & success & explicit \\
\hline 870 & Portal design & 7 & 29 & 1 & 29 & 7 & 29 & $\mathrm{PM}$ & Remus & 2007 & success & explicit \\
\hline 871 & $\begin{array}{l}\text { Use of a champion in a signifi- } \\
\text { cant role }\end{array}$ & 4 & 24 & 4 & 24 & 4 & 24 & PM & Bradley & 2005 & success & implicit \\
\hline 872 & $\begin{array}{l}\text { High training quality and } \\
\text { spending a lot of time in train- } \\
\text { ing }\end{array}$ & 3 & 11 & 3 & 11 & 3 & 11 & PM & Bradley & 2005 & success & implicit \\
\hline 873 & Use of Consultants & 3 & 10 & 3 & 10 & 3 & 10 & $\mathrm{PM}$ & Bradley & 2005 & success & implicit \\
\hline
\end{tabular}




\begin{tabular}{|c|c|c|c|c|c|c|c|c|c|c|c|c|}
\hline 874 & $\begin{array}{l}\text { Use of a steering committee } \\
\text { headed by the CEO to review } \\
\text { the project }\end{array}$ & 3 & 15 & 3 & 15 & 6 & 28 & PM & Bradley & 2005 & success & implicit \\
\hline 875 & $\begin{array}{l}\text { Project Manager ERP Experi- } \\
\text { ence }\end{array}$ & 7 & 30 & 7 & 30 & 7 & 30 & PM & Bradley & 2005 & success & implicit \\
\hline 876 & Organizational change & 1 & 2 & 1 & 2 & 1 & 2 & PM & Ram et al. & 2013 & success & implicit \\
\hline 877 & full-time project manager & 4 & 17 & 4 & 17 & 4 & 17 & $\mathrm{PM}$ & Ram et al. & 2013 & success & implicit \\
\hline 878 & $\begin{array}{l}\text { Business process reengineer- } \\
\text { ing }\end{array}$ & 4 & 19 & 4 & 19 & 4 & 19 & PM & Ram et al. & 2013 & success & implicit \\
\hline 879 & Project champion & 4 & 24 & 4 & 24 & 4 & 24 & PM & Ram et al. & 2013 & success & implicit \\
\hline 880 & $\begin{array}{l}\text { BPR and minimum customisa- } \\
\text { tion }\end{array}$ & 4 & 19 & 4 & 19 & 3 & 8 & PM & Ram et al. & 2013 & success & explicit \\
\hline 881 & $\begin{array}{l}\text { Teamwork / Project team } \\
\text { composition, competence and } \\
\text { compensation }\end{array}$ & 4 & 24 & 4 & 24 & 4 & 24 & PM & Ram et al. & 2013 & success & explicit \\
\hline 882 & Careful package selection & 4 & 23 & 4 & 23 & 4 & 23 & $\mathrm{PM}$ & Ram et al. & 2013 & success & explicit \\
\hline 883 & Charismatic leadership & 4 & 17 & 4 & 17 & 4 & 17 & $\mathrm{PM}$ & Ram et al. & 2013 & success & explicit \\
\hline 884 & $\begin{array}{l}\text { Strict monitoring of imple- } \\
\text { mentation schedule and costs }\end{array}$ & 4 & 21 & 4 & 22 & 4 & 22 & PM & Ram et al. & 2013 & success & implicit \\
\hline 885 & $\begin{array}{l}\text { Regular project status meet- } \\
\text { ings }\end{array}$ & 4 & 22 & 4 & 22 & 4 & 23 & PM & Ram et al. & 2013 & success & implicit \\
\hline 886 & $\begin{array}{l}\text { establish implementation strat- } \\
\text { egies and systematic guide- } \\
\text { lines }\end{array}$ & 4 & 23 & 4 & 23 & 4 & 23 & PM & Ram et al. & 2013 & success & implicit \\
\hline 887 & Leadership & 3 & 15 & 3 & 15 & 4 & 17 & PM & Ram et al. & 2013 & success & implicit \\
\hline 888 & Vendor support & 3 & 12 & 3 & 12 & 3 & 12 & PM & Ram et al. & 2013 & success & implicit \\
\hline 889 & Providing training to staff & 3 & 11 & 3 & 11 & 3 & 11 & PM & Ram et al. & 2013 & success & implicit \\
\hline 890 & external expertise & 3 & 10 & 3 & 10 & 3 & 10 & PM & Ram et al. & 2013 & success & implicit \\
\hline 891 & $\begin{array}{l}\text { Strong Communication out- } \\
\text { wards }\end{array}$ & 3 & 10 & 3 & 10 & 3 & 10 & PM & Ram et al. & 2013 & success & explicit \\
\hline 892 & $\begin{array}{l}\text { Training employee / user } \\
\text { training and education / job re- } \\
\text { design }\end{array}$ & 3 & 11 & 3 & 11 & 3 & 11 & PM & Ram et al. & 2013 & success & explicit \\
\hline 893 & ERP vendor support & 3 & 12 & 3 & 12 & 3 & 12 & $\mathrm{PM}$ & Ram et al. & 2013 & success & explicit \\
\hline 894 & $\begin{array}{l}\text { Consultant quality / use of } \\
\text { consultants }\end{array}$ & 3 & 10 & 3 & 10 & 3 & 10 & PM & Ram et al. & 2013 & success & explicit \\
\hline 895 & $\begin{array}{l}\text { Sustained (top) management } \\
\text { support / commitment }\end{array}$ & 3 & 15 & 3 & 15 & 3 & 15 & PM & Ram et al. & 2013 & success & explicit \\
\hline 896 & $\begin{array}{l}\text { User involvement, participa- } \\
\text { tion and support }\end{array}$ & 3 & 8 & 3 & 8 & 3 & 8 & PM & Ram et al. & 2013 & success & explicit \\
\hline 897 & $\begin{array}{l}\text { training programs that are tai- } \\
\text { lored to build users' confi- } \\
\text { dence when using the ERP } \\
\text { system }\end{array}$ & 3 & 11 & 3 & 11 & 3 & 11 & PM & Ram et al. & 2013 & success & implicit \\
\hline 898 & $\begin{array}{l}\text { training programs that are sub- } \\
\text { stantially improve the level of } \\
\text { understanding of users }\end{array}$ & 3 & 11 & 3 & 11 & 3 & 11 & PM & Ram et al. & 2013 & success & implicit \\
\hline 899 & $\begin{array}{l}\text { training programs that are of } \\
\text { adequate length and detail }\end{array}$ & 3 & 11 & 3 & 11 & 3 & 11 & PM & Ram et al. & 2013 & success & implicit \\
\hline 900 & $\begin{array}{l}\text { integration of ERP with part- } \\
\text { ner organisations' information } \\
\text { systems }\end{array}$ & 3 & 12 & 7 & 33 & 3 & 12 & PM & Ram et al. & 2013 & success & implicit \\
\hline 901 & Having a business vision & 5 & 26 & 5 & 26 & 6 & 28 & $\mathrm{PM}$ & Ram et al. & 2013 & success & implicit \\
\hline 902 & Formal project plan/schedule & 5 & 25 & 4 & 23 & 4 & 23 & $\mathrm{PM}$ & Ram et al. & 2013 & success & explicit \\
\hline 903 & $\begin{array}{l}\text { Carefully defined scope of the } \\
\text { ERP project }\end{array}$ & 5 & 25 & 4 & 23 & 4 & 23 & PM & Ram et al. & 2013 & success & implicit \\
\hline
\end{tabular}




\begin{tabular}{|c|c|c|c|c|c|c|c|c|c|c|c|c|}
\hline 904 & Organisational Readiness & 6 & 28 & 6 & 28 & 6 & 28 & $\mathrm{PM}$ & Ram et al. & 2013 & success & implicit \\
\hline 905 & $\begin{array}{l}\text { Data analysis,conversion and } \\
\text { integrity }\end{array}$ & 6 & 27 & 6 & 27 & 6 & 27 & $\mathrm{PM}$ & Ram et al. & 2013 & success & explicit \\
\hline 906 & Implementation quality & 7 & 29 & 7 & 29 & 4 & 22 & PM & Ram et al. & 2013 & success & implicit \\
\hline 907 & Implementation Approach & 4 & 23 & 4 & 23 & 4 & 23 & BPI & $\begin{array}{l}\text { McLean } \\
\text { and Antony }\end{array}$ & 2014 & failure & explicit \\
\hline 908 & Project Management & 4 & 17 & 4 & 17 & 4 & 17 & BPI & $\begin{array}{l}\text { McLean } \\
\text { and Antony }\end{array}$ & 2014 & failure & explicit \\
\hline 909 & The Management Leadership & 3 & 15 & 4 & 17 & 4 & 17 & BPI & $\begin{array}{l}\text { McLean } \\
\text { and Antony }\end{array}$ & 2014 & failure & explicit \\
\hline 910 & Training & 3 & 11 & 3 & 11 & 3 & 11 & BPI & $\begin{array}{l}\text { McLean } \\
\text { and Antony }\end{array}$ & 2014 & failure & explicit \\
\hline 911 & Employee Involvement Levels & 3 & 9 & 3 & 11 & 6 & 28 & BPI & $\begin{array}{l}\text { McLean } \\
\text { and Antony }\end{array}$ & 2014 & failure & explicit \\
\hline 912 & Feedback and Results & 3 & 11 & 3 & 11 & 3 & 11 & BPI & $\begin{array}{l}\text { McLean } \\
\text { and Antony }\end{array}$ & 2014 & failure & explicit \\
\hline 913 & Motives and Expectations & 5 & 25 & 5 & 25 & 5 & 25 & BPI & $\begin{array}{l}\text { McLean } \\
\text { and Antony }\end{array}$ & 2014 & failure & explicit \\
\hline 914 & $\begin{array}{l}\text { Organizational Culture and } \\
\text { Evironment }\end{array}$ & 6 & 28 & 6 & 28 & 6 & 28 & BPI & $\begin{array}{l}\text { McLean } \\
\text { and Antony }\end{array}$ & 2014 & failure & explicit \\
\hline 915 & risk disposition & 1 & 5 & 1 & 5 & 1 & 2 & BPR & $\begin{array}{l}\text { Paper } \\
\text { and Chang }\end{array}$ & 2005 & success & explicit \\
\hline 916 & creativity & 1 & 1 & 1 & 1 & 1 & 2 & BPR & $\begin{array}{l}\text { Paper } \\
\text { and Chang }\end{array}$ & 2005 & success & implicit \\
\hline 917 & openness to change & 1 & 2 & 1 & 2 & 1 & 2 & BPR & $\begin{array}{l}\text { Paper } \\
\text { and Chang }\end{array}$ & 2005 & success & implicit \\
\hline 918 & empowerment & 1 & 5 & 3 & 11 & 3 & 9 & BPR & $\begin{array}{l}\text { Paper } \\
\text { and Chang }\end{array}$ & 2005 & success & explicit \\
\hline 919 & $\begin{array}{l}\text { help people understand the } \\
\text { reasons for change }\end{array}$ & 1 & 2 & 1 & 2 & 1 & 2 & BPR & $\begin{array}{l}\text { Paper } \\
\text { and Chang }\end{array}$ & 2005 & success & implicit \\
\hline 920 & vision flexibility & 1 & 3 & 5 & 26 & 5 & 26 & BPR & $\begin{array}{l}\text { Paper } \\
\text { and Chang }\end{array}$ & 2005 & success & explicit \\
\hline 921 & teaming & 4 & 24 & 4 & 24 & 3 & 14 & BPR & $\begin{array}{l}\text { Paper } \\
\text { and Chang }\end{array}$ & 2005 & success & explicit \\
\hline 922 & knowledge & 4 & 16 & 3 & 11 & 4 & 16 & BPR & $\begin{array}{l}\text { Paper } \\
\text { and Chang }\end{array}$ & 2005 & success & implicit \\
\hline 923 & ownership & 4 & 17 & 4 & 17 & 6 & 28 & BPR & $\begin{array}{l}\text { Paper } \\
\text { and Chang }\end{array}$ & 2005 & success & explicit \\
\hline 924 & team work & 4 & 24 & 4 & 24 & 3 & 14 & BPR & $\begin{array}{l}\text { Paper } \\
\text { and Chang }\end{array}$ & 2005 & success & implicit \\
\hline 925 & $\begin{array}{l}\text { Methodology is critical to ef- } \\
\text { fectively dealing with the } \\
\text { scope and complexities in- } \\
\text { volved in change }\end{array}$ & 4 & 23 & 4 & 23 & 4 & 23 & BPR & $\begin{array}{l}\text { Paper } \\
\text { and Chang }\end{array}$ & 2005 & success & implicit \\
\hline 926 & developing process workers & 4 & 16 & 3 & 11 & 3 & 11 & BPR & $\begin{array}{l}\text { Paper } \\
\text { and Chang }\end{array}$ & 2005 & success & implicit \\
\hline 927 & continuous monitoring & 4 & 21 & 4 & 21 & 4 & 21 & BPR & $\begin{array}{l}\text { Paper } \\
\text { and Chang }\end{array}$ & 2005 & success & explicit \\
\hline 928 & appropriate guiding principles & 2 & 7 & 4 & 23 & 5 & 25 & BPR & $\begin{array}{l}\text { Paper } \\
\text { and Chang }\end{array}$ & 2005 & success & explicit \\
\hline 929 & graphical process map & 2 & 7 & 4 & 20 & 4 & 23 & BPR & $\begin{array}{l}\text { Paper } \\
\text { and Chang }\end{array}$ & 2005 & success & explicit \\
\hline 930 & top-management support & 3 & 15 & 3 & 15 & 3 & 15 & BPR & $\begin{array}{l}\text { Paper } \\
\text { and Chang }\end{array}$ & 2005 & success & explicit \\
\hline 931 & organizational learning & 3 & 11 & 3 & 11 & 3 & 11 & BPR & $\begin{array}{l}\text { Paper } \\
\text { and Chang }\end{array}$ & 2005 & success & explicit \\
\hline
\end{tabular}




\begin{tabular}{|c|c|c|c|c|c|c|c|c|c|c|c|c|}
\hline 932 & $\begin{array}{l}\text { compensation and reward sys- } \\
\text { tems }\end{array}$ & 3 & 14 & 3 & 14 & 3 & 14 & BPR & $\begin{array}{l}\text { Paper } \\
\text { and Chang }\end{array}$ & 2005 & success & explicit \\
\hline 933 & information sharing & 3 & 11 & 3 & 11 & 3 & 11 & BPR & $\begin{array}{l}\text { Paper } \\
\text { and Chang }\end{array}$ & 2005 & success & explicit \\
\hline 934 & resources & 3 & 13 & 3 & 13 & 3 & 13 & BPR & $\begin{array}{l}\text { Paper } \\
\text { and Chang }\end{array}$ & 2005 & success & explicit \\
\hline 935 & politics resolution & 3 & 15 & 3 & 15 & 3 & 15 & BPR & $\begin{array}{l}\text { Paper } \\
\text { and Chang }\end{array}$ & 2005 & success & explicit \\
\hline 936 & training & 3 & 11 & 3 & 11 & 3 & 11 & BPR & $\begin{array}{l}\text { Paper } \\
\text { and Chang }\end{array}$ & 2005 & success & explicit \\
\hline 937 & education & 3 & 11 & 3 & 11 & 3 & 11 & BPR & $\begin{array}{l}\text { Paper } \\
\text { and Chang }\end{array}$ & 2005 & success & explicit \\
\hline 938 & challenging work & 3 & 14 & 3 & 14 & 3 & 9 & BPR & $\begin{array}{l}\text { Paper } \\
\text { and Chang }\end{array}$ & 2005 & success & implicit \\
\hline 939 & $\begin{array}{l}\text { people's view of the IT devel- } \\
\text { opment process }\end{array}$ & 3 & 9 & 3 & 9 & 7 & 29 & BPR & $\begin{array}{l}\text { Paper } \\
\text { and Chang }\end{array}$ & 2005 & success & implicit \\
\hline 940 & $\begin{array}{l}\text { extracting the knowledge } \\
\text { gained by the process workers }\end{array}$ & 3 & 11 & 3 & 11 & 2 & 7 & BPR & $\begin{array}{l}\text { Paper } \\
\text { and Chang }\end{array}$ & 2005 & success & implicit \\
\hline 941 & $\begin{array}{l}\text { top-down vision tempered } \\
\text { with involvement from pro- } \\
\text { cess workers }\end{array}$ & 3 & 9 & 3 & 11 & 3 & 11 & BPR & $\begin{array}{l}\text { Paper } \\
\text { and Chang }\end{array}$ & 2005 & success & implicit \\
\hline 942 & $\begin{array}{l}\text { proactively sell the vision to } \\
\text { key stakeholders before imple- } \\
\text { menting change }\end{array}$ & 3 & 8 & 3 & 8 & 3 & 8 & BPR & $\begin{array}{l}\text { Paper } \\
\text { and Chang }\end{array}$ & 2005 & success & implicit \\
\hline 943 & customer support & 3 & 8 & 3 & 8 & 2 & 6 & BPR & $\begin{array}{l}\text { Paper } \\
\text { and Chang }\end{array}$ & 2005 & success & explicit \\
\hline 944 & IT belief system & 3 & 15 & 6 & 27 & 6 & 27 & BPR & $\begin{array}{l}\text { Paper } \\
\text { and Chang }\end{array}$ & 2005 & success & explicit \\
\hline 945 & vision communication & 3 & 11 & 5 & 26 & 3 & 11 & BPR & $\begin{array}{l}\text { Paper } \\
\text { and Chang }\end{array}$ & 2005 & success & explicit \\
\hline 946 & vision development & 5 & 26 & 5 & 26 & 5 & 26 & BPR & $\begin{array}{l}\text { Paper } \\
\text { and Chang }\end{array}$ & 2005 & success & explicit \\
\hline 947 & vision deployment & 5 & 26 & 5 & 26 & 5 & 26 & BPR & $\begin{array}{l}\text { Paper } \\
\text { and Chang }\end{array}$ & 2005 & success & explicit \\
\hline 948 & direction & 6 & 28 & 6 & 28 & 6 & 28 & BPR & $\begin{array}{l}\text { Paper } \\
\text { and Chang }\end{array}$ & 2005 & success & explicit \\
\hline 949 & IT architecture & 6 & 27 & 6 & 27 & 6 & 27 & BPR & $\begin{array}{l}\text { Paper } \\
\text { and Chang }\end{array}$ & 2005 & success & explicit \\
\hline 950 & $\begin{array}{l}\text { people's role in the IT devel- } \\
\text { opment process }\end{array}$ & 7 & 29 & 7 & 30 & 7 & 29 & BPR & $\begin{array}{l}\text { Paper } \\
\text { and Chang }\end{array}$ & 2005 & success & implicit \\
\hline 951 & IT knowledge & 7 & 29 & 7 & 29 & 7 & 29 & BPR & $\begin{array}{l}\text { Paper } \\
\text { and Chang }\end{array}$ & 2005 & success & explicit \\
\hline 952 & Origin of idea & 4 & 23 & 4 & 23 & 4 & 23 & PM & Shenhar et al. & 2002 & success & explicit \\
\hline 953 & Project milestones & 4 & 22 & 4 & 23 & 4 & 23 & PM & Shenhar et al. & 2002 & success & explicit \\
\hline 954 & Planning and control methods & 4 & 21 & 4 & 23 & 4 & 23 & PM & Shenhar et al. & 2002 & success & explicit \\
\hline 955 & Design cycles & 4 & 23 & 4 & 23 & 4 & 23 & $\mathrm{PM}$ & Shenhar et al. & 2002 & success & explicit \\
\hline 956 & Design techniques & 4 & 20 & 4 & 23 & 4 & 23 & PM & Shenhar et al. & 2002 & success & explicit \\
\hline 957 & Quality management & 4 & 17 & 4 & 17 & 4 & 22 & PM & Shenhar et al. & 2002 & success & explicit \\
\hline 958 & Project team & 4 & 24 & 4 & 24 & 4 & 24 & PM & Shenhar et al. & 2002 & success & explicit \\
\hline 959 & Design reviews & 4 & 22 & 4 & 22 & 4 & 23 & PM & Shenhar et al. & 2002 & success & explicit \\
\hline 960 & Management policy & 4 & 17 & 4 & 17 & 4 & 17 & PM & Shenhar et al. & 2002 & success & explicit \\
\hline 961 & $\begin{array}{l}\text { Work Breakdown Structure } \\
\text { (WBS) }\end{array}$ & 2 & 7 & 4 & 23 & 4 & 23 & PM & Shenhar et al. & 2002 & success & explicit \\
\hline 962 & Documentation & 2 & 7 & 2 & 7 & 3 & 11 & PM & Shenhar et al. & 2002 & success & explicit \\
\hline 963 & Resource sharing & 3 & 13 & 3 & 13 & 3 & 13 & PM & Shenhar et al. & 2002 & success & explicit \\
\hline
\end{tabular}




\begin{tabular}{|c|c|c|c|c|c|c|c|c|c|c|c|c|}
\hline 964 & Customer participation & 3 & 8 & 3 & 8 & 3 & 8 & PM & Shenhar et al. & 2002 & success & explicit \\
\hline 965 & Design considerations & 5 & 25 & 4 & 23 & 4 & 23 & $\mathrm{PM}$ & henhar et al. & 2002 & success & explicit \\
\hline 966 & ormal procedures & 6 & 28 & 6 & 28 & 6 & 28 & $\mathrm{PM}$ & henhar et al. & 2002 & success & explicit \\
\hline 967 & Organizational structure & 6 & 28 & 6 & 28 & 6 & 28 & PM & henhar et al. & 2002 & success & explicit \\
\hline 968 & PM autonomy & 6 & 28 & 4 & 23 & 6 & 28 & PM & henhar et al. & 2002 & success & explicit \\
\hline 969 & Formal contracts & 6 & 28 & 6 & 28 & 6 & 28 & PM & Shenhar et al. & 2002 & success & explicit \\
\hline 970 & $\begin{array}{l}\text { Integrated Master Schedule } \\
\text { (IMS) }\end{array}$ & 4 & 23 & 4 & 23 & 4 & 23 & PM & Allen at el. & 2014 & success & explicit \\
\hline 971 & Control Account Planning & 4 & 23 & 3 & 13 & 4 & 22 & $\mathrm{PM}$ & Allen at el. & 2014 & success & explicit \\
\hline 972 & Controlling and Monitoring & 4 & 21 & 4 & 21 & 4 & 22 & PM & llen at el. & 2014 & success & explicit \\
\hline 973 & $\begin{array}{l}\text { Work Breakdown Structure } \\
\text { (WBS) }\end{array}$ & 2 & 7 & 4 & 23 & 4 & 23 & PM & Allen at el. & 2014 & success & explicit \\
\hline 974 & lder Partnership & 3 & 8 & 3 & 12 & 3 & 8 & PM & llen at el. & 2014 & success & explicit \\
\hline 975 & Lessons Learned & 3 & 11 & 3 & 11 & 3 & 11 & $\mathrm{PM}$ & llen at el. & 2014 & success & explicit \\
\hline 976 & Team Building & 3 & 14 & 3 & 14 & 3 & 14 & $\mathrm{PM}$ & llen at el. & 2014 & success & explicit \\
\hline 977 & Integrated Master Plan (IMP) & 5 & 25 & 4 & 23 & 4 & 23 & $\mathrm{PM}$ & llen at el. & 14 & success & explicit \\
\hline 978 & Organizational Influence & 6 & 28 & 6 & 28 & 6 & 28 & $\mathrm{PM}$ & & 14 & uccess & explicit \\
\hline 979 & Organizational Structure & 6 & 28 & 6 & 28 & 6 & 28 & PM & & 14 & uccess & licit \\
\hline 980 & $\begin{array}{l}\text { Orga } \\
\text { Struc }\end{array}$ & 6 & 28 & 4 & 23 & 4 & 23 & PM & Allen at el. & 2014 & uccess & xplicit \\
\hline 981 & $\begin{array}{l}\text { Respc } \\
\text { Matri }\end{array}$ & 6 & 28 & 6 & 28 & 4 & 23 & PM & Allen atel & 2014 & uccess & xplicit \\
\hline 982 & & 1 & 3 & 4 & 22 & 4 & 25 & & & & & \\
\hline 983 & & 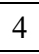 & $23 \mid$ & 4 & 23 & 4 & 23 & & & 2015 & success & \\
\hline 984 & Team & 4 & 24 & 4 & 24 & 4 & 24 & & & 15 & success & licit \\
\hline 985 & Deter & 4 & \begin{tabular}{|l|l} 
& \\
\end{tabular} & 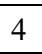 & 23 & 4 & 22 & PM & & 15 & success & licit \\
\hline 986 & abilit & 4 & & 4 & 17 & 4 & 17 & $\mathrm{M}$ & & 2015 & success & licit \\
\hline 987 & defini & 4 & 23 & & 23 & 4 & 23 & $\mathrm{M}$ & & 2015 & success & explicit \\
\hline 988 & $\begin{array}{l}\text { acc } \\
\text { pro }\end{array}$ & 4 & 17 & 3 & 9 & 4 & 17 & PM & Besteiro et al. & 2015 & uccess & xplicit \\
\hline 989 & $\begin{array}{l}\text { Defining the scope of the pro- } \\
\text { ject }\end{array}$ & 4 & 23 & 4 & 23 & 4 & 23 & PM & Besteiro et al. & 2015 & success & explicit \\
\hline 990 & Planning & & & & 23 & 4 & 23 & PM & & 2015 & uccess & licit \\
\hline 991 & Communicating the $p$ & 3 & \begin{tabular}{|l|l|}
11 & \\
\end{tabular} & 2 & 11 & 3 & 11 & PM & Patairato1 & 2015 & success & explicit \\
\hline 992 & the mission of the & 3 & 9 & 3 & 9 & 5 & 25 & PM & Besteiro et al. & 2015 & success & explicit \\
\hline 993 & Project monitoring meetings & & & & 11 & 4 & -5 & PM & & 2015 & success & explicit \\
\hline 994 & Feedback meetings & & 11 & & 11 & 3 & 11 & PM & & 2015 & 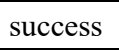 & explicit \\
\hline 995 & $\begin{array}{l}\text { Planned vs. actual bu } \\
\text { ation }\end{array}$ & 3 & 13 & 4 & 22 & 4 & 22 & PM & Besteiro et al. & 2015 & success & explicit \\
\hline 996 & Commitment from the team & 3 & | & & F & 3 & 14 & $\mathrm{PM}$ & & 2015 & suctess & explicit \\
\hline 997 & Meeting the budget & 3 & & & 13 & 3 & 13 & $\mathrm{PM}$ & Besteiro et al. & 2015 & success & explicit \\
\hline 998 & Ability to communicate & 3 & 10 & & 10 & 3 & 10 & PM & Besteiro et al. & 2015 & success & explicit \\
\hline 999 & Goals with a realistic objective & & \begin{tabular}{|l|l|l|}
25 & \\
\end{tabular} & & 25 & 5 & 25 & PM & Besteiro et al. & 2015 & uccess & explicit \\
\hline 1000 & $\begin{array}{l}\text { Planned vs. actual benefit vari- } \\
\text { ation }\end{array}$ & 5 & 25 & 4 & 22 & 4 & 22 & PM & Besteiro et al. & 2015 & uccess & xplicit \\
\hline 1001 & $\begin{array}{l}\text { Planned vs. actual deadline } \\
\text { variation }\end{array}$ & 5 & 25 & 4 & 22 & 4 & 22 & PM & Besteiro et al. & 2015 & success & explicit \\
\hline 1002 & $\begin{array}{l}\text { defining realistic goals and o } \\
\text { jectives and team qualificatio }\end{array}$ & 3 & 25 & 5 & 25 & 3 & 9 & PM & Besteiro et al. & 2015 & success & explicit \\
\hline 1003 & $\begin{array}{l}\text { Indicating roles and responsi- } \\
\text { bilities }\end{array}$ & 0 & 20 & 0 & 28 & 6 & 20 & PM & Besteiro et al. & 2015 & success & explicit \\
\hline
\end{tabular}




\begin{tabular}{|c|c|c|c|c|c|c|c|c|c|c|c|c|}
\hline 1004 & $\begin{array}{l}\text { indicating roles and responsi- } \\
\text { bilities }\end{array}$ & 6 & 28 & 6 & 28 & 6 & 28 & PM & Besteiro et al. & 2015 & success & explicit \\
\hline 1005 & Project Management & 4 & 17 & 4 & 17 & 4 & 17 & BPR & Dezdar & 2012 & success & explicit \\
\hline 1006 & Top Management Support & 3 & 15 & 3 & 15 & 3 & 15 & BPR & Dezdar & 2012 & uccess & xplicit \\
\hline 1007 & $\begin{array}{l}\text { Entreprise-Wide Communica- } \\
\text { tion }\end{array}$ & 3 & 11 & 3 & 11 & 3 & 11 & BPR & Dezdar & 2012 & success & explicit \\
\hline 1008 & User Training and Education & 3 & 11 & 3 & 11 & 3 & 8 & BPR & Dezdar & 2012 & success & explicit \\
\hline 1009 & ERP Vendor Support & 3 & 12 & 3 & 12 & 3 & 12 & $\mathrm{BPR}$ & Dezdar & 2012 & success & explicit \\
\hline 1010 & Risk Management & 1 & 5 & 1 & 5 & 1 & 5 & PM & $\begin{array}{l}\text { Gomes } \\
\text { and Romão }\end{array}$ & 2016 & success & explicit \\
\hline 1011 & Scope Control & 4 & 22 & 4 & 22 & 4 & 23 & PM & $\begin{array}{l}\text { Gomes } \\
\text { and Romão }\end{array}$ & 2016 & success & explicit \\
\hline 1012 & Business Opportunity & 2 & 6 & 2 & 6 & 5 & 25 & PM & $\begin{array}{l}\text { Gomes } \\
\text { and Romão }\end{array}$ & 2016 & success & explicit \\
\hline 1013 & Market Impact & 2 & 6 & 2 & 6 & 2 & 6 & PM & $\begin{array}{l}\text { Gomes } \\
\text { and Romão }\end{array}$ & 2016 & success & explicit \\
\hline 1014 & Top Management Support & 3 & 15 & 3 & 15 & 3 & 15 & PM & $\begin{array}{l}\text { Gomes } \\
\text { and Romão }\end{array}$ & 2016 & success & explicit \\
\hline 1015 & Team Engagement & 3 & 14 & 3 & 14 & 3 & 14 & $\mathrm{PM}$ & $\begin{array}{l}\text { Gomes } \\
\text { and Romão }\end{array}$ & 2016 & success & explicit \\
\hline 1016 & Resource Availability & 3 & 13 & 3 & 13 & 3 & 13 & PM & $\begin{array}{l}\text { Gomes } \\
\text { and Romão }\end{array}$ & 2016 & success & explicit \\
\hline 1017 & Financial Resources & 3 & 13 & 3 & 13 & 3 & 13 & PM & $\begin{array}{l}\text { mes } \\
\text { d Romão }\end{array}$ & 2016 & success & explicit \\
\hline 1018 & Effective project management & 4 & 17 & 4 & 17 & 4 & 17 & PM & $\begin{array}{l}\text { ler } \\
\text { Horan }\end{array}$ & 2007 & success & explicit \\
\hline 1019 & $\begin{array}{l}\text { Project personnel } \\
\text { knowledge/skills }\end{array}$ & 4 & 16 & 4 & 24 & 4 & 16 & PM & $\begin{array}{l}\text { Fowler } \\
\text { and Horan }\end{array}$ & 2007 & success & explicit \\
\hline 1020 & $\begin{array}{l}\text { Lack of effective project man- } \\
\text { agement }\end{array}$ & 4 & 17 & 4 & 17 & 4 & 17 & $\mathrm{PM}$ & $\begin{array}{l}\text { Fowler } \\
\text { and Horan }\end{array}$ & 2007 & failure & explicit \\
\hline 1021 & $\begin{array}{l}\text { Lack required } \\
\text { knowledge/skills in the project } \\
\text { personnel }\end{array}$ & 4 & 16 & 4 & 24 & 4 & 16 & PM & $\begin{array}{l}\text { Fowler } \\
\text { and Horan }\end{array}$ & 2007 & failure & explicit \\
\hline 1022 & User : & 2 & 6 & 2 & 6 & 2 & 6 & PM & $\begin{array}{l}\text { Fowler } \\
\text { and Horan }\end{array}$ & 2007 & success & explicit \\
\hline 1023 & Top management commitment & 3 & 15 & 3 & 15 & 3 & 15 & PM & $\begin{array}{l}\text { Fowler } \\
\text { and Horan }\end{array}$ & 2007 & success & explicit \\
\hline 1024 & project team commitment & 3 & 9 & 3 & 9 & 3 & 14 & $\mathrm{PM}$ & $\begin{array}{l}\text { Fowler } \\
\text { and Horan }\end{array}$ & 2007 & success & explicit \\
\hline 1025 & $\begin{array}{l}\text { Enlisting of external contrac- } \\
\text { tors }\end{array}$ & 3 & 8 & 3 & 12 & 3 & 12 & PM & $\begin{array}{l}\text { Fowler } \\
\text { and Horan }\end{array}$ & 2007 & success & explicit \\
\hline 1026 & $\begin{array}{l}\text { Lack of adequate user involve- } \\
\text { ment }\end{array}$ & 3 & 8 & 3 & 8 & 3 & 8 & PM & $\begin{array}{l}\text { Fowler } \\
\text { and Horan }\end{array}$ & 2007 & failure & explicit \\
\hline 1027 & $\begin{array}{l}\text { Lack of top-management com- } \\
\text { mitment to the project }\end{array}$ & 3 & 15 & 3 & 15 & 3 & 15 & PM & $\begin{array}{l}\text { Fowler } \\
\text { and Horan }\end{array}$ & 2007 & failure & explicit \\
\hline 1028 & Poor/inadequate user training & 3 & 11 & 3 & 11 & 3 & 8 & PM & $\begin{array}{l}\text { Fowler } \\
\text { and Horan }\end{array}$ & 2007 & failure & explicit \\
\hline 1029 & $\begin{array}{l}\text { Lack of cooperation from us- } \\
\text { ers (user resistance) }\end{array}$ & 3 & 9 & 3 & 9 & 3 & 8 & PM & $\begin{array}{l}\text { Fowler } \\
\text { and Horan }\end{array}$ & 2007 & failure & explicit \\
\hline 1030 & $\begin{array}{l}\text { Perceived usefulness of tech- } \\
\text { nology }\end{array}$ & 4 & 27 & 4 & 27 & 4 & 27 & BPI & Rodriguez et al. & $2020 b$ & success & explicit \\
\hline 1031 & Kind of business model & 2 & 24 & 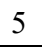 & 24 & 5 & 24 & BPI & Rodriguez et al. & $2020 \mathrm{~b}$ & success & explicit \\
\hline 1032 & Technology adoption & 7 & 29 & 7 & 29 & 7 & 29 & $\mathrm{BPR}$ & Rodriguez et al. & $2020 \mathrm{a}$ & success & explicit \\
\hline 1033 & Organizational adaptation & 6 & 26 & 6 & 26 & 6 & 26 & $\mathrm{BPR}$ & Rodriguez et al. & $2020 \mathrm{a}$ & success & explicit \\
\hline 1034 & Organizational resistance & 6 & 25 & 6 & 25 & 6 & 25 & $\mathrm{BPR}$ & Rodriguez et al. & $2020 \mathrm{a}$ & success & explicit \\
\hline
\end{tabular}




\section{Appendix C: Expert Interviews Overview}

\begin{tabular}{|c|c|c|c|c|c|c|}
\hline Company & PDP & Expert & Operating region & Position & $\begin{array}{l}\text { Functional/ } \\
\text { technical }\end{array}$ & Experience \\
\hline \multirow{7}{*}{$\begin{array}{l}\text { Company } \mathrm{P} \\
\text { Turnover: } \\
\text { 3,5 Billion } \\
\text { Employees: } \\
>20.000 \\
\text { Production sites: }>100\end{array}$} & \multirow{4}{*}{$\begin{array}{l}\text { P1: } \\
\text { Digital flow } \\
\text { production }\end{array}$} & E1 & Germany & Project Sponsor & both & 31 years \\
\hline & & E2 & Portugal & Employee & technical & 10 years \\
\hline & & E3 & Germany & Project Manager & technical & 6 years \\
\hline & & E4 & Germany & Employee & technical & 8 years \\
\hline & \multirow{3}{*}{$\begin{array}{l}\text { P2: } \\
\text { IoT platform }\end{array}$} & E5 & South Africa & Project Manager & functional & 17 years \\
\hline & & E6 & South Africa & Employee & functional & 12 years \\
\hline & & E7 & Germany & Project Manager & technical & 11 years \\
\hline \multirow{4}{*}{$\begin{array}{l}\text { Company A } \\
\text { Turnover: } \\
6.5 \text { Billion } € \\
\text { Employees: } \\
>20.000 \\
\text { Production sites: }>100\end{array}$} & \multirow{4}{*}{$\begin{array}{l}\text { A1: } \\
\text { Industrial IoT } \\
\text { application }\end{array}$} & E8 & England & Employee & functional & 7 years \\
\hline & & \begin{tabular}{|l|} 
E9 \\
\end{tabular} & England & Project Manager & functional & 13 years \\
\hline & & E10 & Germany & Project Manager & technical & 14 years \\
\hline & & E11 & Germany & Project Sponsor & both & 30 years \\
\hline \multirow{7}{*}{$\begin{array}{l}\text { Company } \mathrm{H} \\
\text { Turnover: } \\
\text { 170 Million } € \\
\text { Employees: } \\
>1.000 \\
\text { Production sites: } 5-10\end{array}$} & \multirow{2}{*}{$\begin{array}{l}\text { H1: } \\
\text { IoT platform }\end{array}$} & E12 & Germany & Project Manager & functional & 32 years \\
\hline & & E13 & Germany & $\begin{array}{l}\text { Project Sponsor } \\
(\mathrm{CIO})\end{array}$ & both & 33 years \\
\hline & \multirow{3}{*}{$\begin{array}{l}\mathrm{H} 2 \text { : } \\
\text { Predictive } \\
\text { maintenance }\end{array}$} & E14 & Germany & Project Manager & technical & 17 years \\
\hline & & E15 & Germany & Project Manager & functional & 6 years \\
\hline & & E16 & Germany & Employee & technical & 12 years \\
\hline & \multirow{2}{*}{$\begin{array}{l}\text { H3: } \\
\text { Big data analyt- } \\
\text { ics }\end{array}$} & E17 & Germany & Employee & functional & 14 years \\
\hline & & E18 & Germany & Project Manager & technical & 4 years \\
\hline \multirow{3}{*}{$\begin{array}{l}\text { Company T } \\
\text { Turnover: } \\
290 \text { Million } € \\
\text { Employees: } \\
\text { >700 } \\
\text { Production sites: } 5-10\end{array}$} & \multirow{3}{*}{$\begin{array}{l}\text { T1: } \\
\text { Robotic process } \\
\text { automation }\end{array}$} & \begin{tabular}{|l|} 
E19 \\
\end{tabular} & Germany & Employee & technical & 8 years \\
\hline & & \begin{tabular}{|l|} 
E20 \\
\end{tabular} & Germany & Project Manager & technical & 21 years \\
\hline & & E21 & Germany & $\begin{array}{l}\text { Project Sponsor } \\
(\mathrm{CFO})\end{array}$ & both & 23 years \\
\hline
\end{tabular}




\section{Appendix D: Detailed Context-Description of the investigated PDPs}

PDP P1 related to the implementation of digital flow production. In P1, company P was attempting to replace the manual material logistics, which implied that, until that point, employees had manually planned and operated transportation processes. This PDP aimed at enabling autonomous material logistics and at adapting these to current production requirements. By doing so, company $\mathrm{P}$ aimed to improve logistics processes and further increase their effectiveness via a digital end-to-end analysis of the entire production process. In PDP P2, company P introduced an IoT platform for its production processes and products. In this PDP, the company aimed to replace the production management process. Aside from the MES and ERP, the systems of the production plants were not integrated with other systems or devices. To identify products, employees labeled the product components and manually traced them with barcode scanners. P2 established a connecting layer between machines, which enabled product tracing. The production plant reached an unprecedented level of connectivity which enabled the rapid exchange of information and allowed each network participant to control machines remotely. Based on P2, company $\mathrm{P}$ introduced big data analytics.

PDP A1 refers to the introduction of the Industrial IoT. A1 involves manual quality control processes being replaced with automated quality control measures based on sensor data. The manual process asked employees to measure the quality of goods produced as well as the key figures of machines used. The objective of the PDP was to reduce the number of manual processes needed to manage the quality of products and machines. Furthermore, company A equipped product components with sensors with which employees could track and trace products throughout the production process.

PDP H1 describes the introduction of an IoT platform connecting smart devices, the manufacturing enterprise system (MES), and enterprise resource planning (ERP). Formerly, each equipment manufacturer provided proprietary software and interfaces. During H1, company H re-engineered the process of production control. Previously, the MES did not meet its requirements, and an interface between the MES and the ERP did not exist. Company H thus aimed to unify the interfaces of the plant and machine suppliers, homogenize data sources, and decentralize control entities. The IoT platform supports the coordination and homogenization of the data collection. Overall, the machines of three plants were connected during the project. PDP H2 describes the introduction of a predictive maintenance solution. In $\mathrm{H} 2$, the project team aimed to redesign the process of plant maintenance. Before $\mathrm{H} 2$, employees and sensors collected data in an unsystematic manner. Real-time production data analysis did not exist. Employees had to maintain machines at various, imprecise time intervals, which often led to unnecessary 
maintenance being carried out. The new data analytics introduced during $\mathrm{H} 2$ enabled the use of multiple, formerly unused production data. Company $\mathrm{H}$ was, thus, able to design a framework to homogenize process data, synchronized data collectors, and installed new data collection points. PDP H3 refers to the introduction of big data analytics of energy generation and consumption data. Company $\mathrm{H}$ employed $\mathrm{H} 3$ to establish a process for energy management. In practical terms, H3 was conducted to enable standardized data collection, homogenize data collected, and set-up data analytics. Thereby, company H expected to identify opportunities to save energy by improving the energy efficiency of production. Prior to H3, data collection and analysis were in their infancy and the analysis of energy data had not been possible. Employees could not make qualitative statements about energy generation or use. The PDP is designed to facilitate the exploration of knowledge about energy consumption and, thus, enable improved energy management. Company H now expects additional sales opportunities to arise via the monetarization of knowledge about their energy demand, as energy providers need access to such information for their demand management.

Finally, PDP T1 describes the introduction of robotic process automation in the sales department. Company T aimed to replace the order release process with T1. Formerly, the options, once orders were received, were manual acceptance, acceptance despite discrepancies, or rejection. Employees, thereby, manually caught up authorized signatures, which is why the schedule of the sales representative was a bottleneck that regularly led to stand-time. With this PDP, company $\mathrm{T}$ sets out to first record the acceptance of orders and examine the cash audit of the outstanding accounts of the ordering organization. Second, the administrative department routed corresponding information to the sales representative in charge. Three, the resumption of the order and its release or rejection by the sales representative, fourth, the terminal of the production plant had to be prepared to release or reject the order consequently. 


\section{Appendix E: Interview Process}

During the interviews, the interviewees were encouraged to speak freely and at length, allowing us to offset researcher bias, to gain practical knowledge (Heidt et al., 2019), and to allow for the identification of novel factors (Myers and Newman, 2007). As we sought to gain a deep understanding of the selected PDPs, the interviews were led actively sharing relevant knowledge and discussing candidate SFs (Myers and Newman, 2007). Throughout the interaction with the interviewees, we used gained insights to validate and refine the ex-ante list of candidate SFs. Based on the interview protocols (Sarker et al., 2013), each interview followed the same structure (Greener, 2008). All interviews lasted 60 to 90 minutes and were recorded. We transcribed the recordings and coded the data via qualitative content analysis (Krippendorff, 2013) supported by MAXQDA. To offset potential bias, interviewees and companies were anonymized (Benbasat et al., 1987). 


\section{Appendix F: Mapping of the Success Codes and SF}

\begin{tabular}{|c|c|}
\hline SF & No. of the success code: shortened code [reference]; \\
\hline Goal Clarity & $\begin{array}{l}\text { - No. 211: Clear objective (Pankratz and Loebbecke, 2011); } \\
\text { - No. 338: Clear objective and scope (Jugdev and Müller, 2005); } \\
\text { - No. 726: Develop and communicate clear written mission and vision statements (Guimaraes, } \\
\text { 1997) }\end{array}$ \\
\hline $\begin{array}{l}\text { Strategy } \\
\text { Integration }\end{array}$ & $\begin{array}{l}\text { - No. 77: Organizational alignment towards digital (Holotuik and Beimborn, 2017); } \\
\text { - No. 127: No linkage with to overall business goals and objectives (Siha and Saad, 2008); } \\
\text { - No. 729: Adopt an integrated approach to IT and business planning (Guimaraes, 1997) }\end{array}$ \\
\hline $\begin{array}{l}\text { Infrastructural } \\
\text { Agility }\end{array}$ & $\begin{array}{l}\text { - No. 88: Modular IT platform (Holotuik and Beimborn, 2017); } \\
\text { - No. 352: Standard software architecture (Jugdev and Müller, 2005); } \\
\text { - No. 547: Infrastructure in place to support the developers (Avital, 2003) }\end{array}$ \\
\hline $\begin{array}{l}\text { Organizational } \\
\text { Agility }\end{array}$ & $\begin{array}{l}\text { - No. 81: Multi-level and multi-speed organization for faster reaction (Holotuik and Beimborn, } \\
\text { 2017); } \\
\text { - No. 351: Organizational adaptability (Jugdev and Müller, 2005); } \\
\text { - No. 967: Organizational structure (Shenhar et al., 2002) }\end{array}$ \\
\hline Employee Agility & $\begin{array}{l}\text { - No. 483: Organizational resistance (Al-Mashari and Zairi, 1999); } \\
\text { - No. 584: User resistance (Umble et al., 2003); } \\
\text { - No. 602: Skepticism about project result (Abdolvand et al., 2008) }\end{array}$ \\
\hline $\begin{array}{l}\text { Innovation } \\
\text { Attitude }\end{array}$ & $\begin{array}{l}\text { - No. 356: Encouraging new ideas (Dvir et al., 1998); } \\
\text { - No. 555: Introduce new products (Malinova et al., 2014); } \\
\text { - No. 917: Openness to change (Paper and Chang, 2005) }\end{array}$ \\
\hline $\begin{array}{l}\text { Management } \\
\text { Agility }\end{array}$ & $\begin{array}{l}\text { - No. 298: Agile requirements (Jugdev and Müller, 2005); } \\
\text { - No. 357: Willingness to consider changes and new approaches (Dvir et al., 1998); } \\
\text { - No. 554: Adapt to external changes (Malinova et al., 2014) }\end{array}$ \\
\hline $\begin{array}{l}\text { Resource } \\
\text { Agility }\end{array}$ & $\begin{array}{l}\text { - No. 48: Agility to reallocate resources (Holotuik and Beimborn, 2017) } \\
\text { - No. 997: Meeting the budget (Besteiro et al., 2015); } \\
\text { - No. 1016: Resource availability (Gomes and Romão, 2016) }\end{array}$ \\
\hline Risk Attitude & $\begin{array}{l}\text { - No. 52: Accept failure (Holotuik and Beimborn, 2017); } \\
\text { - No. 143: Organization's processes for assigning ownership of risks (Costantino et al., 2015); } \\
\text { - No. 284: Company-wide education on the concepts of risk management (Cooke-Davies, 2002) }\end{array}$ \\
\hline $\begin{array}{l}\text { Customer } \\
\text { Knowledge }\end{array}$ & $\begin{array}{l}\text { - No. 70: Outstanding customer experience and satisfaction (Holotuik and Beimborn, 2017); } \\
\text { - No. 524: Being recognized as valuable by users (Avital, 2003); } \\
\text { - No. 1013: Market Impact (Gomes and Romão, 2016) }\end{array}$ \\
\hline $\begin{array}{l}\text { Employee } \\
\text { Domain } \\
\text { Knowledge }\end{array}$ & $\begin{array}{l}\text { - No. 163: Team member's skills (Pankratz and Loebbecke, 2011); } \\
\text { - No. 620: Business skills of the project team (Dezdar and Ainin, 2011); } \\
\text { - No. 1019: Project personnel knowledge/ skills (Fowler and Horan, 2007) }\end{array}$ \\
\hline $\begin{array}{l}\text { Employee Tech- } \\
\text { nology } \\
\text { Knowledge }\end{array}$ & $\begin{array}{l}\text { - No. 450: High technical level (Dvir et al., 1998); } \\
\text { - No. 624: Technical skills of the project team (Dezdar and Ainin, 2011); } \\
\text { - No. 951: IT knowledge (Paper and Chang, 2005) }\end{array}$ \\
\hline $\begin{array}{l}\text { Management Do- } \\
\text { main Knowledge }\end{array}$ & $\begin{array}{l}\text { - No. 22: Project manager's leadership style (Irvine and Hall, 2015); } \\
\text { - No. 63: Capability to develop new business models (Holotuik and Beimborn, 2017); } \\
\text { - No. 252: Characteristics of the project team leader (Ika, 2009) }\end{array}$ \\
\hline $\begin{array}{l}\text { Management } \\
\text { Technology } \\
\text { Knowledge }\end{array}$ & $\begin{array}{l}\text { - No. 448: Technical issues managed (Dvir et al., 1998); } \\
\text { - No. 451: A technical leader (Dvir et al., 1998); } \\
\text { - No. 828: Technical leadership skills (Hughes } \text { et al., 2017) }\end{array}$ \\
\hline $\begin{array}{l}\text { Process Improve- } \\
\text { ment Skills }\end{array}$ & $\begin{array}{l}\text { - No. 105: Process improvement (Siha and Saad, 2008); } \\
\text { - No. 235: Improve the underlying process, where necessary, prior to digitizing the process (Abol- } \\
\text { lado et al., 2017); } \\
\text { - No. 461: Effective process redesign (Al-Mashari and Zairi, 1999) }\end{array}$ \\
\hline $\begin{array}{l}\text { Process } \\
\text { Knowledge }\end{array}$ & $\begin{array}{l}\text { - No. 109: Questioning the fundamental assumptions of a process (Siha and Saad, 2008); } \\
\text { - No. 237: Focus first on processes that are fully understood (Abollado et al., 2017); } \\
\text { - No. 563: Identify \& understand weaknesses of your processes (Malinova et al., 2014) }\end{array}$ \\
\hline
\end{tabular}




\begin{tabular}{|c|c|}
\hline $\begin{array}{l}\text { Process } \\
\text { Design }\end{array}$ & $\begin{array}{l}\text { - No. 68: Data-driven and digitally automated process (Holotuik and Beimborn, 2017); } \\
\text { - No. 103: Failure to define a beginning and end to the process (Siha and Saad, 2008); } \\
\text { - No. 956: Design techniques (Shenhar et al., 2002) }\end{array}$ \\
\hline $\begin{array}{l}\text { Process } \\
\text { Monitoring }\end{array}$ & $\begin{array}{l}\text { - No. 98: Focus on the measures of process success (Siha and Saad, 2008); } \\
\text { - No. 174: Control of the development process (Pankratz and Loebbecke, 2011); } \\
\text { - No. 231: Workflow progress tracking tool (Abollado et al., 2017); }\end{array}$ \\
\hline $\begin{array}{l}\text { Customer } \\
\text { Integration }\end{array}$ & $\begin{array}{l}\text { - No. 72: Direct contact for customer-centricity (Holotuik and Beimborn, 2017); } \\
\text { - No. 411: Team includes end-user representatives (Dvir } \text { et al., 1998); } \\
\text { - No. 1026: Lack of adequate user involvement (Fowler and Horan, 2007) }\end{array}$ \\
\hline $\begin{array}{l}\text { Employee } \\
\text { Support }\end{array}$ & $\begin{array}{l}\text { - No. 483: Organizational resistance (Al-Mashari and Zairi, 1999); } \\
\text { - No. 584: User resistance (Umble et al., 2003); } \\
\text { - No. 602: Skepticism about project result (Abdolvand et al., 2008) }\end{array}$ \\
\hline $\begin{array}{l}\text { External Commu- } \\
\text { nication }\end{array}$ & $\begin{array}{l}\text { - No. 420: Communication with all subcontractors (Dvir } \text { et al., 1998); } \\
\text { - No. 479: Effective use of consultants (Al-Mashari and Zairi, 1999); } \\
\text { - No. 891: Strong communication outwards (Ram et al., 2013) }\end{array}$ \\
\hline $\begin{array}{l}\text { Internal Commu- } \\
\text { nication }\end{array}$ & $\begin{array}{l}\text { - No. 262: Ineffective communication behaviors (Kirsch and Slaughter, 2013); } \\
\text { - No. 631: Development of a communication plan (Antony et al., 2012); } \\
\text { - No. 744: Communication throughout the project (Clarke, 1999) }\end{array}$ \\
\hline $\begin{array}{l}\text { Partner } \\
\text { Integration }\end{array}$ & $\begin{array}{l}\text { - No. 116: Sharing forums among comparative firms (Siha and Saad, 2008); } \\
\text { - No. 859: Partnership with vendor (Remus, 2007); } \\
\text { - No. 900: integration with partner organizations' information systems (Ram et al., 2013) }\end{array}$ \\
\hline $\begin{array}{l}\text { Project } \\
\text { Monitoring }\end{array}$ & $\begin{array}{l}\text { - No. 166: Performance measurement systems (Pankratz and Loebbecke, 2011); } \\
\text { - No. 290: Provide direct feedback on current project performance (Cooke-Davies, 2002); } \\
\text { - No. 506: Measurement of costs and benefits (Frey and Buxmann, 2012) }\end{array}$ \\
\hline $\begin{array}{l}\text { Project } \\
\text { Preparation }\end{array}$ & $\begin{array}{l}\text { - No. 25: Quality of planning (Irvine and Hall, 2015); } \\
\text { - No. 255: Poorly understood or ill-structured project requirements (Kirsch and Slaughter, 2013); } \\
\text { - No. 990: Planning the project (Besteiro et al., 2015) }\end{array}$ \\
\hline $\begin{array}{l}\text { Team } \\
\text { Portfolio }\end{array}$ & $\begin{array}{l}\text { - No. 181: Right mix of team members (Pankratz and Loebbecke, 2011); } \\
\text { - No. 577: A great implementation team (Umble et al., 2003); } \\
\text { - No. 881: Teamwork and project team composition, competence, and compensation (Ram et al., } \\
\text { 2013) }\end{array}$ \\
\hline $\begin{array}{l}\text { Team } \\
\text { Support }\end{array}$ & $\begin{array}{l}\text { - No. 202: Team members' motivation (Pankratz and Loebbecke, 2011); } \\
\text { - No. 472: Empowerment of both individuals and teams (Al-Mashari and Zairi, 1999); } \\
\text { - No. 1015: Team Engagement (Gomes and Romão, 2016) }\end{array}$ \\
\hline $\begin{array}{l}\text { Top } \\
\text { Management Sup- } \\
\text { port }\end{array}$ & $\begin{array}{l}\text { - No. 152: Top management support (Costantino et al., 2015); } \\
\text { - No. 555: Commitment by top management (Umble et al., 2003); } \\
\text { - No. 725: Motivated by chief executive willing to be held accountable for project success } \\
\text { (Guimaraes, 1997) }\end{array}$ \\
\hline $\begin{array}{l}\text { Technology Com- } \\
\text { plexity }\end{array}$ & $\begin{array}{l}\text { - No. 218: Technology characteristics (Pankratz and Loebbecke, 2011); } \\
\text { - No. 444: Technical feasibility checked (Dvir et al., 1998); } \\
\text { - No. 826: feasibility undertaken (Hughes et al., 2017) }\end{array}$ \\
\hline $\begin{array}{l}\text { Technology } \\
\text { Maturity }\end{array}$ & $\begin{array}{l}\text { - No. 47: Project deliverable/ technology - maturity (Irvine and Hall, 2015); } \\
\text { - No. 267: Maturity of software (Kirsch and Slaughter, 2013); } \\
\text { - No. 552: Knowledge of the technology in use (Avital, 2003) }\end{array}$ \\
\hline
\end{tabular}




\section{Appendix G: Identification of New Candidate Success Factors}

Overall, we identified seven candidate SFs not included in the literature. Three underline the importance of partners as carriers of expertise within PDPs. Our interviewees consistently provided two examples referring to the importance of partners for PDP success. Either partners' domain knowledge links to the process to be digitalized (Yayavaram et al., 2018) or partners hold essential technological expertise regarding DTs (Flor et al., 2018) to be used in the PDP. A project manager of PDP P2 stated: "Partners become ever more important. Digitalization is none of our core competencies, which is why we must trust and rely on strong partners" (project manager P2). Beyond partner domain knowledge and partner technology knowledge, the interviewees stressed the importance of partner agility. They emphasized the importance of partner agility in the event of changes during PDPs, as partners then must deliver new input, show clemency in renegotiations, or react quickly (Ren et al., 2005). The $\mathrm{CIO}$ of company $\mathrm{H}$, for example, claimed that "it becomes ever more important that partners can quickly react to new circumstances, for example, when we receive new requirements from our own customers" (CIO H).

Another four new candidate SFs fall into the categories of strategy, culture, people, and technology. In the strategy category, we found digital ambition as a candidate SF, which we define as the continuous focus on the digitalization of the organization and its business processes (Gartner, 2017). This factor involves an active interest and support in taking advantage of DTs, especially their exposure through the management. In PDP T1, there never was a lack of passive support from the top management, as the project sponsor had much confidence in the PDP team. However, this very sponsor was not interested in the implementation of the chosen DT, as the following statement highlights: "The guys from IT actually know what they do. I don't have to care about them, and I won't do as I'm not interested in those technologies [...]" (project sponsor T1). This lack of active support reduced the team members' motivation. The negative effect of missing digital ambition became particularly evident when the management opportunistically assigned team members to other projects. In the original project, the lack of sponsor interest also affected the motivation of employees not being part of the PDP core team, who regularly canceled meetings without justification. However, this sort of behavior was not apparent in projects championed by the project sponsor. By contrast, in company H, digital ambition strengthened the motivation of PDP H1's team members. The CIO, who even had received awards for fully embracing digitalization, continuously communicated a cheerful outlook toward the company's PDPs. Accordingly, the employees felt highly motivated by the recognition they received from the project sponsor and manager. Their awareness of this positive feedback resulted in increased output and commitment, which in turn helped ensure PDP H1's effectiveness and efficiency. 
In the culture category, we found that organizations must foster a digitalization attitude, which we define as the PDP members' willingness-to-change and open-mindedness toward DTs (Koleva, 2019). PDP P2, for example, demonstrated the need for digitalization attitude as a part of the corporate culture. As the PDP team missed to involve prospective process participants during the implementation, the management introduced new plant strategies including the cultivation of a positive digitalization attitude. Over time, employees showed increased openmindedness toward the introduced Internet of Things platform, as becomes evident in a project manager's statement: "The employees did not even realize what happens with such technologies. [...] the publishing of the plant vision for digitalization brought awareness to the ordinary employees, who normally do their job without further reflection." (project manager $\mathrm{P} 2)$.

In the people category, we found data analysis as a candidate SF, which previous studies commonly defined as the central, decentral, or hybrid usage of analytical and decision-making capabilities for diagnostic, descriptive, prescriptive, and predictive purposes (Porter and Heppelmann, 2015). Today, digitalization increases the application possibilities of analytics (Clarke, 2016). In each investigated PDP, the team members used data analysis methods. Even in those PDPs that did not rely on data analysis, the team members said that data analysis was becoming increasingly important. The positive influence on PDP success could be observed in, for example, projects H2 and $\mathrm{H} 3$, both of which involve analytical approaches and, as the sponsors and managers underlined, consequently rely on data analysis. Team members of $\mathrm{H} 2$ and $\mathrm{H} 3$ classified data analysis as the factor with the greatest impact on PDP success. The technical project manager of $\mathrm{H} 3$ stated that they "[...] just can proceed with knowledge in analytics: the more knowledge, the faster and safer the success" (technical project manager H3). However, a lack of data analysis knowledge may even cause problems in PDPs that do not use DTs for data analysis. In PDPs implementing such DTs, disregard for data analysis jeopardizes the whole project. Consequently, we highlight the positive effect that data analysis has on PDP success.

In the technology category, we found a positive effect of technology comprehensibility on PDP success i.e., the level of abstractness of the DT used in a PDP (Flor et al., 2018). In fact, this candidate SF focuses on the maturity and complexity of a DT, which, if mis-assessed, can negatively impact PDP success. When implementing DTs, it is the team members' understanding of functionality and impact that poses a challenge. For example, the CIO of company H stated that "with the speed of DTs emerging, how can we ensure the knowledge and skillset needed? [...] We cannot update the knowledge about technology as quickly as those digital technologies emerge. The only control we have is to select DTs which are understandable for our employees and the management” (CIO H). 


\section{Appendix H: Refinement of Existing Candidate Success Factors}

In addition to the identification of new candidate SFs, we could also refine two existing factors based on our findings from the interviews. First, we had to split goal clarity into the candidate SFs process goal clarity and project goal clarity. We define process goal clarity as the transparency and consistency of the goals of the business process affected by the PDP (Peralta et al., 2015). By contrast, project goal clarity refers to the transparency and consistency of the goals of the PDP itself (Raziq et al., 2018). The clarity of PDP goals and their communication are supposed to positively affect PDP success, a relationship which we observed in PDP H3. The impact of project goal clarity became evident as company $\mathrm{H}$ concretized the process affected by the PDP. Previously, company $\mathrm{H}$ had planned the PDP as a big data analytics project without specifying the underlying process. The project sponsor, manager, and employees consistently observed that the project sped up after the participants had identified the process affected. Accordingly, we included process and project goal clarity. The CIO of company $\mathrm{H}$, for example, emphasizes that H1 "runs like clockwork because the project's scope is clearly defined".

Moreover, we changed the SF candidate infrastructural agility to infrastructural readiness, defined as the extendibility, compatibility, and robustness of the organization's technological infrastructure (Haddad et al., 2018). During the interviews, PDP team members frequently stated how important it is that infrastructure does not hinder the implementation of DTs. In the PDPs P1 and A1, the project teams had to overcome several infrastructural challenges. In P1, the PDP team had to renew the technological infrastructure that provided the foundation for the PDP. The fact that the planning employees were not involved in the PDP core team led them to underestimate the effort of this step. In A1, some members of the project team first had to implement the infrastructure, including the purchase of technical components. The actual PDP then began with reduced resources as the time and effort the employees had already invested did not serve the needs of the PDP. The importance of infrastructural readiness becomes evident in a project sponsor's (A1) statement: "When infrastructure is not ready to implement the PDP's technology, the PDP is doomed". 